ARGONNE NATIONAL LABORATORY

9700 South Cass Avenue

Argonne, Illinois 60439

\title{
ANL/APS/TB-45
}

\section{Undulator A Magnetic Properties and Spectral Performance}

\author{
by Roger J. Dejus, Isaac B. Vasserman, Shigemi Sasaki, \\ and Elizabeth R. Moog \\ Experimental Facilities Division \\ Advanced Photon Source
}

May 2002

work sponsored by

U.S. DEPARTMENT OF ENERGY

Office of Science 


\section{Table Of Contents}

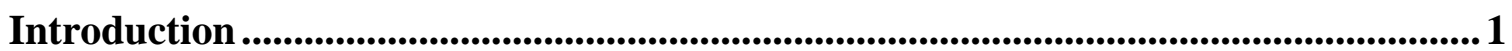

First Beam and Spectral Measurements ...............................................................................2

Magnetic Specifications and Magnetic Measurements...................................................2

Spectral Performance ................................................................................................................................

On-Axis Brilliance Tuning Curves ....................................................................... 7

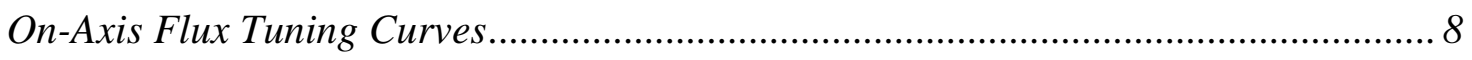

Canted and Tandem IDs Compared with Undulator A................................................ 9

On-Axis Brilliance Tuning Curves for Canted and Tandem IDs................................ 9

On-Axis Flux Tuning Curves for Canted and Tandem IDs ...................................... 11

Power Considerations .............................................................................................................................. 12

Angular Distribution and Effect of Emittance.......................................................... 13

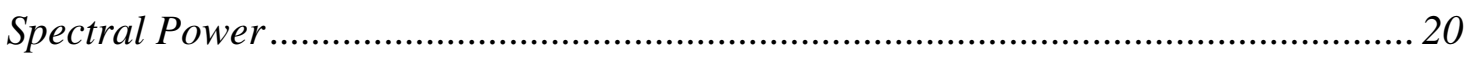

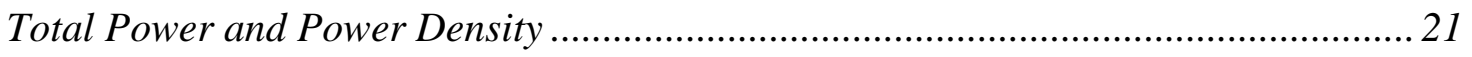

Prospect for Increased Brilliance.....................................................................................................23

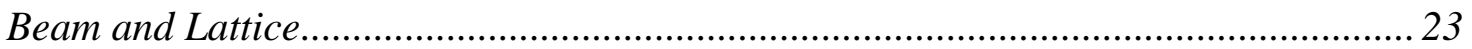

Insertion Device Changes ................................................................................ 23

Acknowledgments..............................................................................................................................24

Appendix A: The APS Storage Ring …...................................................................................25

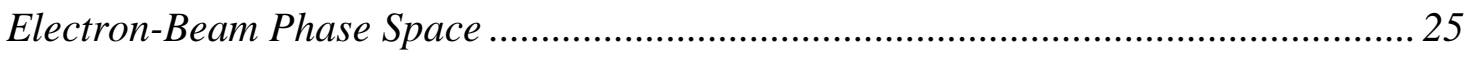

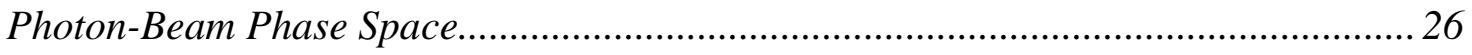

Brilliance Estimate from the Flux in the Central Cone ……...................................... 27

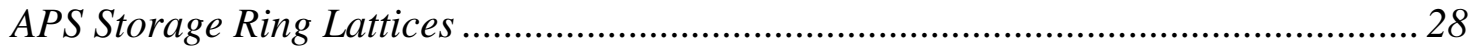

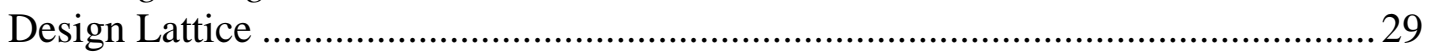

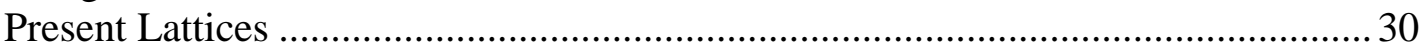

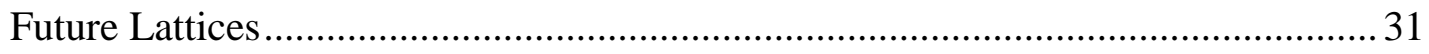

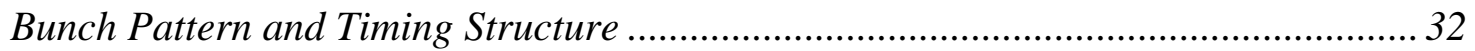


Appendix B: Additional Undulator A Magnetic Properties ..............................................34

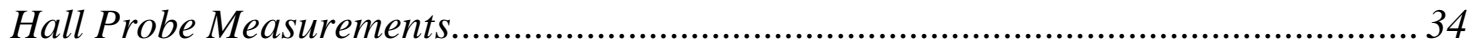

Coil Measurements.............................................................................................. 40

Appendix C: Undulator 2.7 cm Magnetic Properties ....................................................... 44

Appendix D: On-Axis Brilliance for APS Bending Magnet, Wiggler, and Other IDs

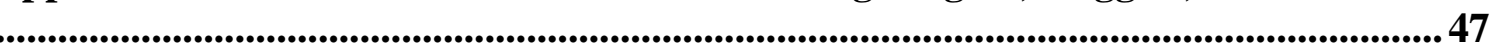

References …..................................................................................................................................... 48 


\section{Introduction}

The Undulator A at the Advanced Photon Source (APS) is a planar permanent magnet hybrid device optimized for generating $\mathrm{x}$-rays from $3 \mathrm{keV}$ to $45 \mathrm{keV}$ by using the first, third, and fifth radiation harmonics. It also produces $\mathrm{x}$-rays above this energy. For high-energy experiments, users are relying on using higher harmonics, which has become possible because of improved undulator technology over the past decade. The Undulator A has been designed to provide continuous energy coverage with no significant drop in brilliance when switching between the harmonics, i.e., the tuning curve from one harmonic to the next intersect.

The undulator has a period length of $3.30 \mathrm{~cm}$ and has 72 magnetic periods (144 poles) for a total length of $2.4 \mathrm{~m}$. The undulator was initially described in Technical Bulletin ANL/APS/TB-3 (1993) [1] and subsequently in ANL/APS/TB-17 (1994) [2]. Both documents were published before the first undulator had been delivered to the APS so that the information given was based on design specifications. A three-dimensional (3D) magnetic modeling code was used to estimate the magnetic field vs. gap, and computer simulations were used to predict the on-axis brilliance, flux, and power for the APS design lattice using an ideal undulator magnetic field, i.e., pure sinusoidal variation of the magnetic field along the undulator. The magnetic field strength given in earlier publications was what was required by the undulator purchase contract.

Since then, 23 Undulator A devices have been measured, tuned, and installed in the storage ring. It should be noted that undulators are removed periodically from the storage ring for retuning, and the values listed in this document are therefore subject to change. This document focuses on the measured magnetic properties and the spectral performance of these devices. We will show the calculated on-axis brilliance and flux for the present APS lattice ("low-emittance" lattice with 1.0\% coupling) and compare those with the APS design lattice. The radiated power and on-axis power density will also be given. We will also look into the future for prospects to obtain even higher brilliances and fluxes by further improvements to the lattice and by using longer undulators with shorter period lengths. (We provide four appendices with additional information on A) the APS storage ring, B) Undulator A measured magnetic properties, and C) one shorterperiod device. In Appendix D, we also compare the Undulator A on-axis brilliance vs. other sources at the APS for the present low-emittance operation.)

All spectral and power calculations in this document were performed with computer codes from the XOP suite of X-ray optics programs that assume an ideal undulator magnetic field [3]. We have also been able to accurately predict the reduction in brilliance of higher harmonics due to magnetic field errors by using the code UR that uses a measured magnetic field as input [4], and specific references are given in the text where such experience was used. Although a variety of properties of general interest have been addressed here, specific needs by individual users will be met by contacting the authors for more information. 


\section{First Beam and Spectral Measurements}

The first undulator beam at the APS was observed on August 9, 1995 [5], and a summary of the first results was given by Cai et al. [6]. Based on the characterization of the device that included magnetic measurements, absolute spectral flux measurements with a gas-scattering spectrometer, and crystal spectrometer measurements of the spectrum (up to the $17^{\text {th }}$ harmonic), it was concluded that the undulator had exceeded the demanding magnetic requirements that were specified in the design.

In 1996, experimental work was continued on the same device, and results were published that compared the measured absolute flux with calculations from XOP [3] and the code UR [4]. Very good agreement was found; the small discrepancy could be attributed to magnetic field errors and uncertainties in the beam parameters [7].

In 1997, the Undulator A type device was characterized at even higher photon energies $(50-200 \mathrm{keV})$ by Shastri et al. [8]. Again, they found that the measured spectra agreed with predictions from computer calculations, which included the measured magnetic fields, as well as a realistic beam emittance and beam energy spread [4]. A very high harmonic content was accurately reproduced above $80 \mathrm{keV}$ confirming the high magnetic quality of the insertion device (and the accurate knowledge of the beam parameters at the time).

A second device was diagnosed by Ilinski later in 1997 that included simultaneous measurements of the beam parameters from detuned harmonic energies and the absolute flux using a crystal spectrometer setup [9]. He found that the measured absolute flux was within one percent of the calculated flux for the third harmonic (for the fifth harmonic, the measured flux was $15 \%$ less than calculated). The reduction in intensity for the third harmonic due to magnetic field errors could be estimated to be less than $10 \%$, again confirming the high magnetic quality of the undulator.

\section{Magnetic Specifications and Magnetic Measurements}

All insertion devices (IDs) undergo rigorous magnetic tuning before they are installed in the storage ring. Stringent magnetic criteria must be met at all undulator gaps so as to not perturb the stored beam in an undesirable manner. In addition to shimming for low distortion of the beam (as expressed in tolerances for the first and second field integrals and higher multipole moments), the undulators need to be shimmed for high spectral brilliance [10]. To obtain high spectral brilliance, the rms phase error for the devices must be kept small. The original tolerance requirement was that the rms phase error be less than $8^{\circ}$ to ensure that the brilliance of the third harmonic would be at least $70 \%$ of the ideal for a zero-emittance beam. This was a new design parameter for the vendor. Traditionally, the rms peak magnetic field error had been used. However, the rms phase error specification is more important because the intensity degradation of the harmonics for a zero-emittance beam for well-optimized devices depends approximately 
exponentially on this parameter, $\frac{I_{n}}{I_{n 0}} \sim \exp \left(-n^{2} \sigma_{\phi}^{2}\right)$, where $n$ is the harmonic number and $\sigma_{\phi}$ is the rms phase error [11]. (In reality, when the beam emittance and the angular acceptance of the photon beam are taken into account, the sensitivity to the phase errors is less.) It should also be noted that intensity of the first harmonic is not particularly sensitive to phase errors. The rms phase error and other important parameters that were specified and calculated for Undulator A are given in Table 1. We have chosen to list the average values that were obtained from the magnetic measurements of 23 IDs with rms variations, where applicable.

Table 1: Undulator A parameters, specifications and measured values.

\begin{tabular}{|c|c|c|c|}
\hline Parameter & \multicolumn{2}{|c|}{ Specified Value } & Measured Value $^{1)}$ \\
\hline Magnet material & \multicolumn{3}{|l|}{$\mathrm{Nd}-\mathrm{Fe}-\mathrm{B}$} \\
\hline Pole material & \multicolumn{3}{|c|}{ Vanadium permendur } \\
\hline Period length, $\lambda_{u}$ & \multicolumn{3}{|c|}{$3.30 \mathrm{~cm}$} \\
\hline Number of periods, $N$ & 72 & 2) & \\
\hline Length, $L$ & $2.4 \mathrm{~m}$ & & \\
\hline Minimum gap & $10.5 \mathrm{~mm}$ & & \\
\hline Minimum range of gap taper & $0-2 \mathrm{mrad}$ & & \\
\hline Effective field, $B_{\text {eff }}$ at $11.5 \mathrm{~mm}$ gap & $>0.750 \mathrm{~T}$ & 3) & $0.803 \pm 0.007 \mathrm{~T}$ \\
\hline Effective field, $B_{\text {eff }}$ at $10.5 \mathrm{~mm}$ gap & $>0.835 \mathrm{~T}$ & 4) & $0.891 \pm 0.009 \mathrm{~T}$ \\
\hline Peak field, $B_{\text {peak }}$ at $10.5 \mathrm{~mm}$ gap & $>0.849 \mathrm{~T}$ & 5) & $0.906 \pm 0.009 \mathrm{~T}$ \\
\hline Effective $K$ value, $K_{e f f}$ at $10.5 \mathrm{~mm}$ gap & $>2.57$ & 4) & $2.74 \pm 0.03$ \\
\hline Peak $K$ value, $K_{\text {peak }}$ at $10.5 \mathrm{~mm}$ gap & $>2.62$ & 5) & $2.79 \pm 0.03$ \\
\hline First harmonic energy & $<3.2 \mathrm{keV}$ & 6) & $2.96 \pm 0.05 \mathrm{keV}$ \\
\hline $\begin{array}{l}\text { Rms peak magnetic field error at } 11.5 \\
\text { mm gap }\end{array}$ & $<0.5 \%$ & $3,7)$ & $0.49 \pm 0.07 \%$ \\
\hline Rms phase error at $11.5 \mathrm{~mm}$ gap & $<8^{\circ}$ & $3,8)$ & $4.0 \pm 0.7^{\circ}$ \\
\hline
\end{tabular}

\footnotetext{
1) Average quantities and rms variations were calculated from 23 measured IDs at the listed gaps. The undulator period length $(3.3 \mathrm{~cm})$ derived from the magnetic measurements is accurate to better than 3 decimals. Average value and rms variation was not calculated.

2) To be exact, the device has 144 poles $=71.5$ periods. Magnets at the ends are not of full strength. In this document, for all spectral calculations except power calculations, $B_{\text {eff }}$ and $N-2=70$ periods were used. For power calculations, however, a conservative value of $B_{\text {peak }}$ and $N=72$ periods was used.

3) Vendor specification. All magnetic specifications to vendor were at $11.5 \mathrm{~mm}$ gap.

4) Calculated based on specifications to vendor.

5) Calculated. There was no specification on $B_{\text {peak }}$.

6) Derived from the effective $K$ value at $10.5 \mathrm{~mm}$ (zero emittance calculation).

7) On the average, the specified value for the rms peak magnetic field error was met. However, although a design parameter, this is not an important parameter. See Appendix B, Figure B6 for gap-dependent measurements.

8) The rms phase error was met by a large margin. In fact, the rms phase error does not exceed $7.1^{\circ}$ for any device at any gap. See Appendix B, Figure B5 for gap-dependent measurements and phase-error definition.
} 
The undulators were tuned in the magnetic measurement facility at the APS for high spectral purity at seven gaps ranging from $10.5 \mathrm{~mm}$ to $30.0 \mathrm{~mm}$. The measured gap dependency of the magnetic field is summarized in Table 2. Additional measured gapdependent magnetic properties can be found in Appendix B.

Table 2: Measured gap dependency of the peak magnetic field $B_{\text {peak }}$ and effective field $B_{\text {eff. }}$ The average values for 23 IDs are shown. The effective $K$ value $K_{\text {eff }}$ and the first harmonic energy $E_{l}$ are derived from $B_{\text {eff }}$, and the powers are derived from $B_{\text {peak }}$.

\begin{tabular}{l|llllll}
\hline $\begin{array}{l}\text { Gap } \\
(\mathrm{mm})\end{array}$ & $\mathrm{B}_{\text {peak }}(\mathrm{T})^{3)}$ & $\mathrm{B}_{\text {eff }}(\mathrm{T})^{4)}$ & $\mathrm{K}_{\text {eff }}$ & $\mathrm{E}_{1}(\mathrm{keV})^{5)}$ & $\begin{array}{l}\mathrm{P}_{\text {density }}{ }^{6)} \\
\left(\mathrm{kW} / \mathrm{mrad}^{2}\right)\end{array}$ & $\begin{array}{l}\mathrm{P}_{\text {total }}{ }^{6} \\
(\mathrm{~kW})\end{array}$ \\
\hline & & & & & & \\
10.5 & $0.9056 \pm 0.0090$ & $0.8906 \pm 0.0087$ & $2.744 \pm 0.027$ & $2.959 \pm 0.046$ & 168.0 & 6.04 \\
11.0 & 0.8582 & 0.8455 & 2.605 & 3.209 & 159.0 & 5.42 \\
11.5 & $0.8132 \pm 0.0070$ & $0.8027 \pm 0.0067$ & $2.473 \pm 0.021$ & $3.474 \pm 0.043$ & 150.6 & 4.87 \\
12.0 & 0.7715 & 0.7625 & 2.349 & 3.750 & 142.6 & 4.38 \\
12.5 & 0.7319 & 0.7243 & 2.232 & 4.040 & 135.1 & 3.95 \\
13.0 & 0.6944 & 0.6880 & 2.120 & 4.343 & 128.0 & 3.55 \\
13.5 & $0.6587 \pm 0.0055$ & $0.6535 \pm 0.0052$ & $2.014 \pm 0.016$ & $4.658 \pm 0.049$ & 121.2 & 3.20 \\
14.0 & 0.6257 & 0.6212 & 1.914 & 4.979 & 114.9 & 2.88 \\
14.5 & 0.5943 & 0.5905 & 1.820 & 5.310 & 109.0 & 2.60 \\
15.0 & 0.5645 & 0.5613 & 1.730 & 5.650 & 103.3 & 2.35 \\
15.5 & $0.5361 \pm 0.0045$ & $0.5336 \pm 0.0043$ & $1.644 \pm 0.013$ & $5.996 \pm 0.055$ & 97.9 & 2.12 \\
16.0 & 0.5100 & 0.5078 & 1.565 & 6.340 & 92.9 & 1.91 \\
17.0 & 0.4614 & 0.4599 & 1.417 & 7.036 & 83.7 & 1.57 \\
18.0 & 0.4174 & 0.4165 & 1.283 & 7.733 & 75.3 & 1.28 \\
18.5 & $0.3970 \pm 0.0033$ & $0.3963 \pm 0.0032$ & $1.221 \pm 0.010$ & $8.077 \pm 0.056$ & 71.4 & 1.16 \\
19.0 & 0.3781 & 0.3776 & 1.163 & 8.409 & 67.9 & 1.05 \\
20.0 & 0.3430 & 0.3427 & 1.056 & 9.054 & 61.2 & 0.87 \\
24.5 & $0.2211 \pm 0.0019$ & $0.2215 \pm 0.0019$ & $0.682 \pm 0.006$ & $11.437 \pm 0.037$ & 37.0 & 0.36 \\
25.0 & 0.2107 & 0.2111 & 0.651 & 11.638 & 34.8 & 0.33 \\
30.0 & $0.1303 \pm 0.0011$ & $0.1310 \pm 0.0012$ & $0.404 \pm 0.004$ & $13.039 \pm 0.018$ & 17.5 & 0.12 \\
35.0 & 0.0806 & 0.0812 & 0.250 & 13.672 & 7.7 & 0.05 \\
40.0 & 0.0498 & 0.0504 & 0.155 & 13.933 & 3.1 & 0.02 \\
& & & & & & \\
\hline & & & & & & \\
\hline
\end{tabular}

1) The measured gaps are shown in italic with rms variations added at those gaps only. Intermediate gap values were obtained by linear interpolation of log of field vs. gap.

2) Fields were not measured for gaps larger than $30.0 \mathrm{~mm}$. For gaps beyond $30.0 \mathrm{~mm}$, the dependency on the gap was extrapolated from the field at the two largest measured gaps $(24.5 \mathrm{~mm}$ and $30.0 \mathrm{~mm})$.

3) The measured peak magnetic field is defined as the average of the absolute value of the magnetic field at the poles in the regular part of the undulator, i.e., omitting 5 end-poles at each end of the undulator.

4) The measured effective magnetic field is derived from the slope of the slippage between the electron and the light in the regular part of the undulator at the optimum view angle that defines the undulator axis for on-axis radiation $(\theta=0)$. The slippage is proportional to $1+K_{\text {eff }}^{2} / 2$. (The $K_{\text {eff }}^{2} / 2$ is a measure of the electron's average angular deflection $\left\langle x^{\prime 2}\right\rangle$ from the undulator axis.) This definition of $K_{\text {eff }}$ ensures that spectral harmonics calculated from a single harmonic sinusoidal variation of the magnetic field, i.e., an ideal field, overlap in energy with the harmonics derived numerically from the measured magnetic field. 
The effective magnetic field $B_{e f f}$ is calculated from the relation $K_{e f f}=0.934 \lambda_{u}(\mathrm{~cm}) \mathrm{B}_{e f f}(T)$, where $\lambda_{u}$ is the undulator period length $(3.3 \mathrm{~cm})$. Note that $B_{\text {eff }}$ becomes slightly larger than $B_{\text {peak }}$ at large gaps, which can be attributed to small angular trajectory deviations (on the average) with respect to the on-axis direction. In general, even for other definitions of $B_{\text {eff }}$, such as from a Fourier decomposition of the magnetic field, $B_{\text {eff }}$ may be larger than $B_{\text {peak }}$, e.g., for a "flat-top" shape of the field. See Appendix B for definition of $B_{e f f}$.

5) Zero-emittance calculation using $K_{\text {eff }}$ for on-axis radiation $(\theta=0)$ for beam energy $7.0 \mathrm{GeV}$. The values here differ from what is measured on the beamline. There will be an asymmetric broadening of the spectrum due to the beam emittance and the slit defining the angular acceptance of the photon beam that causes a downward energy shift of the peak intensity. The numerically calculated energy shift for the present lattice and a small aperture $<$ size of the central cone is $\frac{\Delta E_{1}}{E_{1}} \sim 5 * 10^{-3}$.

6) Zero-emittance calculation at beam energy $7.0 \mathrm{GeV}$ and current $100 \mathrm{~mA}$, using $B_{\text {peak }}$ and the full number of undulator periods $(N=72)$.

The average over 23 measured IDs of the effective magnetic field $B_{\text {eff }}$ vs. gap is shown in Figure 1, and the corresponding effective $K$ value $K_{\text {eff }}$ and the zero emittance calculated value for the first harmonic energy $E_{l}$ are shown in Figure 2. The error bars indicate the rms spread.

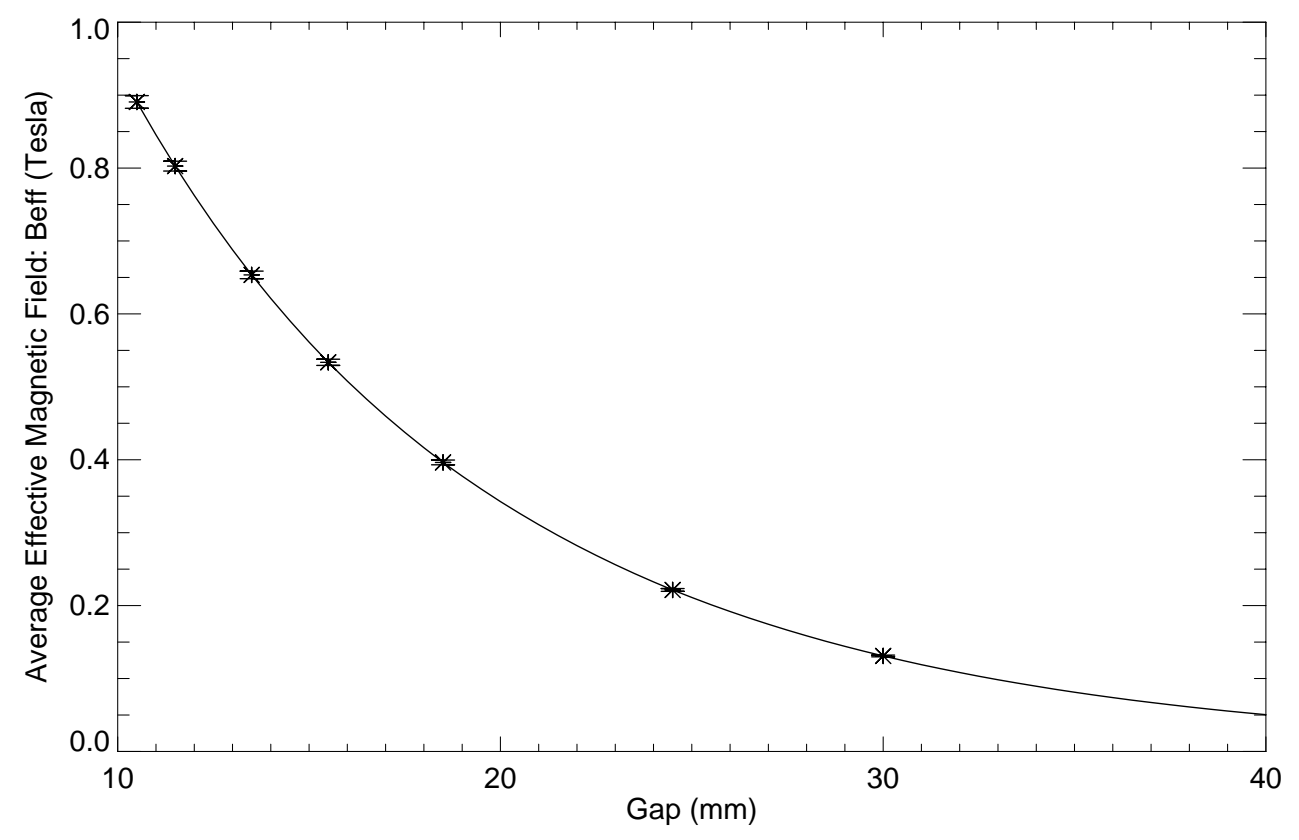

Figure 1. Measured effective magnetic field $B_{\text {eff }}$ as a function of gap (minimum gap is $10.5 \mathrm{~mm}$ ). The error bars show the rms spread over 23 measured IDs. The data are listed in Table 2. The solid line is the interpolation/extrapolation of field vs. gap. The measured value of $0.80 \mathrm{~T}$ at $11.5 \mathrm{~mm}$ gap exceeds the vendor specification $(0.75 \mathrm{~T})$ by a large margin. 


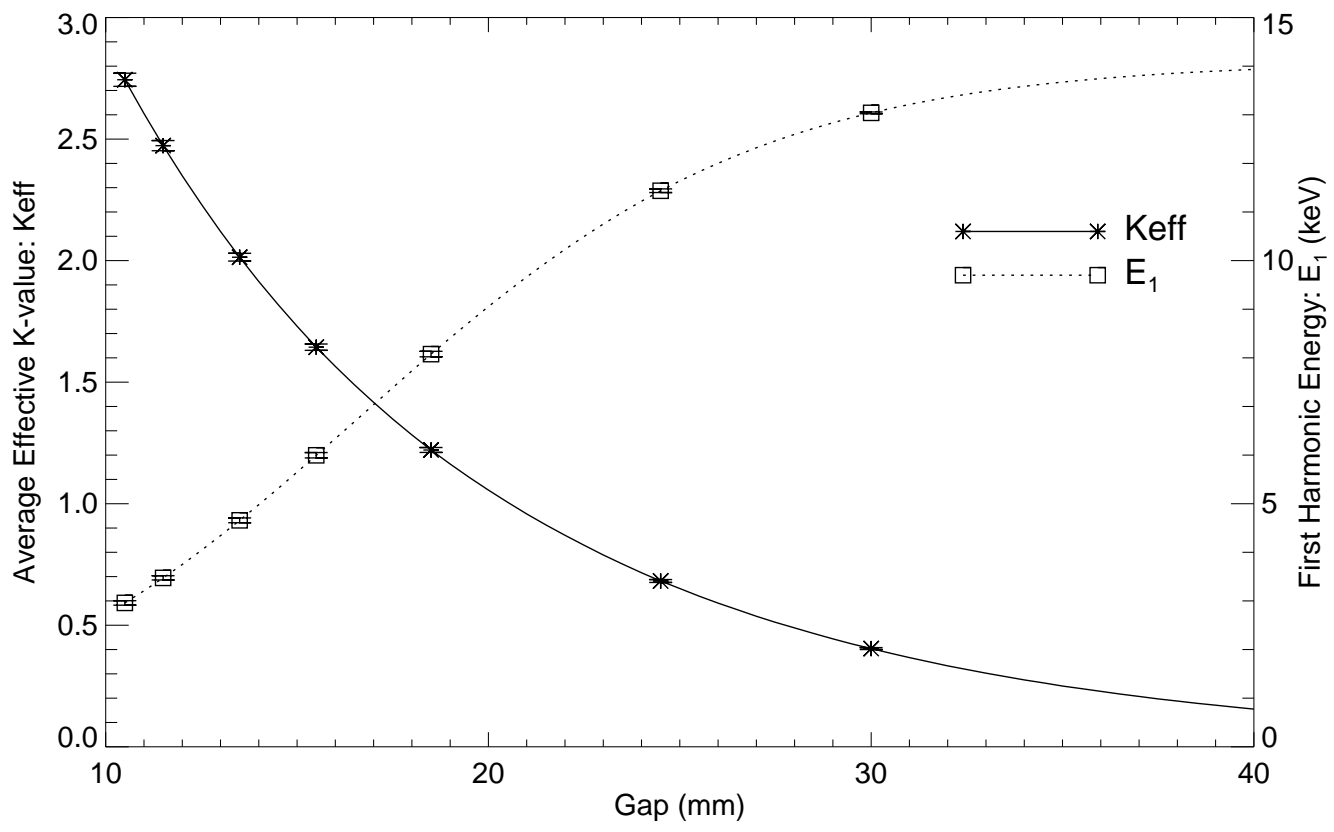

Figure 2. Measured effective $K$ value and calculated first harmonic energy $\left(E_{l}\right)$ for onaxis radiation for $7.0 \mathrm{GeV}$ beam energy as a function of gap. The error bars show the rms spread over 23 measured IDs. The data are listed in Table 2. The solid line is the interpolation/extrapolation of $K$ vs. gap, and the dotted line is calculated from the interpolated/extrapolated values. The minimum gap is $10.5 \mathrm{~mm}\left(K_{\text {eff }}=2.744\right)$, and the minimum calculated energy is $2.96 \mathrm{keV}$. The useful tuning range for the first harmonic is approximately $3-13 \mathrm{keV}$. 


\section{Spectral Performance} by

The radiated photon energy $E_{n}$ for the $n^{\text {th }}$ harmonic for a single electron is given

$$
E_{n}(k e V)=\frac{0.95 E^{2}(G e V) n}{\lambda_{u}(\mathrm{~cm})\left(1+K_{\text {eff }}^{2} / 2+\gamma^{2} \theta^{2}\right)},
$$

where $E$ is the beam energy, $\gamma$ is the relativistic factor for electrons, $\gamma=1957 E(\mathrm{GeV}), \lambda_{\mathrm{u}}$ is the undulator period length, $K_{\text {eff }}=0.934 \lambda_{u}(\mathrm{~cm}) B_{\text {eff }}(T)$ is the effective $K$ value, and $\theta$ is the polar angle measured from the undulator axis.

The tuning of the energy for permanent magnet devices relies on changing the undulator gap, hence changing the effective magnetic field or effective $K$ value. The minimum accessible energy depends on the undulator period length. For an Undulator A, $\sim 3 \mathrm{keV}$ can be reached in the first harmonic at a minimum gap of $10.5 \mathrm{~mm}$. The tuning curves are calculated numerically by tracing the peak intensity vs. the harmonic energy for a given set of beam and undulator parameters for an ideal magnetic field. (There is an asymmetric broadening of the spectral harmonics due to the beam emittance, and a small downward energy shift of the peak intensity from the single-electron formula is observed.)

The beam energy spread, which is especially important for narrow bandwidth harmonics at high energies because it broadens the harmonics, was taken into account. The reduction in brilliance due to magnetic field errors is not taken into account, but it can be estimated separately. Based on measurements of absolute flux and the comparison with calculations using the measured magnetic field that were reported earlier [7, 9], we expect to obtain at least $90 \%$ of the ideal intensity for the third harmonic and at least $80 \%$ for the fifth harmonic for small apertures, i.e., on-axis brilliance (aperture $<<$ size of central cone). The corresponding numbers for large apertures, on the order of the size of the central cone, are $95 \%$ for the third and $90 \%$ for the fifth harmonic.

\section{On-Axis Brilliance Tuning Curves}

The calculated on-axis brilliance tuning curves for Undulator A for the present low-emittance lattice are compared in Figure 3 to the APS design lattice. (The APS storage ring lattices and beam parameters used in the calculations are discussed in Appendix A.) The on-axis brilliance was increased by more than one order of magnitude due to changes that were made to the lattice, and the highest brilliance today is well over $10^{19} \mathrm{ph} / \mathrm{s} / \mathrm{mrad}^{2} / \mathrm{mm}^{2} / 0.1 \%$ bw. 


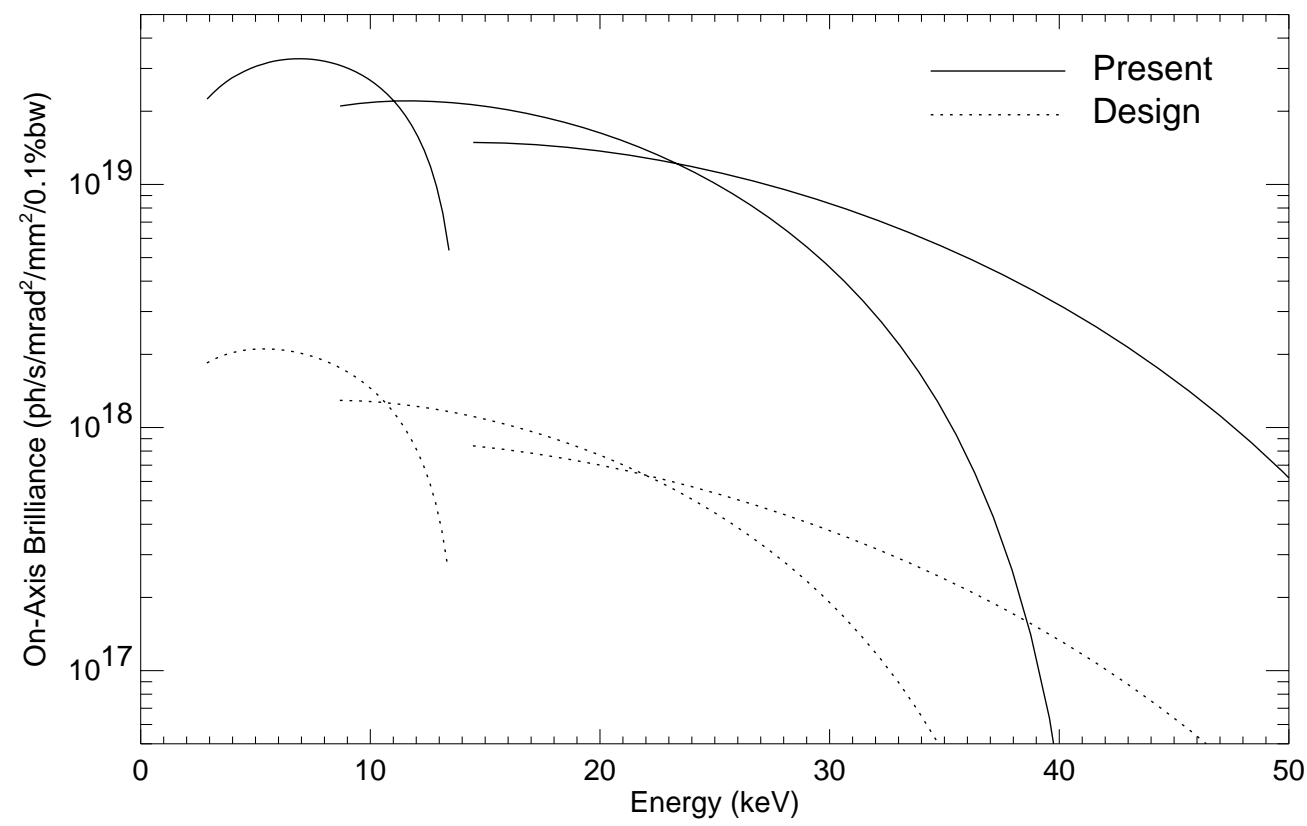

Figure 3. Calculated on-axis brilliance tuning curves for the first, third, and fifth harmonics for the present low-emittance lattice in comparison with the APS design lattice for a beam energy of $7.0 \mathrm{GeV}$ and a current of $100 \mathrm{~mA}$. The minimum gap is $10.5 \mathrm{~mm}$ and the lowest reachable energy is $3.0 \mathrm{keV}$. The on-axis brilliance was increased by more than one order of magnitude due to three changes to the APS lattice: the emittance was reduced from from $8.2 \mathrm{~nm}$-rad to $3.5 \mathrm{~nm}$-rad, the coupling was reduced from $10 \%$ to $1 \%$, and the vertical $\beta$ function was reduced from $10 \mathrm{~m}$ to $4 \mathrm{~m}$ in the ID straight sections. The highest brilliance is $3.3 \times 10^{19} \mathrm{ph} / \mathrm{s} / \mathrm{mrad}^{2} / \mathrm{mm}^{2} / 0.1 \% \mathrm{bw}$ at $7 \mathrm{keV}$ for the first harmonic.

\section{On-Axis Flux Tuning Curves}

The flux integrated over the full size of the central cone does not depend on the beam emittance. However, for a fixed-sized aperture that does not cover the whole central cone, the flux will increase with the smaller emittance. (The small increase is due to the narrowing of the central cone.)

The on-axis flux tuning curve for the present low-emittance lattice is compared in Figure 4 with the design lattice for a fixed aperture of $2.5 \mathrm{~mm}(\mathrm{~h}) \times 1.0 \mathrm{~mm}$ (v) at $30 \mathrm{~m}$ from the source. This aperture was near optimal for the design lattice but is somewhat bigger than the FWHM-size for the low-emittance lattice. (See also Figures 7 - 10, which give examples of spatial photon distributions at $30 \mathrm{~m}$ for the two lattices.) For the chosen aperture, there is a $30 \%$ increase in the flux at the peak in the tuning curve (at $5 \mathrm{keV}$ for the first harmonic). The flux at the APS is approaching $10^{15} \mathrm{ph} / \mathrm{s} / 0.1 \% \mathrm{bw}$ (a value that depends on the chosen aperture). 


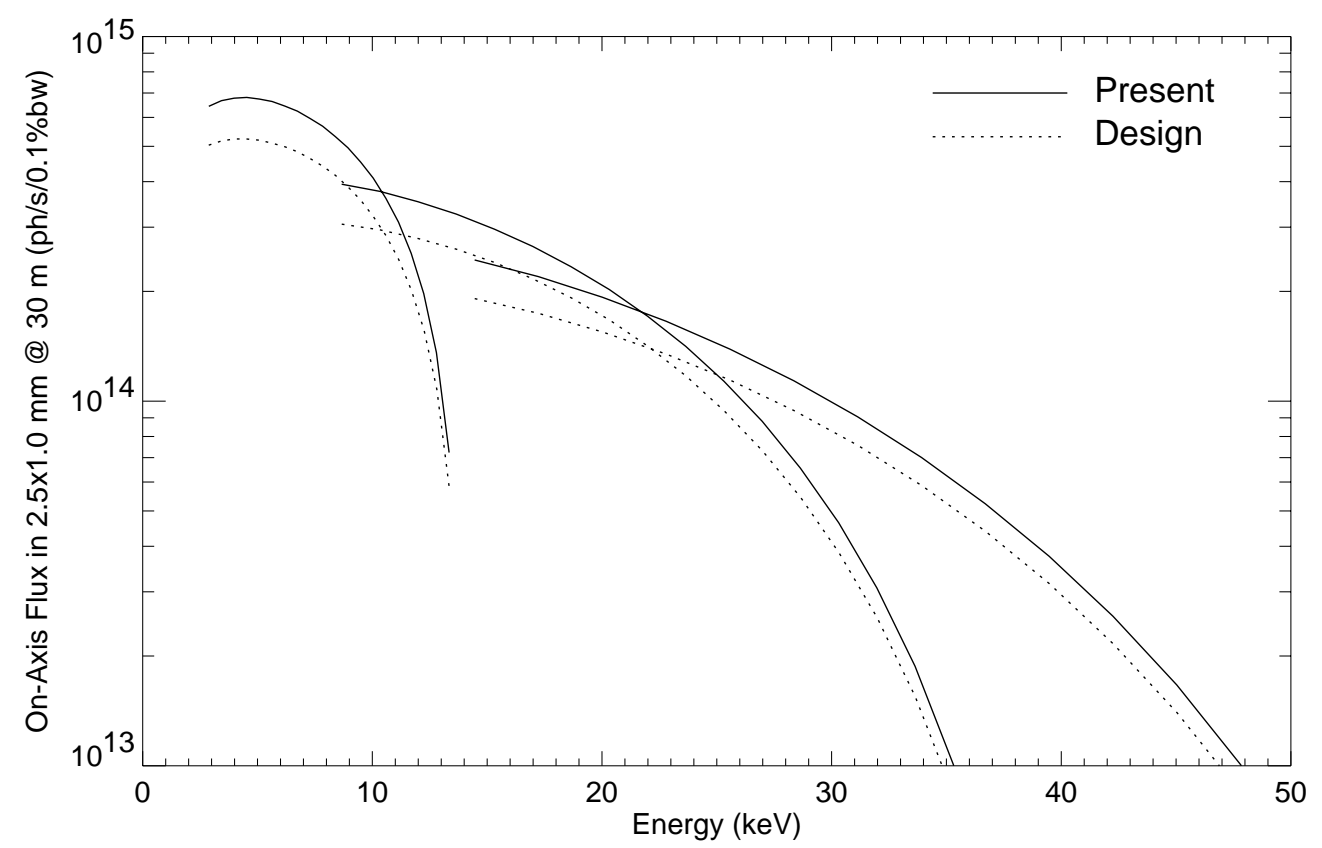

Figure 4. Calculated on-axis flux tuning curves for the first, third, and fifth harmonics for the present low-emittance lattice in comparison with the APS design lattice for a beam energy of $7.0 \mathrm{GeV}$ and a current of $100 \mathrm{~mA}$. The minimum gap is $10.5 \mathrm{~mm}$ and the lowest reachable energy is $3.0 \mathrm{keV}$. The fixed-size aperture is $2.5 \mathrm{~mm}(\mathrm{~h}) \times 1.0 \mathrm{~mm}(\mathrm{v})$ at $30 \mathrm{~m}$ from the source. The highest flux is $6.8 \times 10^{14} \mathrm{ph} / \mathrm{s} / 0.1 \% \mathrm{bw}$ at $5 \mathrm{keV}$ for the first harmonic. (It is $30 \%$ higher than for the design lattice due to the narrowing of the central cone.)

\section{Canted and Tandem IDs Compared with Undulator A}

Shorter and longer IDs are being evaluated for the straight sections. (Two shorter canted IDs, with an electron trajectory bend in between them, can make two sources in a single straight section.) Here we compare three different lengths of IDs with the $3.3-\mathrm{cm}$ period length: $L=2.1 \mathrm{~m}$ represents shorter canted IDs, $L=2.4 \mathrm{~m}$ is for the current length of Undulator A, and $L=4.8 \mathrm{~m}$ represents placing two Undulators A in tandem. We kept the length at $4.8 \mathrm{~m}$ to represent two full-length Undulators $\mathrm{A}$ in series with perfect phasing, although some space will be needed between them for hardware (magnetic phase shifter) to maintain the synchronism condition between the electron and light when the undulator gap is changed.

\section{On-Axis Brilliance Tuning Curves for Canted and Tandem IDs}

The on-axis brilliance tuning curves for the three devices are shown in Figure 5 and the brilliances are compared in Table 3 for energies selected from each harmonic and at $7 \mathrm{keV}$, where the peak in the tuning curve occurs. The on-axis brilliance scales with 
the number of periods $N^{p}$ as expected, and the exponent $p$ approaches one for high energies and long devices when $\sigma_{r}$ ' becomes $\ll<\sigma_{y^{\prime}}$. (See Appendix A, where there is a discussion of the brilliance scaling from estimates of the flux in the central cone.)

Table 3: Calculated on-axis brilliance ${ }^{1)}$ for three different ID lengths with period length $3.3 \mathrm{~cm}$ for the present low-emittance lattice. The exponent $p$ for scaling vs. the number of periods $N^{p}$ is given in parenthesis with Undulator A $(N=70)$ being the reference. ${ }^{2)}$

\begin{tabular}{|c|c|c|c|}
\hline Energy (keV) & $\begin{array}{l}L=2.4 \mathrm{~m} ; N=70 \\
\text { (Undulator A) }\end{array}$ & $\begin{array}{l}L=2.1 \mathrm{~m} ; N=60 \\
\text { (Canted) }\end{array}$ & $\begin{array}{l}L=4.8 \mathrm{~m} ; N=142 \\
\text { (Tandem) }\end{array}$ \\
\hline $7 \quad\left(1^{\text {st }}\right)$ & $3.29 \times 10^{19}$ & $2.61 \times 10^{19}(1.50)$ & $8.69 \times 10^{19}(1.37)$ \\
\hline $10 \quad\left(1^{\mathrm{st}}\right)$ & $2.60 \times 10^{19}$ & $2.07 \times 10^{19}(1.48)$ & $6.76 \times 10^{19}(1.35)$ \\
\hline $20 \quad\left(3^{\text {rd }}\right)$ & $1.62 \times 10^{19}$ & $1.33 \times 10^{19}(1.28)$ & $3.61 \times 10^{19}(1.13)$ \\
\hline $30 \quad\left(5^{\text {th }}\right)$ & $8.71 \times 10^{18}$ & $7.27 \times 10^{18}$ & $1.86 \times 10^{19}(1.07)$ \\
\hline
\end{tabular}

1) On-axis brilliance in units of $\mathrm{ph} / \mathrm{s} / \mathrm{mrad}^{2} / \mathrm{mm}^{2} / 0.1 \%$ bw at $7.0 \mathrm{GeV}$ beam energy and $100 \mathrm{~mA}$ current.

${ }^{2)}$ Use $N^{p}$ to estimate the on-axis brilliance for other lengths.

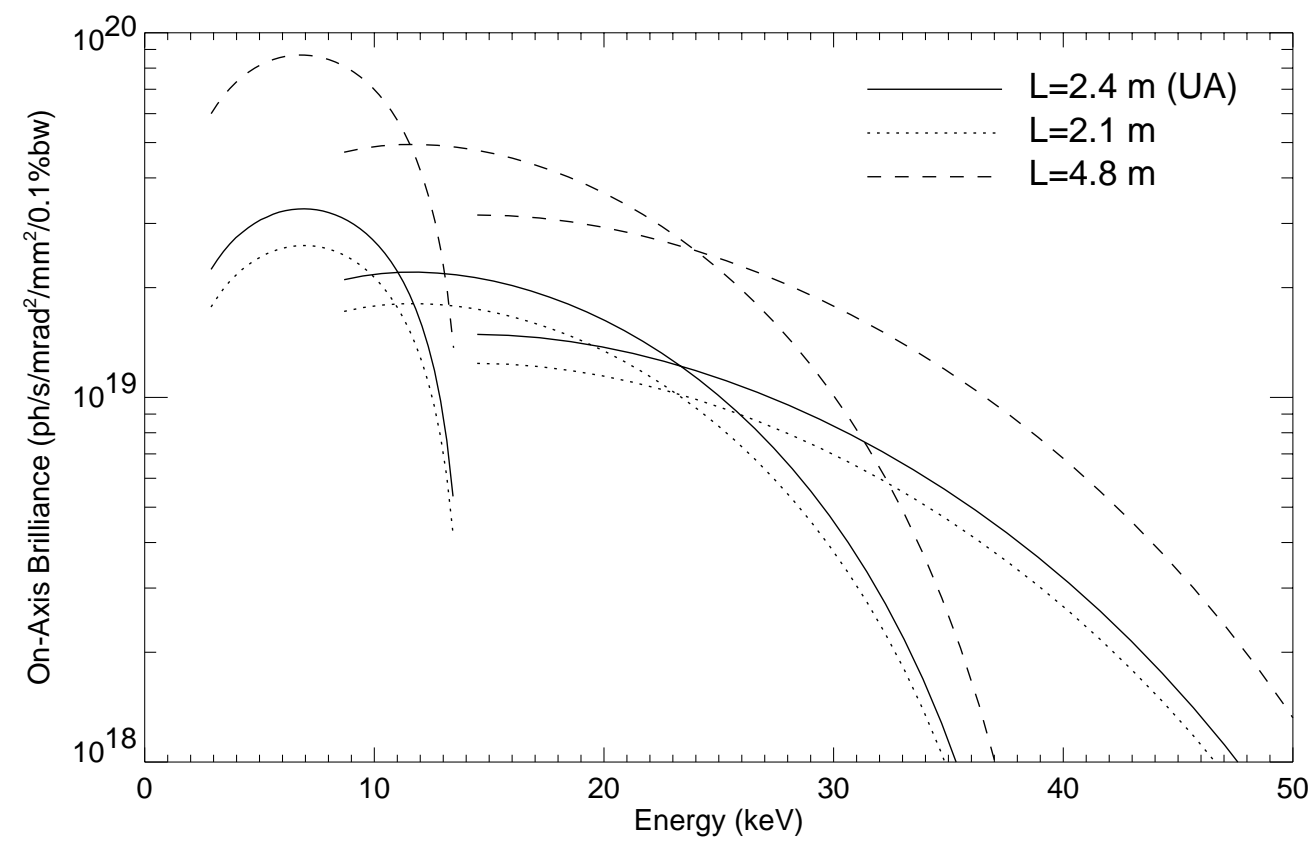

Figure 5. Calculated on-axis brilliance tuning curves for the first, third, and fifth harmonics for the present low-emittance lattice comparing three lengths of IDs with period length $3.3 \mathrm{~cm}: L=2.1 \mathrm{~m}, L=2.4 \mathrm{~m}$ (Undulator A; same as the solid curve in Figure 3), and $L=4.8 \mathrm{~m}$. The beam energy is $7.0 \mathrm{GeV}$ and the current is $100 \mathrm{~mA}$. The minimum gap is $10.5 \mathrm{~mm}$ and the lowest reachable energy is $3.0 \mathrm{keV}$. 


\section{On-Axis Flux Tuning Curves for Canted and Tandem IDs}

The on-axis flux tuning curves for the same devices for an aperture of $2.5 \mathrm{~mm}(\mathrm{~h})$ x $1.0 \mathrm{~mm}(\mathrm{v})$ at $30 \mathrm{~m}$ from the source are shown in Figure 6 and compared in Table 4 for select energies. The peak in the flux tuning curve appears at slightly lower energy ( 5 $\mathrm{keV})$. The flux scales almost linearly with the number of periods $N$, as expected.

Table 4: Calculated on-axis flux ${ }^{1)}$ for three different ID lengths with period length $3.3 \mathrm{~cm}$ for the present low-emittance lattice. The exponent $p$ for scaling vs. the number of periods $N^{p}$ is given in parenthesis with Undulator A $(N=70)$ being the reference. ${ }^{2)}$

\begin{tabular}{lllll}
\hline Energy $(\mathrm{keV})$ & $\begin{array}{l}L=2.4 \mathrm{~m} ; N=70 \\
(\text { Undulator A) }\end{array}$ & $\begin{array}{l}L=2.1 \mathrm{~m} ; N=60 \\
(\text { Canted })\end{array}$ & $\begin{array}{l}L=4.8 \mathrm{~m} ; N=142 \\
\text { (Tandem) }\end{array}$ \\
\hline & & & & \\
5 & $\left(1^{\mathrm{st}}\right)$ & $6.81 \times 10^{14}$ & $5.63 \times 10^{14}(1.23)$ & $1.51 \times 10^{15}(1.13)$ \\
10 & $\left(1^{\mathrm{st}}\right)$ & $4.09 \times 10^{14}$ & $3.46 \times 10^{14}(1.09)$ & $8.60 \times 10^{14}(1.05)$ \\
20 & $\left(3^{\text {rd }}\right)$ & $2.02 \times 10^{14}$ & $1.71 \times 10^{14}(1.08)$ & $4.30 \times 10^{14}(1.07)$ \\
30 & $\left(5^{\text {th }}\right)$ & $9.06 \times 10^{13}$ & $7.67 \times 10^{13}(1.08)$ & $1.92 \times 10^{14}(1.06)$
\end{tabular}

1) On-axis flux in units of $\mathrm{ph} / \mathrm{s} / 0.1 \% \mathrm{bw}$ at $7.0 \mathrm{GeV}$ beam energy and $100 \mathrm{~mA}$ current.

2) Use $N^{p}$ to estimate the on-axis flux for other lengths; note $\mathrm{p} \sim 1$.

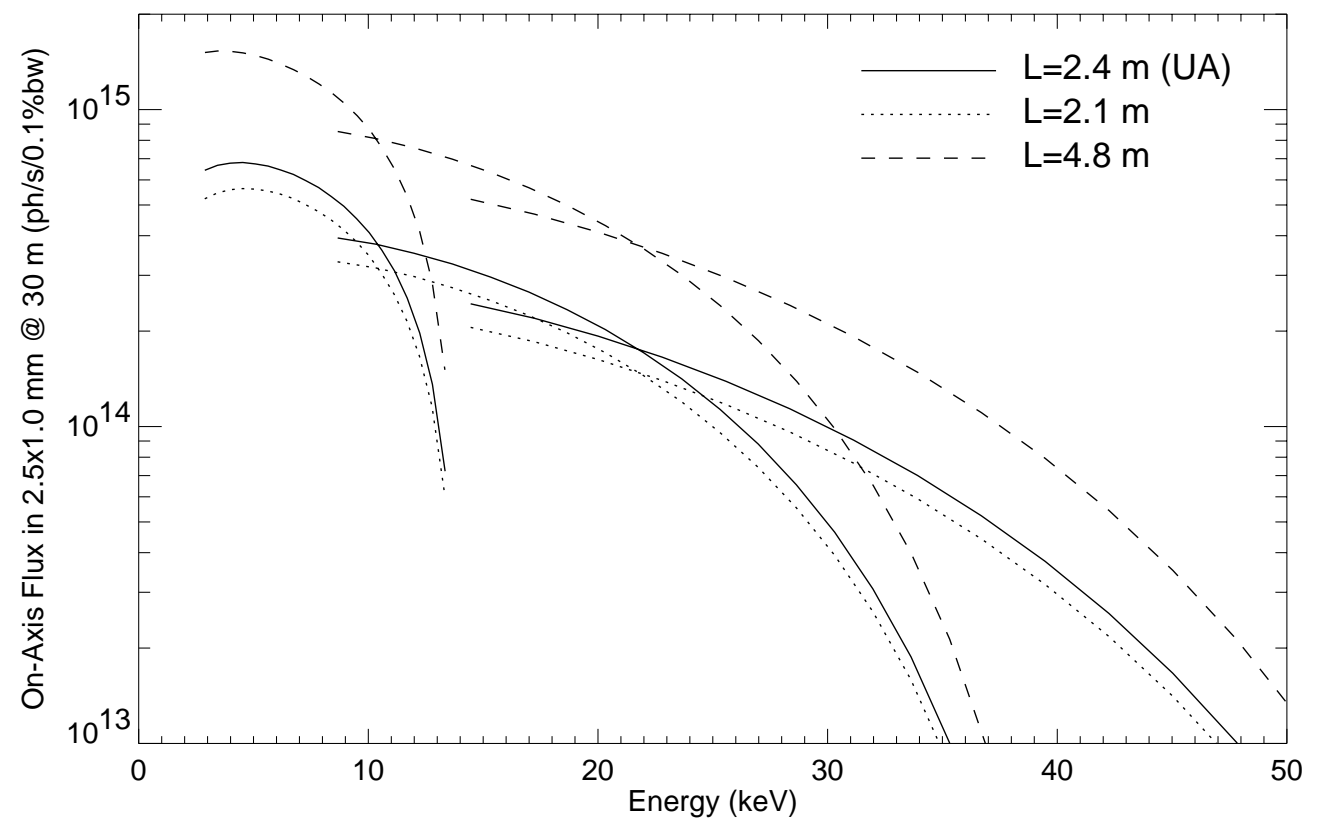

Figure 6. Calculated on-axis flux tuning curves for the first, third, and fifth harmonics for the present low-emittance lattice comparing three lengths of IDs with period length

$3.3 \mathrm{~cm}: L=2.1 \mathrm{~m}, L=2.4 \mathrm{~m}$ (Undulator A; same as solid curve in Figure 4), and $L=4.8$ $\mathrm{m}$. The aperture is $2.5 \mathrm{~mm}(\mathrm{~h}) \times 1.0 \mathrm{~mm}(\mathrm{v})$ at $30 \mathrm{~m}$ from the source. The beam energy is $7.0 \mathrm{GeV}$ and the current is $100 \mathrm{~mA}$. The minimum energy is $3.0 \mathrm{keV}$ at $10.5 \mathrm{~mm}$ gap. 


\section{Power Considerations}

The immense power $(\sim 6 \mathrm{~kW})$ and power densities $\left(\sim 160 \mathrm{~kW} / \mathrm{mrad}^{2}\right)$ generated by the Undulator A insertion devices at small gaps were an engineering challenge in the initial design stage of the APS front-end components. And it continues to be so as the demand for higher brilliance and flux continues, and longer IDs and higher stored beam current are being considered. (The radiated total power and the on-axis power density both scale with the number of periods $N$ and the ring current $I$.) The total power is also proportional to the square of both the beam energy and the peak magnetic field (for an ideal field), whereas the on-axis power density is proportional to the fourth power of the beam energy and depends linearly on the peak magnetic field.

The power calculations in this document have been performed using the peak magnetic field $B_{\text {peak }}$ and the full number of periods $N$ in the analytical formula rather than $N-2$ and $B_{e f f}$ that were used for the brilliance calculations. The undulator power angular distribution and its dependency on different parameters are described in the paper by Kim [12]. By making this choice, for the Undulator A parameters, we will typically overestimate both the total emitted power, i.e., integrated over all energies and all angles, and the on-axis power density by $\sim 5 \%$ at $10.5 \mathrm{~mm}$ gap (The error was derived from numerical calculations using the measured magnetic field; in fact, using $N-2$ and $B_{\text {eff }}$ would give an almost perfect agreement, but we have chosen to present calculations using conservative values.) At larger gaps, $B_{\text {peak }} \sim B_{\text {eff }}$ and the discrepancy will be less.

The series of spatial photon distributions that were given in the earlier technical bulletin are still applicable for getting an idea of the complex distribution outside of the central cone (Figures 6 - 8 in ANL/APS/TB-17 [2]) and will not be repeated here. The central cone of radiation will be narrower for a smaller emittance (low-emittance lattice), but the effect of the emittance on the undulator power angular distribution can be neglected. Both of these issues will be discussed here. The aperture-limited power curves in TB-17 (Figures 11 - 19) are still applicable and are very important for beamline designers. The flux curves in the same figures will change for the smaller emittance and should be re-examined for detailed designs. (The gap-dependent information in Figure $10 \mathrm{a}$ changed, but Figures $10 \mathrm{~b}$ - c can be used.) 


\section{Angular Distribution and Effect of Emittance}

Photons are emitted in discrete annular regions outside of the central cone. These rings belong to higher harmonics, but their energy is the same as the central-cone energy because they are observed at off-axis angles. The polar angle is given by

$$
\theta=\frac{1}{\gamma} \sqrt{\frac{l}{n}\left(1+K_{e f f}^{2} / 2\right)}
$$

where $n$ is the harmonic number for the central cone energy, and $l$ is the next higher harmonic number (counting from $n$ ). The photon distribution for the first harmonic energy $(n=1, E=2.95 \mathrm{keV})$ at $30 \mathrm{~m}$ from the source for $K_{\text {eff }}=2.74$ (closed gap 10.5 $\mathrm{mm}$ ) is illustrated in Figure 7 for the $8.2 \mathrm{~nm}-\mathrm{rad}$ design lattice. The second harmonic $(l=1)$ is visible near $\pm 4 \mathrm{~mm}$ in the vertical direction. The corresponding cross-section profiles of the central cone are shown in Figure 8.

The corresponding photon distributions and cross-section profiles of the central cone for the low-emittance lattice are shown in Figure 9 and Figure 10, respectively. The peak intensity grows and the central cone becomes narrower, and the second harmonic now appears more distinctly.

The rms size of the central cone at distance $D$ from the source can be approximated by

$$
D \sqrt{\left(\sigma_{x^{\prime}, y^{\prime}}^{2}+\left(\frac{\sigma_{x, y}}{D}\right)^{2}+\sigma_{r^{\prime}}^{2}\right)},
$$

where $\sigma_{x^{\prime}, y^{\prime}}$ is the rms beam divergence and $\sigma_{x, y}$ is the rms beam size in the horizontal and vertical directions, respectively. The diffraction-limited photon-beam divergence is $\sigma_{r^{\prime}}$.

The off-axis annuli of radiation are normally not of interest for the users, however, at detuned harmonic energies, they can be used as a sensitive tool to measure the beam parameters because of the small natural width of the annular regions [9]. 

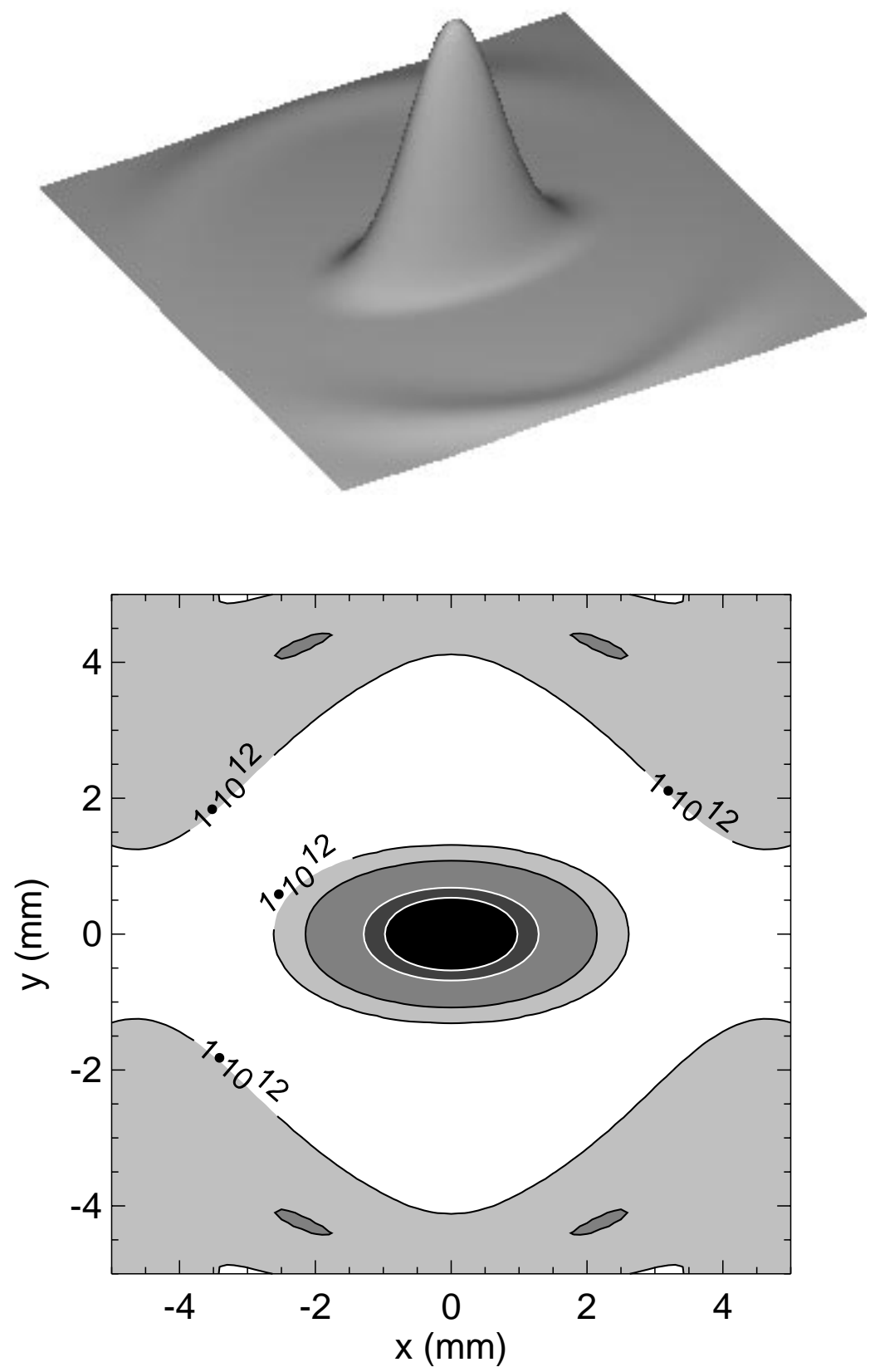

Figure 7. Spatial photon distribution at $30 \mathrm{~m}$ from the source for $K_{\text {eff }}=2.74$ (closed gap $10.5 \mathrm{~mm})$ at the first harmonic energy $(2.95 \mathrm{keV})$ for the $8.2 \mathrm{~nm}$-rad design lattice. The peak intensity is $3.4 \times 10^{14} \mathrm{ph} / \mathrm{s} / \mathrm{mm}^{2} / 0.1 \% \mathrm{bw}$. The innermost contour line is the FWHM of the central cone. The second innermost white contour line is at the $10^{14}$ level (other contour lines are a factor of 10 apart). 

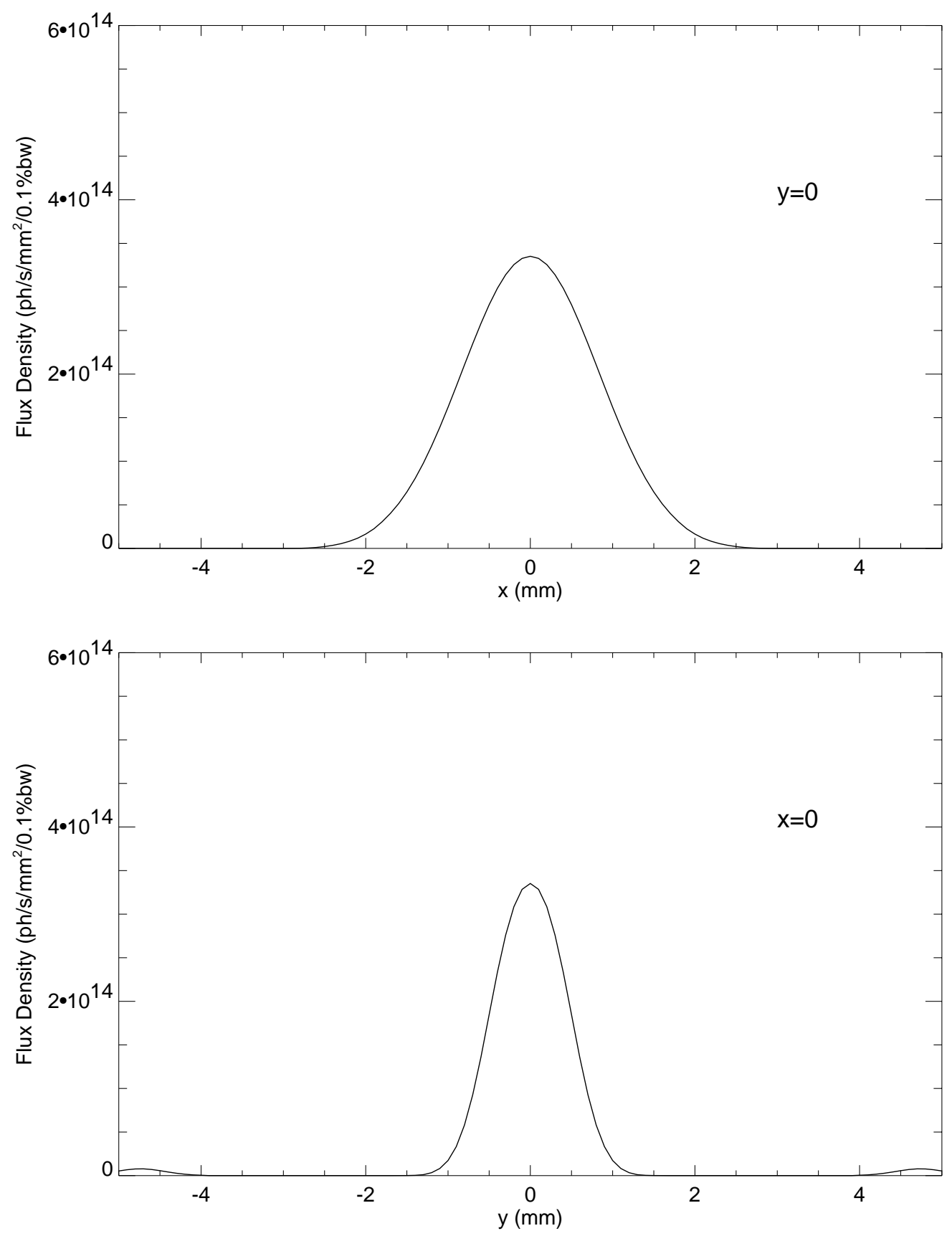

Figure 8. Flux density cross-section profiles in the horizontal and vertical directions at $30 \mathrm{~m}$ from the source corresponding to the distribution shown in Figure 7 at the first harmonic energy $(2.95 \mathrm{keV})$ for the $8.2 \mathrm{~nm}$-rad design lattice. The $\mathrm{FWHM}_{\mathrm{x}}$ is $1.95 \mathrm{~mm}$ and the $\mathrm{FWHM}_{\mathrm{y}}$ is $1.07 \mathrm{~mm}$. 

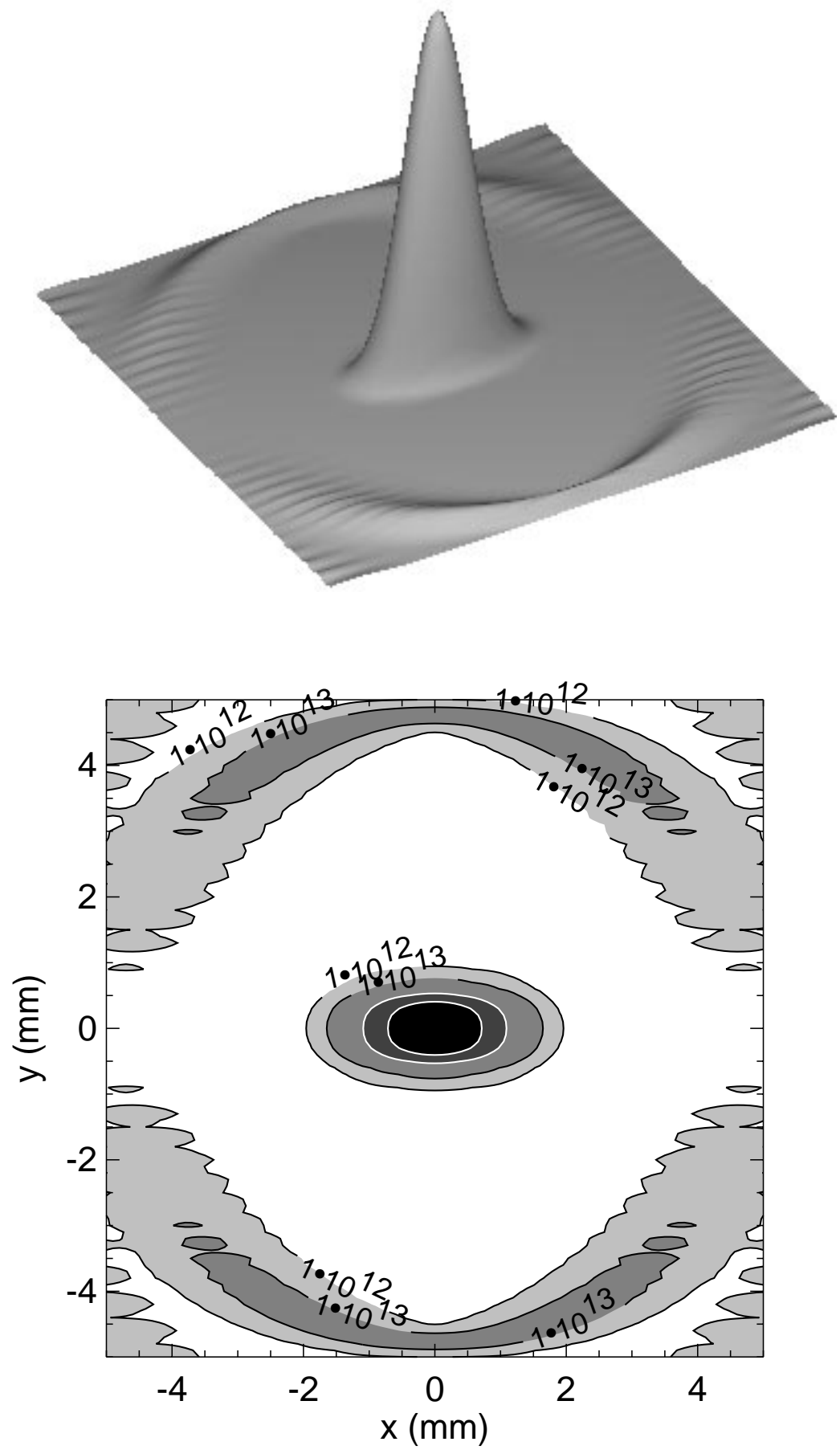

Figure 9. Spatial photon distribution at $30 \mathrm{~m}$ from the source for $K_{\text {eff }}=2.74$ (closed gap $10.5 \mathrm{~mm})$ at the first harmonic energy $(2.95 \mathrm{keV})$ for the present $3.5 \mathrm{~nm}$-rad lowemittance lattice. The peak intensity is $5.1 \times 10^{14} \mathrm{ph} / \mathrm{s} / \mathrm{mm}^{2} / 0.1 \% \mathrm{bw}$. The innermost contour line is the FWHM of the central cone. The second innermost white contour line is at the $10^{14}$ level (other contour lines are a factor of 10 apart). The central cone, including the second harmonic off-axis, appears more distinctly for the smaller emittance. (The jaggedness is partially an artifact from the calculations due to the finite number of points used.) 

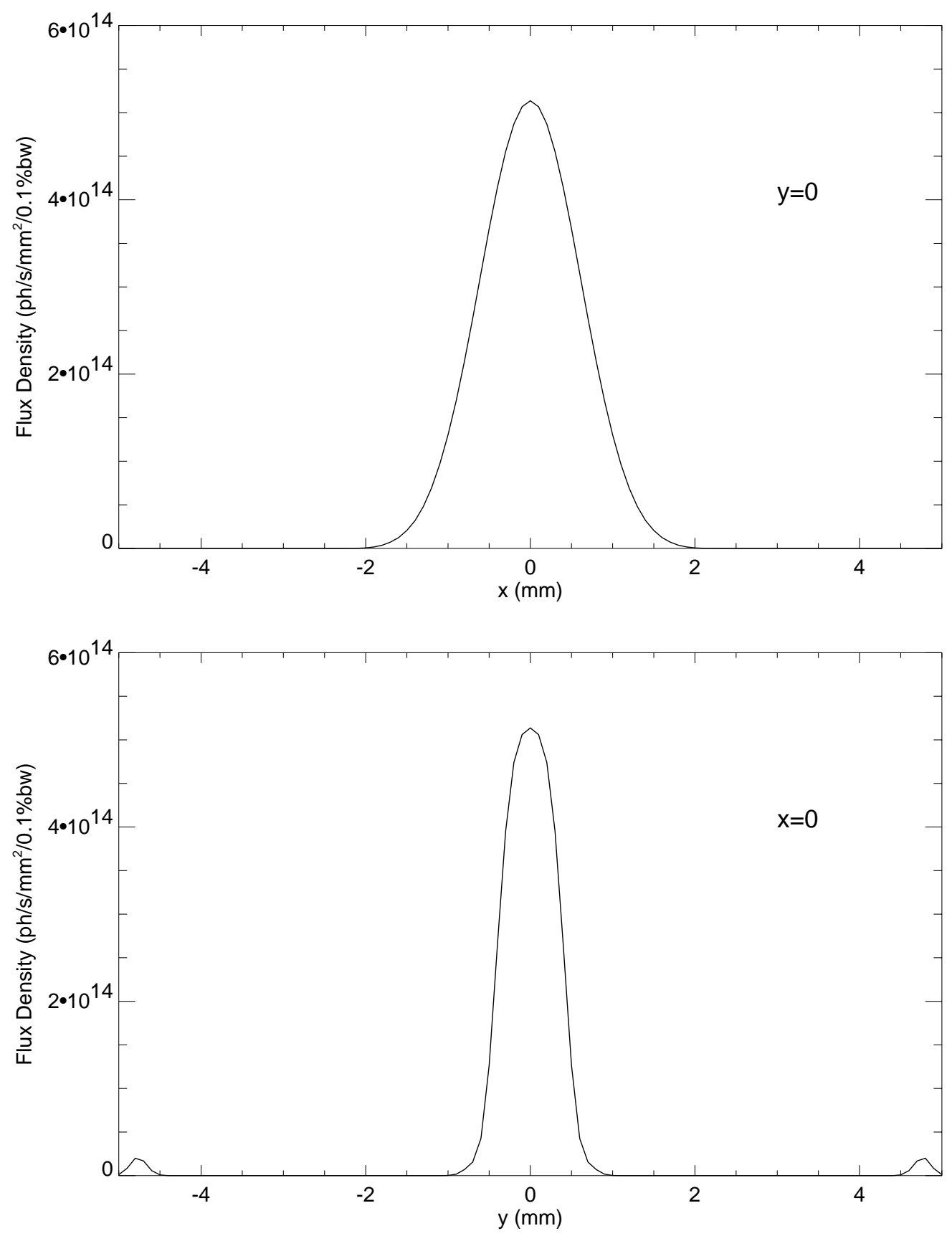

Figure 10. Flux density cross-section profiles in the horizontal and vertical directions at $30 \mathrm{~m}$ from the source corresponding to the distribution shown in Figure 9 at the first harmonic energy $(2.95 \mathrm{keV})$ for the $3.5 \mathrm{~nm}$-rad low-emittance lattice. The $\mathrm{FWHM}_{\mathrm{x}}$ is $1.81 \mathrm{~mm}$ and the $\mathrm{FWHM}_{\mathrm{y}}$ is $0.94 \mathrm{~mm}$. 
The frequency-integrated angular power distribution is very wide in comparison with the size of the central cone for any given selected harmonic energy $[12,13]$ and is typically not sensitive to the beam emittance. This is illustrated in Figure 11 where three examples of power distributions at select energies and the frequency-integrated power for Undulator A at closed gap $(10.5 \mathrm{~mm})$ at $30 \mathrm{~m}$ from the source is shown in a series of frames calculated for the low-emittance lattice. The size of the aperture was increased to $\pm 10 \mathrm{~mm}$ to fully cover the much wider power density profile.

The distribution at the first harmonic energy $(2.95 \mathrm{keV})$ is shown in the upper left frame (same as in Figure 9 but with the wider aperture here). The second and third harmonics are clearly visible outside the central cone. A similar distribution at the third harmonic energy $(8.85 \mathrm{keV})$ is shown in the lower left frame. The fourth, fifth, and sixth harmonics are intense enough to appear now. The intensity pattern outside the central cone becomes more complex, partially due to the emittance that mixes the harmonics.

The upper right frame shows the interesting (and well-known) distribution at a highly detuned energy near the third harmonic (detuning $0.5 \mathrm{keV}$; same vertical scale). The central cone of radiation splits into two peaks that are separated in the vertical direction-here they moved off-center by $\pm 1.1 \mathrm{~mm}$, which is well beyond the central cone for the third harmonic energy. The distributions on the high-energy side of a harmonic energy are different, however (not shown). The intensity in the central cone disappears quickly, whereas the intensity of the outer rings of radiation changes slowly with energy. The outer rings consisting of closely spaced higher harmonics (spacing $\sim 1 / \sqrt{n}$ ) are very important as they are responsible for contributing to the power density outside of the central cone when integrated over all energies (frequencies).

The frequency-integrated power is built up of profiles similar to those presented in Figure 11 to about five times the critical energy $(\sim 150 \mathrm{keV}$ for the Undulator A at closed gap). The composite is shown in the lower right frame in the same figure. The useful flux in the central cone contains only a small fraction of the total power $(<10 \%$ for the example chosen). The angular width of the frequency-integrated power density profile is approximately $\pm K_{\text {peak }} / \gamma$ in the horizontal direction and $\pm 1 / \gamma$ in the vertical direction $(1 / \gamma=73 \mu \mathrm{rad}$ for $7.0 \mathrm{GeV})$. The effect of the APS beam emittance on the frequencyintegrated power density profile can therefore be ignored because $\sigma_{x^{\prime}}<<K_{\text {peak }} / \gamma$ and $\sigma_{y},<<1 / \gamma$. (This is in sharp contrast to the effect it has on the angular size of the central cone, and hence, the on-axis brilliance that was discussed above.) 


\section{$2.95 \mathrm{keV}$}
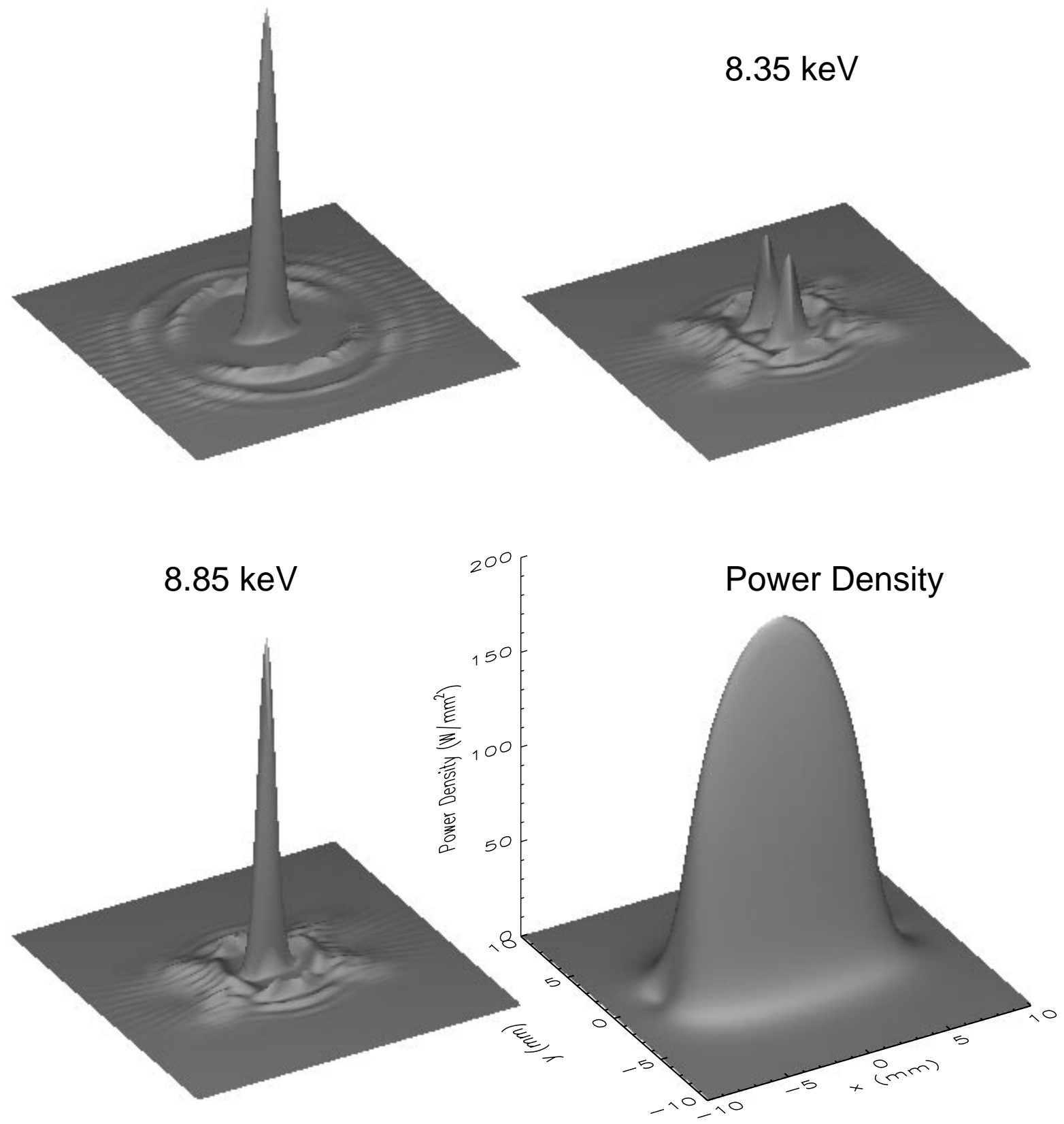

Figure 11. Examples of spectral power profiles and how they build up to the power density profile at $D=30 \mathrm{~m}$ from the source for Undulator A at closed gap $(10.5 \mathrm{~mm}$; $K_{\text {eff }}=2.74, K_{\text {peak }}=2.79$ ) for the low-emittance lattice. The aperture is $10 \times 10 \mathrm{~mm}$ and the vertical scale is the same for all select energies. The first harmonic energy is at $2.95 \mathrm{keV}$ (same distribution as in Figure 9), and the third harmonic is at $8.85 \mathrm{keV}$. The third harmonic was detuned by $0.5 \mathrm{keV}$, and this distribution is shown at $8.35 \mathrm{keV}$. Notice that the central cone split and that two relative strong peaks appear in the vertical direction. Note also the annular regions of intensity outside of the central cone that come from higher harmonics. The frequency-integrated power density is broad: $\sim \pm K_{\text {peak }} / \gamma$ and $\pm D / \gamma$ in the horizontal and vertical directions, respectively. 


\section{Spectral Power}

Beamline designers must know the spectral power content of the emitted radiation. To illustrate the importance of choosing a proper aperture, we calculated the angle-integrated spectral power (no limiting aperture) and the aperture-limited spectral power for Undulator A at closed gap $10.5 \mathrm{~mm}$ using $K_{\text {peak }}=2.79$ for beam energy 7.0 $\mathrm{GeV}$ and current $100 \mathrm{~mA}$. The results are shown in Figures 12 and 13, respectively.

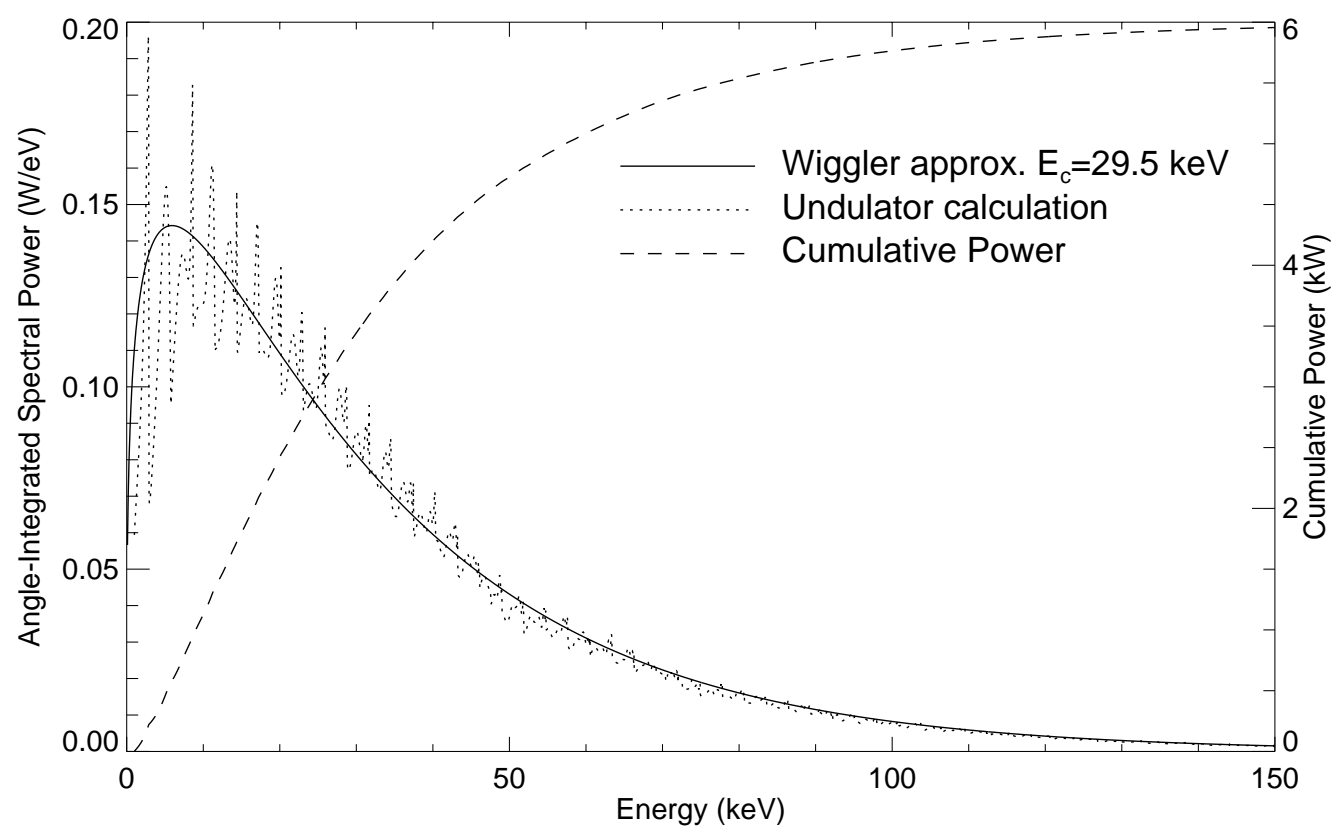

Figure 12. Angle-integrated spectral power (dotted curve) and cumulative power (dashed curve) for Undulator A at closed gap $10.5 \mathrm{~mm}$ for the present low-emittance lattice. (The cumulative power is the integral of the spectral power for the undulator calculation.) The wiggler approximation is also shown (solid curve) using a $K$ value of 2.79 , corresponding to the peak magnetic field and critical energy of $29.5 \mathrm{keV}$. Here, $E_{10 \%}=6 \mathrm{keV}, E_{50 \%}=25$ $\mathrm{keV}$, and $E_{90 \%}=72 \mathrm{keV}$. The $E_{50 \%}$ divides the integrated power curve in half, i.e., $50 \%$ of the power is below $25 \mathrm{keV}$ and $50 \%$ is between $25-150 \mathrm{keV}$. Note that $E_{50 \%}$ is somewhat smaller than $E_{c}$. Because we are integrating over all angles and all energies, the value of $6 \mathrm{~kW}$ corresponds to the total emitted power. The shape of the spectral power curve reflects the large content of higher harmonics being taken into account at large angles and the average becomes "wiggler-like."

The cumulative power (frequency-integrated power) calculated from the undulator curve is also shown, and we give the energies that divide the spectral power into different fractions of emitted power. (The cumulative power calculated from the wiggler approximation is not shown, but it does not differ from the undulator curve.) The calculated value $E_{50 \%}$ differs from $E_{c}$ ( $E_{c}$ divides the spectral power into half for a bending magnet but not for the undulator spectrum or wiggler-approximated spectrum). 
The critical energy $E_{c}$ is related to the beam energy and peak magnetic field by

$$
E_{c}(k e V)=0.665 E^{2}(G e V) B_{\text {peak }}(T),
$$

where $E$ is the beam energy $(7.0 \mathrm{GeV})$.

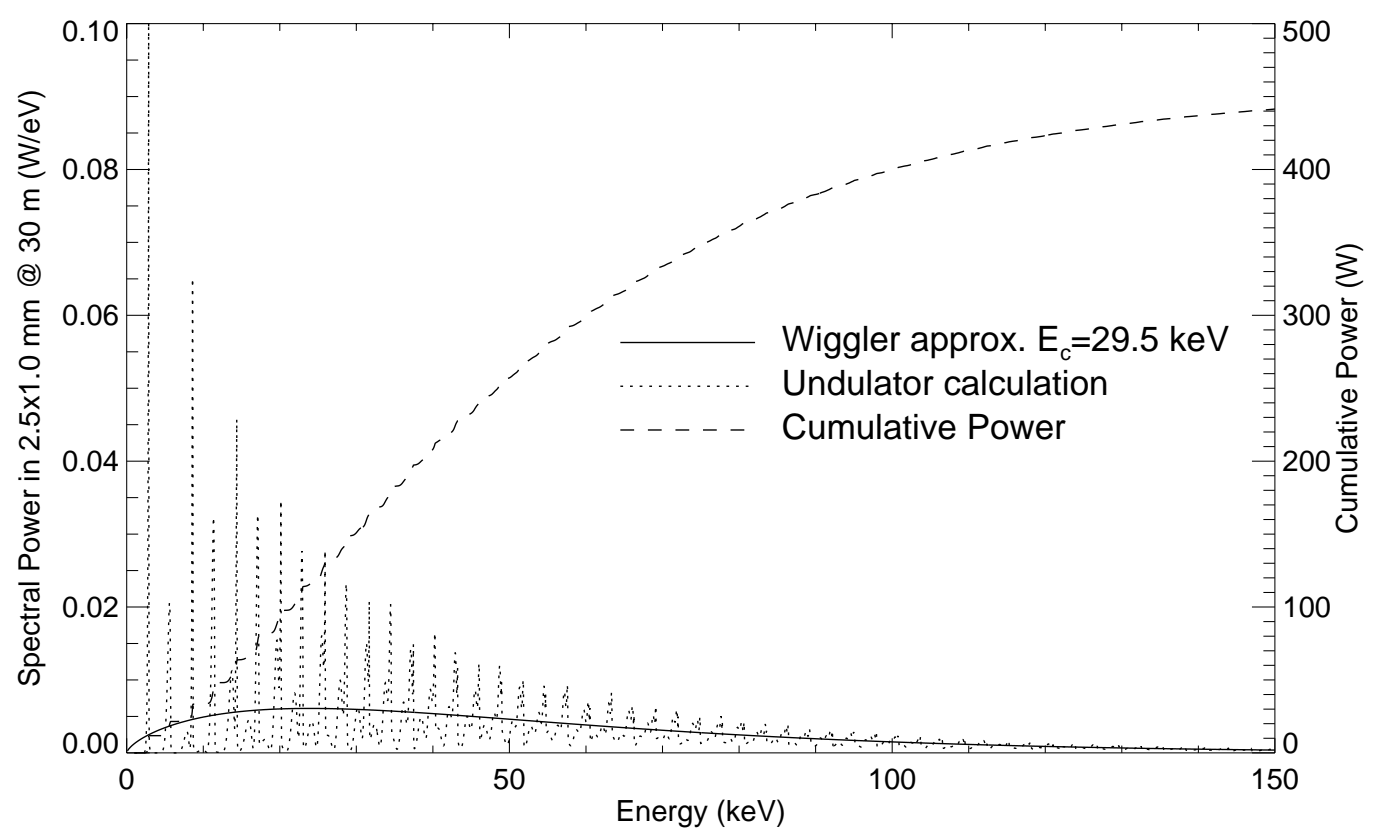

Figure 13: Aperture-limited spectral power (dotted curve) and cumulative power (dashed curve) for Undulator A at closed gap $(10.5 \mathrm{~mm})$ for the present low-emittance lattice. The aperture is $2.5 \mathrm{~mm}(\mathrm{~h}) \times 1.0 \mathrm{~mm}(\mathrm{v})$ at $30 \mathrm{~m}$ from the source. The wiggler approximation is also shown (solid curve) using a $K$ value of 2.79 , corresponding to the peak magnetic field and a critical energy of $29.5 \mathrm{keV}$. Here, $E_{10 \%}=11 \mathrm{keV}, E_{50 \%}=43$ $\mathrm{keV}$, and $E_{90 \%}=105 \mathrm{keV}$. Note that $E_{50 \%}$ is larger than $E_{c}$ here, showing that the spectrum is "harder" than what is expected from a bending magnet spectrum. The frequency-integrated power of $440 \mathrm{~W}$ is less than $10 \%$ of the total emitted power.

\section{Total Power and Power Density}

The gap dependency of the total power and on-axis power density calculated from the peak magnetic field is shown in Figure 14. We also show the total power and on-axis power density vs. the first harmonic energy (Figure 15), which are useful when comparing powers for different devices (different period lengths). The data are from Table 2, and the effect of the emittance on the on-axis power density was ignored as discussed above. 


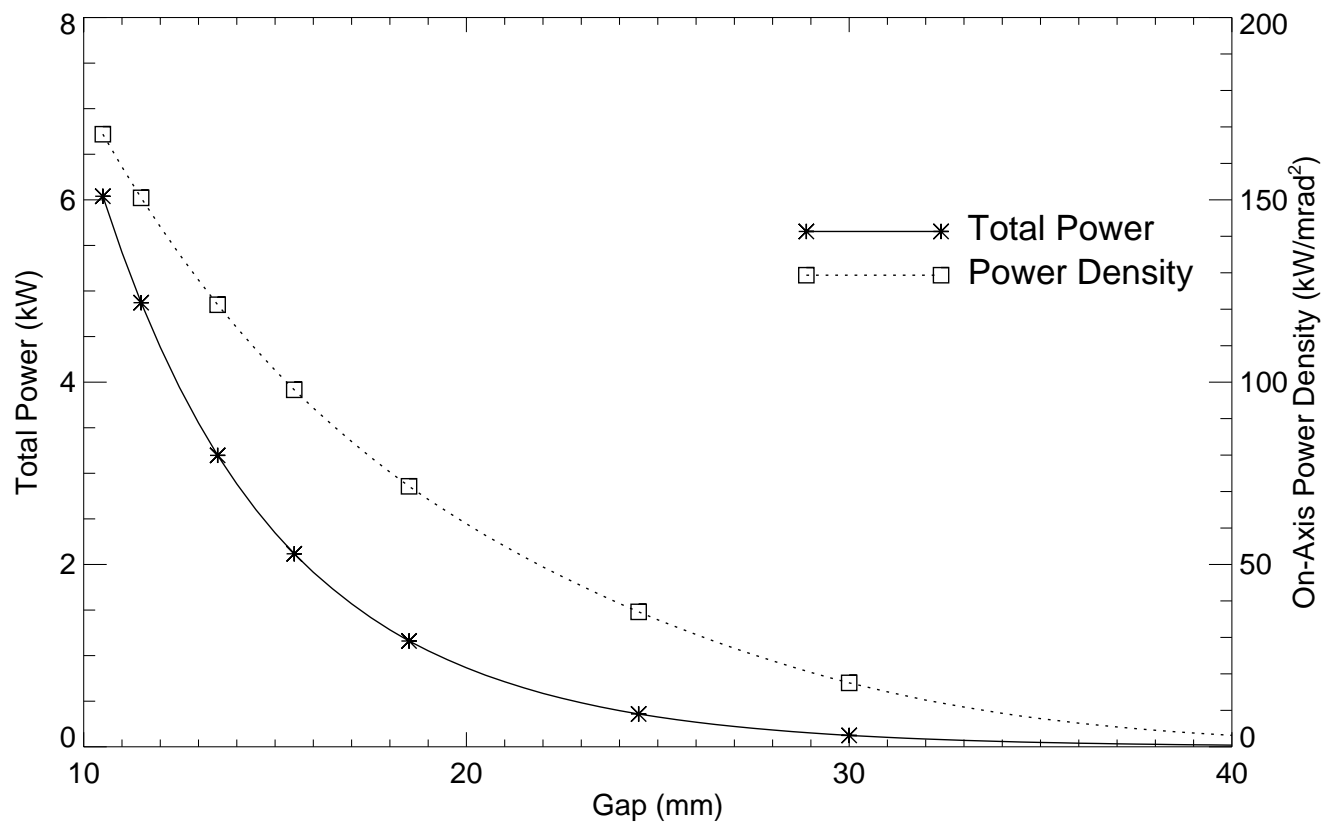

Figure 14. Total power and on-axis power density for Undulator A (zero-emittance calculation) vs. gap. The data are from Table 2. Beam energy is $7.0 \mathrm{GeV}$ and current is $100 \mathrm{~mA}$.

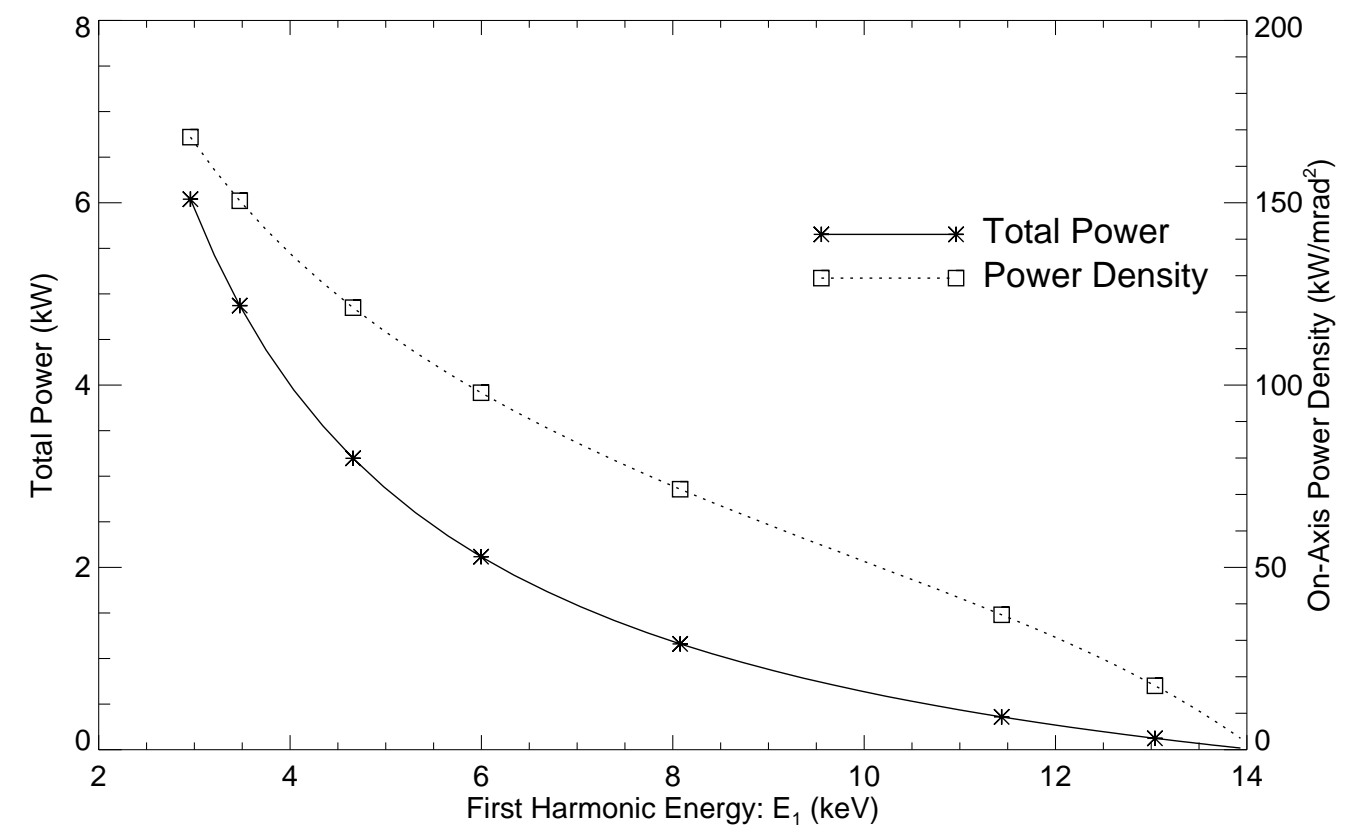

Figure 15. Total power and on-axis power density for Undulator A (zero-emittance calculation) vs. first harmonic energy. The data are from Table 2. Beam energy is 7.0 $\mathrm{GeV}$ and current is $100 \mathrm{~mA}$. 


\section{Prospect for Increased Brilliance}

There are several ways to increase the on-axis brilliance at the APS in the future, such as increasing the stored beam current, making the IDs longer, and making changes to the storage ring lattice [14]. With the storage ring hardware, it is technically feasible to operate at $300 \mathrm{~mA}$ current at $7.0 \mathrm{GeV}$ beam energy. However there are many technical and engineering tasks to be solved before this operation can be achieved. The main limiting factor is the increase in power loads on the front-end and first-optics beamline components that comes with increased current and longer devices. (Today, operating Undulators $A$ at a closed gap of $10.5 \mathrm{~mm}$ and at a beam energy of $7.0 \mathrm{GeV}$ limits the storage ring beam current to $130 \mathrm{~mA}$.)

\section{Beam and Lattice}

Here, we present the on-axis brilliance for the goal of operating the APS at 7.0 $\mathrm{GeV}$ and $300 \mathrm{~mA}$ using two suggested lattices with small coupling (0.1\%). The beam parameters for the lattices are listed in Appendix A, Table A3. One of the proposed lattices allows for longer straight sections $(10.7 \mathrm{~m})$, and, for this lattice, we used an undulator with $2.5 \mathrm{~cm}$ period length, which represents a near-optimum performance: tuning curves for first and third harmonics nearly intersect, and there is only a small brilliance drop between them (Figure 16).

\section{Insertion Device Changes}

One way to obtain higher brilliance is to use shorter period devices (shorter than $3.3 \mathrm{~cm}$ ). (As an example, the magnetic properties of a 2.7-cm-period device are given in Appendix C, and, in Appendix D, we compare the Undulator A on-axis brilliance vs. other devices at the APS for the present low-emittance lattice.) Both the total power and the on-axis power density will increase and may grow at a faster rate than the increase in the on-axis brilliance. It depends on the selected energy range and harmonic number, and studies will have to be done that make calculations for specific devices. For relatively open gaps (small $K$ values) and harmonic numbers above one, shorter period devices are favored. For operations at high currents (e.g., the proposed $300 \mathrm{~mA}$ ) and when using longer IDs, it will be necessary initially to restrict the tuning range to limit the power loads on the front-end and beamline components.

The on-axis brilliance tuning curves at a beam energy of $7.0 \mathrm{GeV}$ for the present low-emittance lattice are compared with the two future lattices in Figure 16 for different undulator lengths and different period lengths. The tuning curves were calculated up to $100 \mathrm{keV}$ and include very high harmonics (up to harmonic number 33 for Undulator A) to correctly trace the behavior at high energies. (Overlaps of the harmonics have been removed for clarity.) Note the degradation due to magnetic field errors was not taken into account, and the brilliances are therefore overestimated at high energies. (If the 
higher harmonics were completely smeared out, "wiggler-like" behavior, then the drop in brilliance at $100 \mathrm{keV}$ would be about a factor of two from what is shown in the figure.)

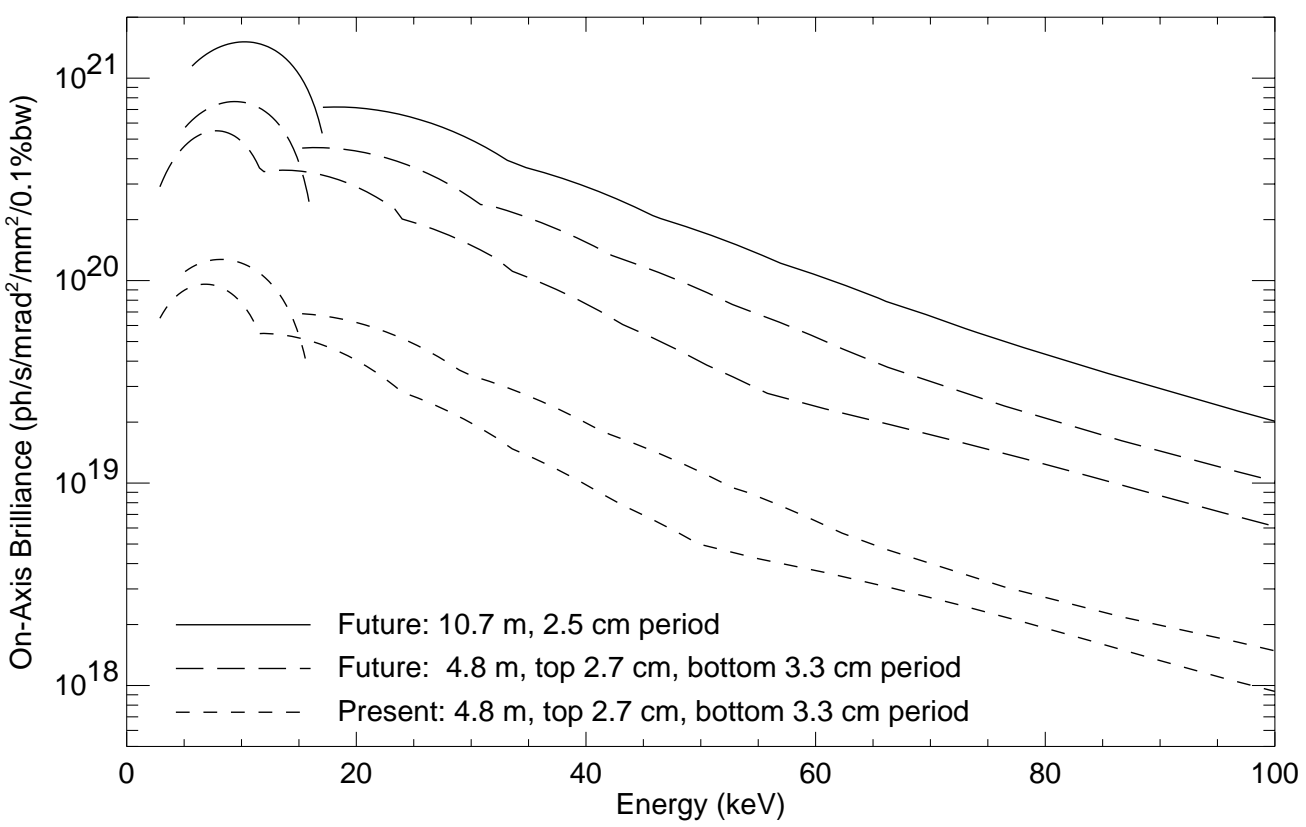

Figure 16. Calculated future on-axis brilliance tuning curves up to $100 \mathrm{keV}$ that include very high harmonics for three lattices and three undulator period lengths for a beam energy of 7.0 GeV. The minimum gaps are: $10.5 \mathrm{~mm}$ for $3.3-\mathrm{cm}$ period, $8.5 \mathrm{~mm}$ for 2.7 $\mathrm{cm}$ period, and $7.5 \mathrm{~mm}$ for $2.5-\mathrm{cm}$ period. The performance for $4.8-\mathrm{m}-$ long IDs for the present low-emittance lattice at $100 \mathrm{~mA}$ current are shown as short-dashed curves (at the bottom). The brilliance for the same IDs for the future lattice with $0.1 \%$ coupling at 300 $\mathrm{mA}$ are shown as long-dashed curves (in the middle). The top solid curve is for the future lattice with $0.1 \%$ coupling with a $10.7-\mathrm{m}-\mathrm{long}$ device and $2.5 \mathrm{~cm}$ period at 300 $\mathrm{mA}$. This ID would produce photons in excess of $10^{21} \mathrm{ph} / \mathrm{s} / \mathrm{mrad}^{2} / \mathrm{mm}^{2} / 0.1 \%$ bw at 10 $\mathrm{keV}$. At this photon energy, the total power would be about $30 \mathrm{~kW}$ ( 5 times Undulator A at closed gap) and the on-axis power density $1800 \mathrm{~kW} / \mathrm{mrad}^{2}(\sim 10$ times the Undulator A at closed gap). (At closed gap $7.5 \mathrm{~mm}$ for this device the corresponding values would be $80 \mathrm{~kW}$ and $3000 \mathrm{~kW} / \mathrm{mrad}^{2}$.)

\section{Acknowledgments}

The authors wish to thank L. Emery and M. Borland for providing the APS lattice model calculations and V. Sajaev for additional information on the APS $\beta$ functions. We are also thankful to $\mathrm{N}$. Vinokurov for valuable discussions on topics in beam and radiation physics. 


\section{Appendix A: The APS Storage Ring}

\section{Electron-Beam Phase Space}

The electron-beam transverse motion in the horizontal $\left(x, x^{\prime}\right)$ and vertical $\left(y, y^{\prime}\right)$ betatron phase space occupies paths on ellipses centered on the closed orbit that are periodic with the sector periodicity. To a good approximation, the phase space distribution is Gaussian

$$
f\left(x, x^{\prime}\right)=\frac{1}{2 \pi \varepsilon_{x}} \exp \left(-\frac{\gamma_{x}(s) x^{2}+2 \alpha_{x}(s) x x^{\prime}+\beta_{x}(s) x^{\prime 2}}{2 \varepsilon_{x}}\right),
$$

and similarly for $\left(y, y^{\prime}\right)$, where $\alpha(s), \beta(s)$, and $\gamma(s)$ are the so-called Twiss parameters that depend on the longitudinal coordinate $s$. The $\varepsilon_{\mathrm{x}}$ is referred to as the beam emittance [15]. Only two of the three Twiss parameters are independent because

$$
\gamma_{x}(s)=\frac{1+\alpha_{x}(s)^{2}}{\beta_{x}(s)}
$$

relates $\alpha, \beta$, and $\gamma$. The $\alpha$ function is related to $\beta$ through $\alpha_{x}(s)=-\frac{1}{2} \frac{d \beta_{x}(s)}{d s}$, and the variation of $\beta$ in free space between the focusing quadrupoles in the straight sections is given by

$$
\beta_{x}(s)=\beta_{x}\left(s_{o}\right)+\frac{\left(s-s_{o}\right)^{2}}{\beta_{x}\left(s_{o}\right)}
$$

where $\beta_{x}\left(s_{o}\right)$ is the minimum value of the $\beta$ function at location $s_{o}$.

The coupling constant $\chi$ describes the coupling between betatron oscillations in the two planes and is defined as the ratio of the vertical emittance to the horizontal emittance,

$$
\chi=\frac{\varepsilon_{y}}{\varepsilon_{x}}
$$

This value for today's operations is about $1 \%$, a much smaller value than that of the original design lattice specification of $10 \%$. For the APS, the natural emittance $\varepsilon$ is $\sim \varepsilon_{x}+$ $\varepsilon_{y}$. 
The horizontal rms beam size $\sigma_{x}$ and beam divergence $\sigma_{x^{\prime}}$ are usually expressed in the Twiss parameters that characterize the lattice,

$$
\sigma_{x}=\sqrt{\varepsilon_{x} \beta_{x}}, \quad \sigma_{x^{\prime}}=\sqrt{\varepsilon_{x} \gamma_{x}}
$$

and similarly for $\left(y, y^{\prime}\right)$. Note that, for a nontilted (upright) ellipse, $\gamma=1 / \beta$ and $\alpha=0$. The $\beta_{y}$ was decreased from $10 \mathrm{~m}$ in the original design lattice to $4 \mathrm{~m}$ in the center of the straight sections in order to increase the acceptance of the 5-m-long vacuum chambers and to increase the brilliance.

The electrons in the beam do not all have the same energy, and the beam energy spread $\delta E / E$ will create a distribution of orbits, each separated from the equilibrium closed orbit by an amount that is proportional to the energy difference. The spread of

orbits is characterized by the dispersion functions $\eta(s)$ and $\eta^{\prime}(s)\left[\eta^{\prime}(s)=\frac{d \eta(s)}{d s}\right]$ that have the same periodicity as the Twiss parameters. The energy dispersion widths are given by

$$
\delta_{x}(s)=\eta_{x}(s)\left(\frac{\delta E}{E}\right), \delta_{x^{\prime}}(s)=\eta_{x^{\prime}}(s)\left(\frac{\delta E}{E}\right)
$$

The total width of the beam is then the sum of the betatron widths and the energy dispersion widths,

$$
\sigma_{x}=\sqrt{\varepsilon_{x} \beta_{x}+\delta_{x}^{2}}, \quad \sigma_{x^{\prime}}=\sqrt{\varepsilon_{x} \gamma_{x}+\delta_{x^{\prime}}^{2}}
$$

and similarly for $\left(y, y^{\prime}\right)$. The dispersion $\eta_{x^{\prime}}$ is zero in the center of the straight sections for the new lattices but the $\eta_{x}$ is nonzero. The dispersion is always designed to be zero in the vertical direction, but perturbations in the optics may produce some small values.

\section{Photon-Beam Phase Space}

The phase space occupied by the undulator radiation is made up of two components, the electron-beam phase space and the diffraction-limited phase space of the photon beam-both can be approximated by Gaussian functions. The rms diffractionlimited photon-beam size $\sigma_{r}$ and divergence $\sigma_{r^{\prime}}$ at the undulator center are given by

$$
\sigma_{r}=\frac{\sqrt{2 \lambda L}}{4 \pi}, \sigma_{r^{\prime}}=\sqrt{\frac{\lambda}{2 L}}
$$


where $L$ is the undulator length and $\lambda$ is the radiated wavelength. The rms width of the radiated photon-beam phase-space distribution is then obtained from a convolution of the individual Gaussian distributions,

$$
\Sigma_{x}=\sqrt{\sigma_{x}^{2}+\sigma_{r}^{2}}, \quad \Sigma_{x^{\prime}}=\sqrt{\sigma_{x^{\prime}}^{2}+\sigma_{r^{\prime}}^{2}}
$$

and similarly for $\left(y, y^{\prime}\right)$.

For today's lattices, with a coupling of about $1 \%$, the photon-beam size in both $x$ and $y$ is dominated by the electron-beam size, and the $\sigma_{r}(\sim 2 \mu \mathrm{m}$ for $8 \mathrm{keV}$ photons; $L=2.4 \mathrm{~m}$ ) can be ignored. The photon-beam divergence, however, is dominated by the electron-beam divergence only in the horizontal direction. In the vertical direction, the natural divergence of the photons $\sigma_{r^{\prime}}(\sim 6 \mu \mathrm{rad}$ for $8 \mathrm{keV}$ photons; $L=2.4 \mathrm{~m})$ is comparable to the electron-beam divergence $(\sim 3 \mu \mathrm{rad})$ and needs to be taken into account.

\section{Brilliance Estimate from the Flux in the Central Cone}

It is useful to be able to estimate the on-axis brilliance from the radiated flux in the central cone as discussed below. (In this document, we used an accurate numerical computation based on the Bessel function approximation for the tuning curve calculations.) The on-axis brilliance $B_{0}$ may be approximated by the photon flux in the central cone $F_{\text {cone }}$ divided by the phase-space area $(2 \pi)^{2} \Sigma_{x} \Sigma_{x^{\prime}} \Sigma_{y} \Sigma_{y^{\prime}}$ occupied by the photon beam [13]. Three limiting cases may be defined that depend on the beam emittance vs. the diffraction-limited photon-beam size $\sigma_{r}$ and divergence $\sigma_{r^{\prime}}$ : cases A-C below.

In the emittance dominated regime (case A), the smaller the emittance, the higher the brilliance. In the intermediate regime $(\mathrm{B} 1, \mathrm{~B} 2)$, where the contribution from $\sigma_{r}$, to the phase space is important or dominant, making the $\beta$ function small will increase the brilliance. For the APS (case B1), a reduction of $\beta_{y}$ does not increase the divergence of

the emitted radiation significantly, but the photon-beam size will be smaller by $\sqrt{\beta_{y}}$, and, thus the brilliance will increase by the same factor.

The scaling of brilliance vs. the number of undulator periods $N$ is also indicated. (The flux in the central cone scales linearly with $N$ and the current $I$. The natural divergence $\sigma_{r^{\prime}}$ scales as $1 / \sqrt{N}$.) At very high harmonic energies, the beam energy spread and the undulator magnetic field errors smear the harmonics and make the undulator behave wiggler-like, and the brilliance will scale almost linearly with $N$. 
The on-axis brilliance $B_{0}$ can be divided into the following regimes:

A. Emittance dominated regime: $\sigma_{x}, \sigma_{y} \gg \sigma_{r}$ and $\sigma_{x^{\prime}}, \sigma_{y^{\prime}} \gg \sigma_{r^{\prime}}$

$$
B_{0} \sim \frac{F_{\text {cone }}}{(2 \pi)^{2} \varepsilon_{x} \varepsilon_{y}}, B_{0} \sim N
$$

B1. Intermediate regime 1: $\sigma_{x}, \sigma_{y}>>\sigma_{r}$ and $\sigma_{x^{\prime}}>>\sigma_{r^{\prime}}, \sigma_{y^{\prime}}<<\sigma_{r^{\prime}}$ (important for the APS at low-to-intermediate energies)

$$
B_{0} \sim \frac{F_{\text {cone }}}{(2 \pi)^{2} \varepsilon_{x} \sigma_{y} \sigma_{r^{\prime}}}, B_{0} \sim N^{1.5}
$$

B2. Intermediate regime 2: $\sigma_{x}, \sigma_{y}>>\sigma_{r}$ and $\sigma_{x^{\prime}}, \sigma_{y^{\prime}}<<\sigma_{r^{\prime}}$

$$
B_{0} \sim \frac{F_{\text {cone }}}{(2 \pi)^{2} \sigma_{x} \sigma_{y} \sigma_{r^{\prime}}^{2}}, B_{0} \sim N^{2}
$$

C. Diffraction-limited regime: $\sigma_{x}, \sigma_{y}<<\sigma_{r}$ and $\sigma_{x^{\prime}}, \sigma_{y^{\prime}}<<\sigma_{r^{\prime}}$

$$
B_{0} \sim \frac{F_{\text {cone }}}{(2 \pi)^{2} \sigma_{r}^{2} \sigma_{r^{\prime}}^{2}}=\frac{F_{\text {cone }}}{(\lambda / 2)^{2}}, B_{0} \sim N
$$

\section{APS Storage Ring Lattices}

There are several storage ring lattices used in the calculations in this document. We will start with the APS design lattice as it was described in Technical Bulletin ANL/APS/TB-17 [2]. Then we will describe today's two operational lattices, the "lowemittance" and "high-emittance" lattices, and finally we will look into the future for lattices with even smaller coupling - approaching 0.1\%. The papers by Emery et al. give a good overview of the recent APS lattice improvements from the APS design stage to today (including top-up operations) $[16,17]$. 
In the calculations, we use the minimum values of the $\beta$ functions corresponding to an upright phase space ellipse (nominally at the center $s_{o}$ of the straight sections) for the model lattices listed in Tables A1 - A3. The IDs are located off-center $L_{\text {offset }}$ by about $1.3 \mathrm{~m}$, however, the effective change of the source position $L_{\text {eff }}$ from the location $s_{o}$ will be less due to the finite electron-beam divergence as explained in Technical Bulletin ANL/APS/TB-33 [9]. (The formula given in the earlier technical bulletin is corrected here.) In the $x$ direction, where the electron-beam divergence is much larger then the natural divergence, the offset can be ignored. In the y direction, the effective offset in source position $L_{\text {eff }}$ should be used instead. It is given by

$$
L_{e f f}=\frac{L_{o f f s e t}}{1+\left(\sigma_{y^{\prime}} / \sigma_{r^{\prime}}\right)^{2}} .
$$

Inserting realistic values for the divergences $\left(\sigma_{r^{\prime}} \sim 6 \mu \mathrm{rad}\right.$ at $8 \mathrm{keV}$ and $\sigma_{y^{\prime}} \sim 3$ $\mu \mathrm{rad}$ for the low-emittance lattice), we get $L_{\text {eff }} \sim 1.0 \mathrm{~m}$. This value however, should be compared to the typical distance from the source $D \sim 30 \mathrm{~m}$, where there is a limiting aperture that defines the angular acceptance of the photon beam. Hence, the change in the measured on-axis angular flux density $2 \Delta D / D$ will be $\sim 7 \%$, and the offset can typically be ignored also in the y direction. There is also an uncertainty in the location of $s_{o}$ itself that is comparable to $L_{e f f}$. The location $s_{o}$ is designed to be at the center of the straight sections, but variations of $0.9 \mathrm{~m} \mathrm{rms}$ for the horizontal direction and $0.4 \mathrm{~m} \mathrm{rms}$ for the vertical direction may occur from one sector to the next [18].

The calculated lattice parameters agree rather well with recent measurements of the sector-averaged $\beta_{x, y}\left(s_{o}\right)$ for the low-emittance lattice. The model was accurate to within $5 \%$ for $\beta_{x}\left(s_{o}\right)$ and $20 \%$ for $\beta_{y}\left(s_{o}\right)$ [18]. There are constant efforts to improve the optics to yield still better agreement in the future.

\section{Design Lattice}

The original APS design lattice had a natural emittance $\varepsilon$ of $8.2 \mathrm{~nm}$-rad with a minimum coupling specification of $10 \%$ and zero dispersion in the 5-m-long straight sections for the IDs. The storage ring energy was $7.0 \mathrm{GeV}$, the beam current was 100 $\mathrm{mA}$, and the $\beta$ functions were $14 \mathrm{~m}$ and $10 \mathrm{~m}$ in the horizontal and vertical direction, respectively. Table A1 (reproduced from ANL/APS/TB-17 [2]) shows the relevant parameters for the design lattice. In this note, we refer to this lattice as the design lattice. We ran with this lattice from the start of commissioning to December of 1997. 
Table A1: The APS $8.2 \mathrm{~nm}$-rad design lattice and source parameters at the center of the ID straight sections.

\begin{tabular}{ll}
\hline Parameter ${ }^{1)}$ & Value \\
\hline Storage ring energy, $E$ & $7.0 \mathrm{GeV}$ \\
Storage ring current, $I$ & $100 \mathrm{~mA}$ \\
Natural emittance, $\varepsilon$ & $8.2 \times 10^{-9} \mathrm{~m}-\mathrm{rad}$ \\
Horizontal emittance, $\boldsymbol{\varepsilon}_{x}$ & $7.45 \times 10^{-9} \mathrm{~m}-\mathrm{rad}$ \\
Vertical emittance, $\mathcal{E}_{y}$ & $7.45 \times 10^{-10} \mathrm{~m}-\mathrm{rad}$ \\
Minimum coupling specification & $10 \%$ \\
Horizontal beta function, $\beta_{x}$ & $14.2 \mathrm{~m}$ \\
Vertical beta function, $\beta_{y}$ & $10.0 \mathrm{~m}$ \\
Horizontal beam size, $\sigma_{x}$ & $325 \mu \mathrm{m}$ \\
Vertical beam size, $\sigma_{y}$ & $86 \mu \mathrm{m}$ \\
Horizontal beam divergence, $\sigma_{x}$, & $23 \mu \mathrm{rad}$ \\
Vertical beam divergence, $\sigma_{y}$, & $9 \mu \mathrm{rad}$ \\
& \\
\hline
\end{tabular}

1) The dispersions $\eta_{x}, \eta_{x}$, and the alpha functions $\alpha_{x}, \alpha_{y}$ are zero. The beam energy spread $\delta E / E$ is $0.096 \%$.

\section{Present Lattices}

There are two operational lattices used today, and both are listed for completeness. Both are low- $\beta_{y}$ lattices, i.e., a $\beta_{y}$ of $\sim 4 \mathrm{~m}$ is used in all ID straight sections, reducing the vertical beam size and improving instability thresholds. They have been in operation since March of 1998. (A hybrid lattice, where only one straight section had $\beta_{y} 4 \mathrm{~m}$, and the others had $10 \mathrm{~m}$, was used for a short time between January 1998 and March 1998.) The low- $\beta_{y}$ lattice (high-emittance lattice) has an equilibrium emittance of $7.7 \mathrm{~nm}-\mathrm{rad}$, and was the standard lattice from March 1998 until September 2001. In October 2001, we adopted a low-emittance version of the low- $\beta_{y}$ lattice as the standard operation lattice, which we call the low-emittance lattice. The old low- $\beta_{y}$ lattice is now called the high-emittance lattice, and it is used occasionally for one-week periods during non-top-up runs. (The high-emittance lattice was not used for calculations in this document.)

The horizontal emittance for the APS low-emittance lattice $\varepsilon_{x}$ is $3.5 \mathrm{~nm}$-rad, and it has been reduced by a factor of two in comparison with the $8.2 \mathrm{~nm}$-rad design lattice. Further, the coupling was reduced by a factor of ten to $1 \%$. The smaller emittance could only be achieved by introducing nonzero dispersion in the straight sections, however. In comparison, the high-emittance lattice allows zero dispersion in the straight sections, the same coupling, but slightly changed beta functions. Table A2 compares the important parameters for the two lattices. 
Table A2: Comparison of the present $3.5 \mathrm{~nm}$-rad low-emittance and the $7.7 \mathrm{~nm}$-rad highemittance lattices and source parameters at the center of the ID straight sections.

\begin{tabular}{lll}
\hline Parameter $^{1)}$ & Low-Emittance & High-Emittance \\
\hline & & \\
Storage ring energy, $E$ & $7.0 \mathrm{GeV}$ & $7.0 \mathrm{GeV}$ \\
Storage ring current, $I$ & $100 \mathrm{~mA}$ & $100 \mathrm{~mA}$ \\
Beam energy spread, $\delta E / E$ & $0.096 \%$ & $0.096 \%$ \\
Horizontal emittance, ${ }^{2)} \varepsilon_{x}$ & $3.5 \times 10^{-9} \mathrm{~m}-\mathrm{rad}$ & $7.7 \times 10^{-9} \mathrm{~m}-\mathrm{rad}$ \\
Vertical emittance, $\varepsilon_{y}$ & $3.5 \times 10^{-11} \mathrm{~m}-\mathrm{rad}$ & $7.7 \times 10^{-11} \mathrm{~m}-\mathrm{rad}$ \\
Coupling constant & $1 \%$ & $1 \%$ \\
Horizontal beta function, $\beta_{x}$ & $14.4 \mathrm{~m}$ & $16.1 \mathrm{~m}$ \\
Vertical beta function, $\beta_{y}$ & $4.0 \mathrm{~m}$ & $4.3 \mathrm{~m}$ \\
Dispersion function, $\eta_{x}$ & $0.124 \mathrm{~m}$ & $0.0 \mathrm{~m}$ \\
Horizontal beam size, $\sigma_{x}$ & $254 \mu \mathrm{m}$ & $351 \mu \mathrm{m}$ \\
Vertical beam size, $\sigma_{y}$ & $12 \mu \mathrm{m}$ & $18 \mu \mathrm{m}$ \\
Horizontal beam divergence, $\sigma_{x}$, & $15.6 \mu \mathrm{rad}$ & $21.8 \mu \mathrm{rad}$ \\
Vertical beam divergence, $\sigma_{y}$, & $3.0 \mu \mathrm{rad}$ & $4.2 \mu \mathrm{rad}$ \\
& & \\
\hline
\end{tabular}

1) The dispersion function $\eta_{x}$, is zero, and the alpha functions $\alpha_{x}, \alpha_{y}$ are zero. The horizontal source size includes the effect of the dispersion function $\eta_{x}$.

2) The emittance is derived from model calculations. During operations, when many ID gaps are lowered at the same time, the additional synchrotron radiation loss causes the equilibrium emittance to be slightly smaller-maximum reduction will be $10 \%$. We have chosen to ignore this effect in order to give a conservative value for the calculated on-axis brilliances.

\section{Future Lattices}

There is a possibility in the future that the coupling can be made much smaller than the $1 \%$ we use today. The ultimate limit would technically be around $0.1 \%$. Further, one can also envision increasing the beam current up to $300 \mathrm{~mA}$, which is technically feasible for the storage-ring components (but the radiated power-on-axis power density and total power-onto the front-end components does not allow this at the present time).

The possibility of making $\beta_{x}$ different in specific sectors to suit particular needs of users exists, but at the expense of making it more difficult to achieve the lowest emittance possible.

It is also technically feasible to introduce longer straight sections $(10.7 \mathrm{~m})$ at the expense of a slightly increased emittance $[17,19]$. We have also made brilliance calculations for this case (for an optimized period length of $2.5 \mathrm{~cm}$ ) that will represent the 
ultimate technical limitation of the APS storage ring. The parameters used for calculations of on-axis brilliances for both lattices are listed in Table A3.

Table A3: Comparison of future high-current, low-coupling, low-emittance lattices.

A $3.5 \mathrm{~nm}$-rad lattice vs. $4.0 \mathrm{~nm}$-rad lattice with $10.7 \mathrm{~m}$ long straight sections.

\begin{tabular}{lll}
\hline Parameter $^{1)}$ & $3.5 \mathrm{~nm}-\mathrm{rad}$ lattice & $4.0 \mathrm{~nm}-\mathrm{rad}$ lattice \\
\hline & & \\
Storage ring energy, $E$ & $7.0 \mathrm{GeV}$ & $7.0 \mathrm{GeV}$ \\
Storage ring current, $I$ & $300 \mathrm{~mA}$ & $300 \mathrm{~mA}$ \\
Beam energy spread, $\delta E / E$ & $0.096 \%$ & $0.097 \%$ \\
Horizontal emittance, ${ }^{2)} \varepsilon_{x}$ & $3.5 \times 10^{-9} \mathrm{~m}-\mathrm{rad}$ & $4.0 \times 10^{-9} \mathrm{~m}-\mathrm{rad}$ \\
Vertical emittance, $\varepsilon_{y}$ & $3.5 \times 10^{-12} \mathrm{~m}-\mathrm{rad}$ & $4.0 \times 10^{-12} \mathrm{~m}-\mathrm{rad}$ \\
Coupling constant & $0.1 \%$ & $0.1 \%$ \\
Horizontal beta function, $\beta_{x}$ & $14.9 \mathrm{~m}$ & $20.0 \mathrm{~m}$ \\
Vertical beta function, $\beta_{y}$ & $3.7 \mathrm{~m}$ & $5.3 \mathrm{~m}$ \\
Dispersion function, $\eta_{x}$ & $0.120 \mathrm{~m}$ & $0.052 \mathrm{~m}$ \\
Horizontal beam size, $\sigma_{x}$ & $255 \mu \mathrm{m}$ & $287 \mu \mathrm{m}$ \\
Vertical beam size, $\sigma_{y}$ & $4 \mu \mathrm{m}$ & $5 \mu \mathrm{m}$ \\
Horizontal beam divergence, $\sigma_{x}$, & $15.3 \mu \mathrm{rad}$ & $14.1 \mu \mathrm{rad}$ \\
Vertical beam divergence, $\sigma_{y}$, & $1.0 \mu \mathrm{rad}$ & $0.9 \mu \mathrm{rad}$ \\
Length of straight section, $\mathrm{L}$ & $5.0 \mathrm{~m}$ & $10.7 \mathrm{~m}$ \\
& & \\
\hline
\end{tabular}

1) The dispersion function $\eta_{x}$, is zero. The horizontal source size includes the effect of the dispersion function $\eta_{x}$. The $\alpha$ functions $\alpha_{x}, \alpha_{y}$ are zero.

${ }^{2)}$ The emittance is derived from model calculations.

\section{Bunch Pattern and Timing Structure}

The storage ring is operating in either single-fill mode or hybrid-fill mode. The actual bunch pattern is not needed for the calculations in this document, as we only need the average beam current. However, it is necessary to know the peak current for calculations of peak quantities such as the on-axis peak brilliance. This is generally of interest when comparing quantities for the APS vs. fourth-generation light sources, where the on-axis peak brilliance is the most-often quoted quantity. The peak current for any bunch pattern (Gaussian-shaped bunch) is related to the average beam current per bunch, $I_{\text {ave }}$ by

$$
I_{\text {peak }}=I_{\text {ave }} \frac{3683}{\sqrt{2 \pi} \sigma_{\text {bunch }}(n s)}
$$


where $\sigma_{\text {bunch }}$ is the rms bunch length in ns. (The peak current/bunch is $177 \mathrm{~A}$ for the standard singlets fill pattern; thus to get the on-axis peak brilliance from the calculated average on-axis brilliance at $100 \mathrm{~mA}$, multiply the value by 1770.)

One or more long trains of bunches (15 ns long) are accelerated in the linac to 325 $\mathrm{MeV}$ from the gun into the positron accumulator ring. The bunches are damped in transverse and longitudinal coordinates and accumulated one after the other until extraction is triggered, which occurs every 0.5 seconds. The bunch length at extraction is about $1 \mathrm{~ns}$. The extracted beam is injected into a booster that ramps the single bunch from $325 \mathrm{MeV}$ to $7 \mathrm{GeV}$ in about $225 \mathrm{~ms}$. At the end of the booster cycle, the bunch is extracted and injected into the storage ring. The bunch can be injected into any of the 1296 buckets that are spaced by $2.842 \mathrm{~ns}$, forming any arbitrary pattern after several injection cycles.

The storage ring rf frequency of $351.927 \mathrm{MHz}$ determines the bucket spacing of $2.842 \mathrm{~ns}$. The circumference of $1104 \mathrm{~m}$ gives a revolution frequency of $271.5 \mathrm{kHz}$, which allows 1296 buckets in 3683 ns.

The storage ring is filled with either the singlets bunch pattern or the hybrid bunch pattern. The singlets bunch pattern has a lower lifetime than patterns with less charge per bunch due to natural internal scattering processes. The singlets are therefore compatible with top-up operation where a low lifetime ( $7 \mathrm{hrs}$ ) is not a major drawback. For non-top-up operation, we use the hybrid pattern and optionally the high-emittance lattice to increase the lifetime and time between injections.

Top-up is actually necessary for running the low-emittance lattice given the lifetime of 7 hours. The hybrid pattern can be used for low- and high-emittance lattices. The lifetime requirement for using a lattice and bunch pattern in a regular 12-hour fill operation is 20 hours. For a 24-hour fill, the lifetime requirement is 30 hours.

The standard singlets bunch pattern is $102 \mathrm{~mA}$ of average current in a train of 23 bunches, (4.43 mA/bunch or $16 \mathrm{nC} /$ bunch), each spaced by $153 \mathrm{~ns}$ (54 buckets). There is a gap of $306 \mathrm{~ns}$ (108 buckets). This pattern is called the singlets bunch pattern. The rms bunch length for this charge per bunch is $36 \mathrm{ps,} \mathrm{corresponding} \mathrm{to} 177$ A peak current.

The hybrid bunch pattern is $102 \mathrm{~mA}$ in one bunch of $5 \mathrm{~mA}$ plus 8 groups of 7 consecutive bunches $(1.73 \mathrm{~mA} /$ bunch or $6.3 \mathrm{nC} / \mathrm{bunch})$ spaced by 24 buckets ( $68 \mathrm{~ns})$. These 8 groups are opposite the $5 \mathrm{~mA}$ bunch, allowing a $1.5 \mu \mathrm{sec}$ gap on both sides. A variation that has been used once is filling $15 \mathrm{~mA}$ in 3 consecutive bunches and the rest in the 8 groups of 7 bunches. 


\section{Appendix B: Additional Undulator A Magnetic Properties}

Different tuning techniques were used to tune for small phase errors, the first- and second field integrals, and the integrated multipole moments for all possible gap regions. Most of the tuning was done with 0.1-0.2-mm-thick magnetic material shims (usually soft iron). These shims are located on top of the magnets. The precise location of such shims depends on the explicit parameter that needs to be corrected. Some other types of shims invented at the APS were used as well, i.e., side shims attached to the outbound side of a pole reducing the magnetic field. Pure shims that correct only one property of the device and do not affect others were found and used, especially for the phase tuning (i.e., pure phase shims or pure trajectory shims) $[10,20]$.

The plan to use two undulators in tandem in one straight section of the storage ring will require phasing of the devices. Our experience with phasing of the devices for the APS free-electron-laser project will be very useful in this case [21].

A large variety of magnetic sensors has been used to measure different magnetic properties of the insertion devices. The first six figures below (Figs. B1 - B6) show data that were derived from Hall probe measurements, and the rest (Figs. B7 - B11) show data from coil measurements for integrated properties. The earth field was subtracted for the coil data but not for the Hall probe data.

\section{Hall Probe Measurements}

Figure B1 shows an example of a calculated electron trajectory and the periodaveraged electron trajectory. The entrance and exit angles for the electron are also marked. (The entrance angle is calculated in the analysis code to make the periodaveraged trajectory angle zero when averaged over the regular part of the undulator.) Note that tuning of IDs for the storage ring emphasizes different aspects than tuning of devices for a free-electron laser. For example, tuning for small phase errors is important for storage ring IDs whereas tuning for trajectory straightness and phase matching is important for free-electron-laser magnetic devices.

The trajectory straightness and trajectory angle for the period-averaged electron trajectory calculated from 23 measured IDs are shown vs. gap in Figures B2 and B3, respectively. 


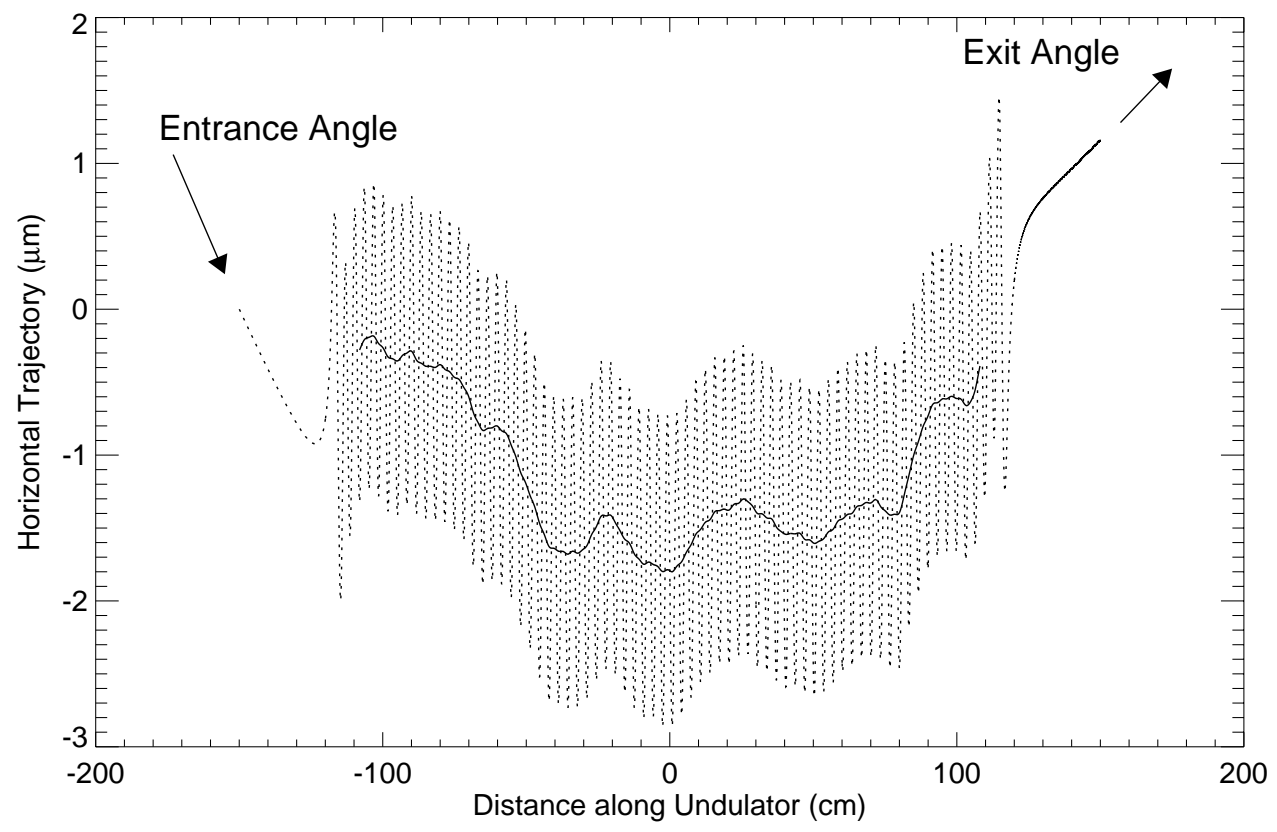

Figure B1. Example of calculated horizontal electron trajectory from magnetic field measurements at $10.5 \mathrm{~mm}$ gap ( $K_{\text {eff }}$ is 2.750 ) at $7.0 \mathrm{GeV}$ (dotted curve). The periodaveraged trajectory is shown in the regular part of the undulator (solid curve). The regular part excludes 5 end-poles at each end of the undulator. The entrance angle $(-4.2 \mu \mathrm{rad})$ and exit angle $(2.1 \mu \mathrm{rad})$ are defined for clarity. The electron was given this kick at the entrance of the undulator to make the period-averaged trajectory angle zero when averaged over the regular part-this corresponds to the case when $\theta=0$ and it defines the optimum view angle. 


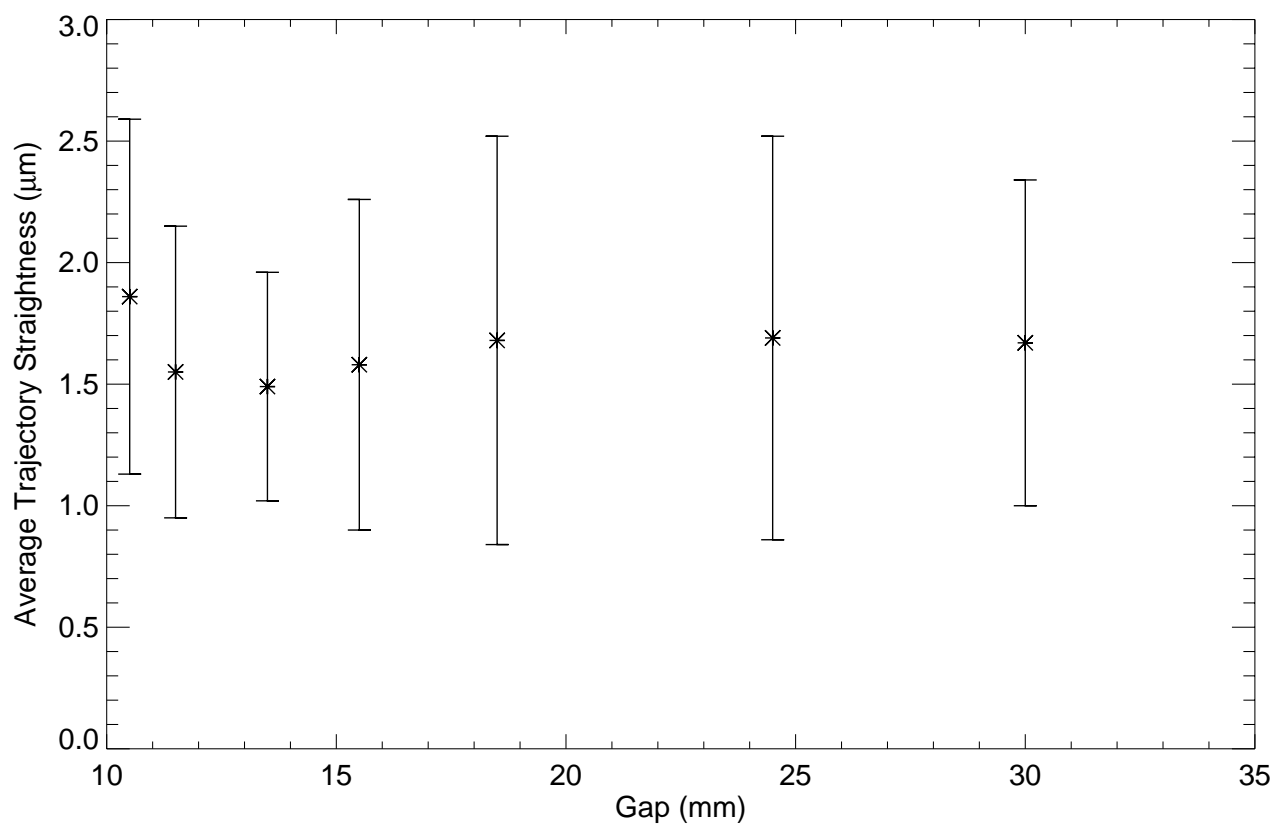

Figure B2. Trajectory straightness in the horizontal plane vs. gap at beam energy 7.0 GeV. The error bars show the rms variation over 23 measured IDs. The trajectory straightness is defined as the maximum difference in position for the period-averaged trajectory within the regular part of the undulator. (The period-averaged trajectory shown in Figure B1 at $10.5 \mathrm{~mm}$ gap falls near the average of $2 \mu \mathrm{m}$.) The trajectory straightness is $\sim 2 \mu \mathrm{m}$ at all gaps.

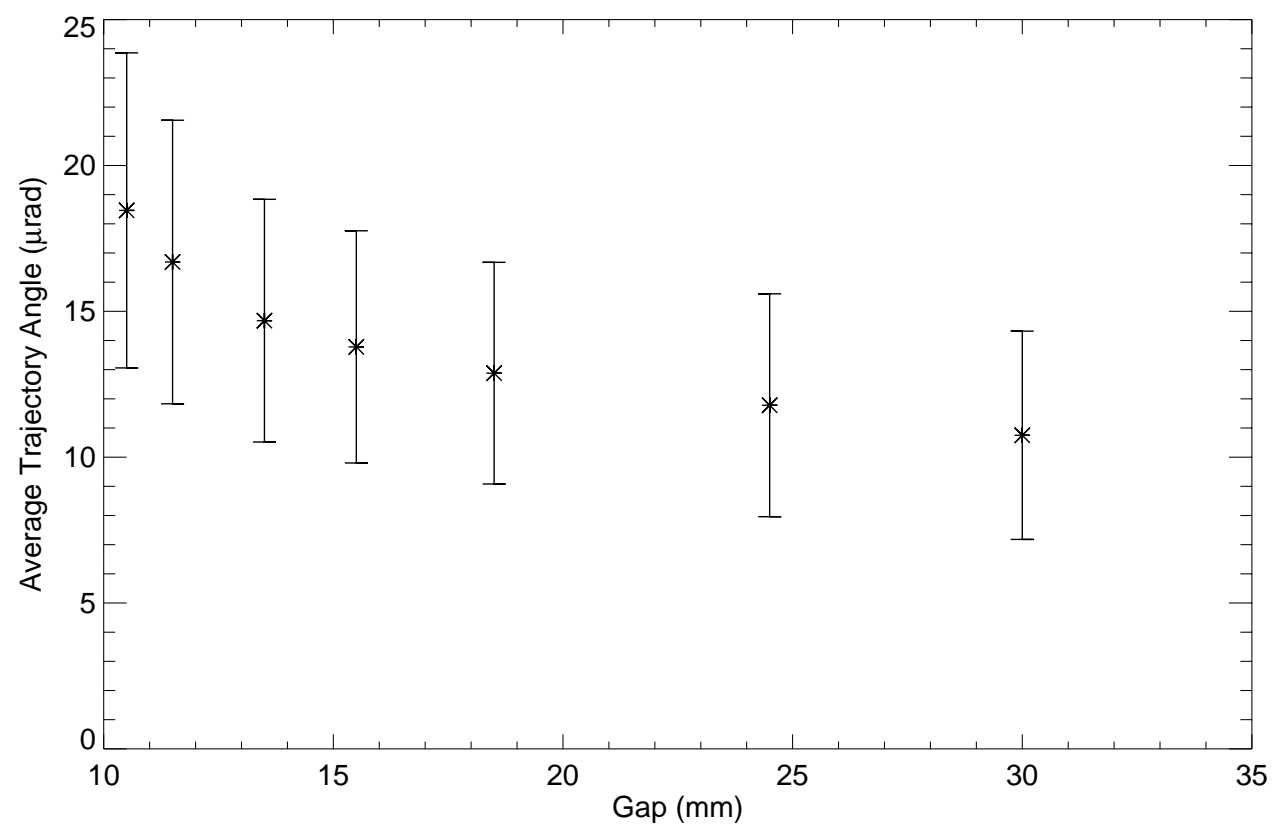

Figure B3. Trajectory angle in the horizontal plane vs. gap at beam energy 7.0 GeV. The error bars show the rms variation over 23 measured IDs. The trajectory angle is defined as the maximum difference in angle for the period-averaged trajectory within the regular part of the undulator. There is a small dependency on the gap. 
As user demand for better beam stability is increasing, it will become important to tune the IDs to smaller values for the first and second field integrals. For this purpose, we have also started to record both the entrance and exit angle vs. gap (the difference between the exit angle and the entrance angle from the Hall probe measurements equals the first field integral from coil measurements.) We give examples of the measured entrance and exit angles vs. gap in Figure B4.

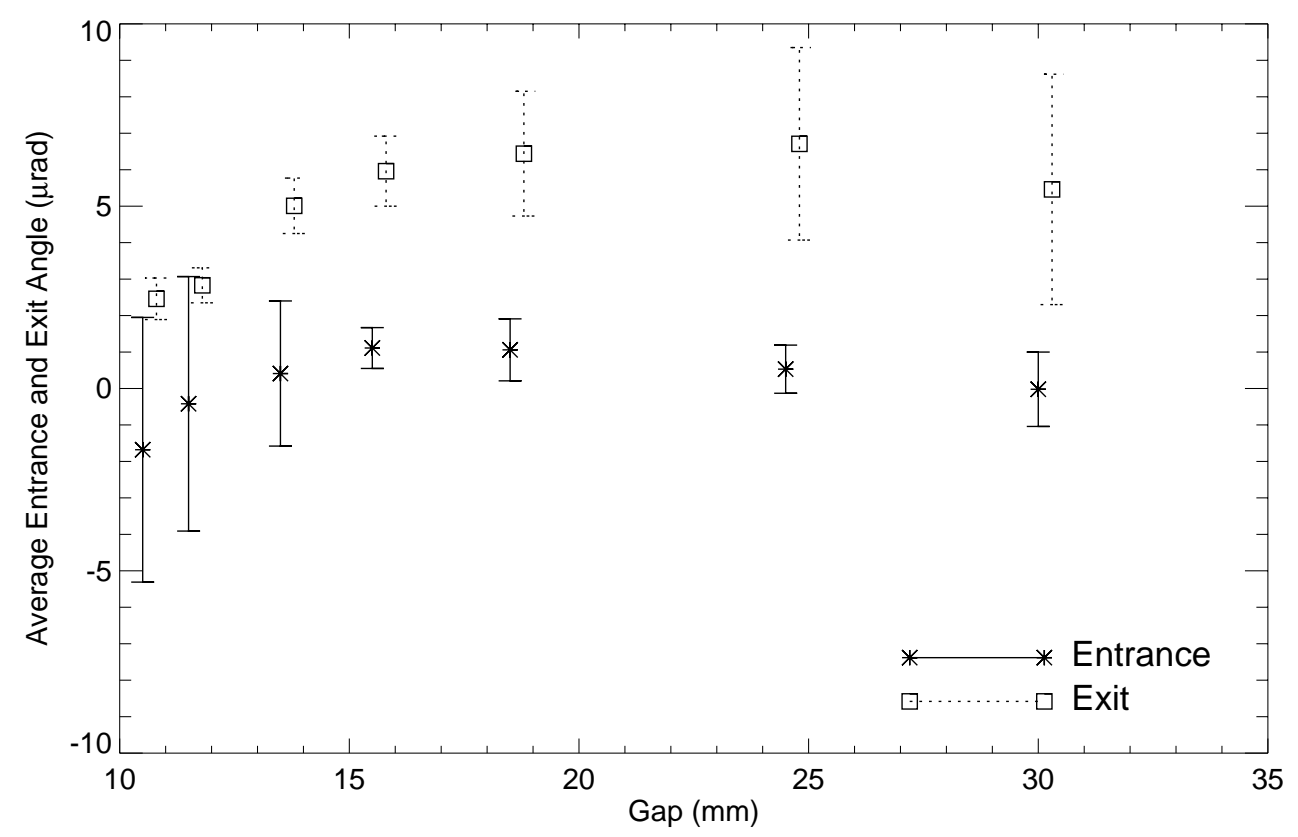

Figure B4. Measured entrance and exit angles in the horizontal plane vs. gap. (Note, only 2 IDs were included because this data was not calculated initially; the data points and rms variations should be used as guidance only for future work to reduce gap-dependent steering of the electron beam.) The angles were defined in Figure B1.

Both the rms phase error and the rms pole-to-pole peak magnetic field variation were vendor specifications (at a gap of $11.5 \mathrm{~mm}$ ). The measured gap dependencies of these parameters are shown in Figures B5 and B6, respectively. 


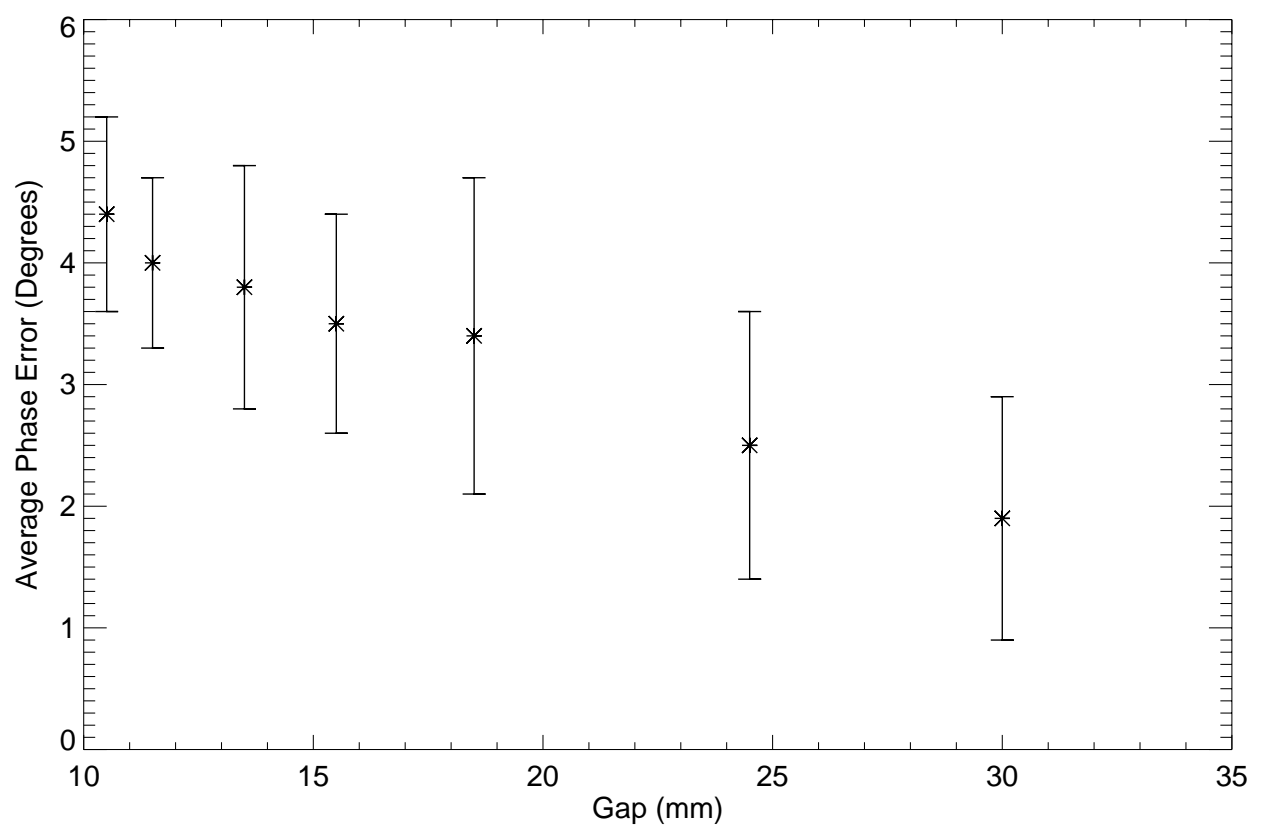

Figure B5. Measured rms phase error vs. gap. The error bars show the rms variation over 23 measured IDs. Note that the specification to the vendor was $8^{\circ}$ rms variation at 11.5 $\mathrm{mm}$ gap. This was met by a very large margin. (The measured average rms phase error is only $4^{\circ}$ ). The rms phase error decreases with increasing gap and approaches $2^{\circ}$ for large gaps.

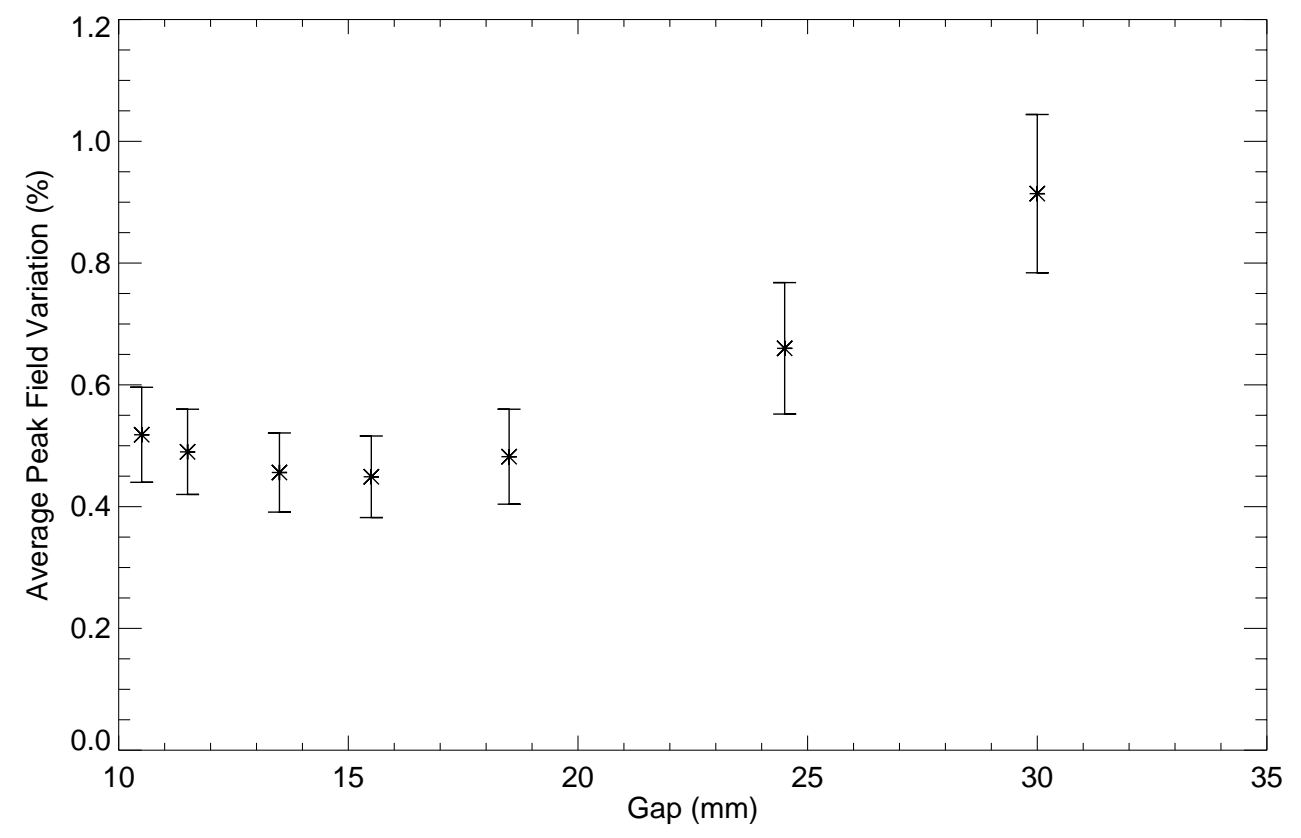

Figure B6. Measured rms pole-to-pole peak magnetic field variation vs. gap. The error bars show the rms variation over 23 measured IDs. On the average, the specified value to the vendor to be less than $0.5 \%$ at $11.5 \mathrm{~mm}$ was met (not an important design parameter). 
The phase error indicates the deviation from perfect matching in phase between the electron and the emitted radiation from pole to pole. The slippage between the electron and the light is exactly one period of the emitted radiation $(\lambda)$ when one period of the undulator $\left(\lambda_{u}\right)$ has been traversed for an ideal sinusoidal magnetic field, i.e., the electron falls behind the light by one period $(\lambda)$. A phase error of \pm 1 degree, for instance, indicates that there is an offset by $\pm \lambda / 360$ from one period to the next. The phase errors are calculated at each pole (slippage is $\lambda / 2$ from pole to pole for a perfect device) in the regular part of the undulator (omitting 5 end-poles at each end).

The slippage $\Delta S$ from $z_{1}$ to $z_{2}$ is

$$
S\left(z_{2}\right)-S\left(z_{1}\right)=\int_{z_{1}}^{z_{2}}\left(\frac{1}{2 \gamma^{2}}+\frac{1}{2} x^{\prime 2}(z)\right) d z
$$

where $x^{\prime}(z)$ is the electron's angle in the horizontal plane, which is calculated from the first field integral $J_{1 y}(z)$ of the measured vertical magnetic field $B_{y}(z)$

$$
x^{\prime}(z) \sim\left(-\frac{e}{2 m_{e} c}\right) J_{1 y}(z), J_{1 y}(z)=\int_{-\infty}^{z} B_{y}\left(z^{\prime}\right) d z^{\prime} .
$$

where $e$ is the electron charge, $m_{e}$ is the electron rest mass, $\gamma$ is the relativistic factor for the electron, and $c$ is the speed of light.

A linear fit $(a+b z)$ is made to the slippage $\Delta S$. The effective $K$ value $K_{\text {eff }}$ is then calculated from the slope $b$

$$
1+K_{\text {eff }}^{2} / 2=b,
$$

and hence the on-axis radiated wavelength $\lambda_{1}(0)=\frac{\lambda_{u}}{2 \gamma^{2}}\left(1+K_{\text {eff }}^{2} / 2\right)$ will be known.

[The effective magnetic field $B_{\text {eff }}$ is calculated from the relation $K_{\text {eff }}=0.934 \lambda_{u}(\mathrm{~cm}) B_{\text {eff }}(T)$.]

Once $\lambda_{1}(0)$ is known, the phase errors $P_{i}$ are obtained from the deviation of phases $P\left(z_{i}\right)$ at the poles $i$

$$
P_{i}=\left(P\left(z_{i}\right)-i / 2\right) * 360, P\left(z_{i}\right)=S\left(z_{i}\right) / \lambda_{1}(0) .
$$

The rms variation (over 134 regular poles) of the phase error vs. gap was shown in Figure B5 above. 


\section{Coil Measurements}

The Undulator A magnetic properties that are important for storage ring operation are listed in Table B1 along with the measured values. In all cases, the devices have been tuned to meet these requirements. In addition to the values listed in the table, graphs are shown below that give the maximum change in the field integrals for each insertion device (Figures B7 -B8) and the gap dependency of the integrated multipole moments (Figures B9-B11).

Table B1: Undulator A specifications and measurements of beam-orbit-related parameters.

\begin{tabular}{|c|c|c|}
\hline Parameter & Specified Value & Measured Value \\
\hline $\begin{array}{l}\text { Maximum change in the first field } \\
\text { integral, } \Delta J_{1 h} \text { (horizontal field) }{ }^{1,2)}\end{array}$ & $\left|\Delta J_{1 h}\right|<50$ Gauss-cm & 48 Gauss-cm \\
\hline $\begin{array}{l}\text { Maximum change in the first field } \\
\left.\text { integral, } \Delta J_{1 v} \text { (vertical field) } 1,3\right)\end{array}$ & $\left|\Delta J_{1 v}\right|<100$ Gauss-cm & 84 Gauss-cm \\
\hline $\begin{array}{l}\text { Maximum change in the second field } \\
\text { integral, } \Delta J_{2 h} \text { (horizontal field) }{ }^{1)}\end{array}$ & $\left|\Delta J_{2 h}\right|<10^{5}$ Gauss-cm ${ }^{2}$ & $0.21 \times 10^{5}$ Gauss-cm ${ }^{2}$ \\
\hline $\begin{array}{l}\text { Maximum change in the second field } \\
\left.\text { integral, } \Delta J_{2 v} \text { (vertical field }\right)\end{array}$ & $\left|\Delta J_{2 v}\right|<10^{5}$ Gauss-cm ${ }^{2}$ & $0.72 \times 10^{5}$ Gauss-cm ${ }^{2}$ \\
\hline $\begin{array}{l}\text { Quadrupole moment: normal and } \\
\text { skew 5) }\end{array}$ & $\mid$ value $\mid<50$ Gauss & $\begin{array}{l}\text { norm: }-10 \pm 23 \text { Gauss } \\
\text { skew: } 6 \pm 22 \text { Gauss }\end{array}$ \\
\hline Sextupole moment: normal ${ }^{5)}$ & |value $\mid<200$ Gauss/cm & $-40 \pm 116 \mathrm{Gauss} / \mathrm{cm}$ \\
\hline Sextupole moment: skew ${ }^{5)}$ & $\mid$ value $\mid<100$ Gauss/cm & $-8 \pm 41 \mathrm{Gauss} / \mathrm{cm}$ \\
\hline Octupole moment: normal ${ }^{5)}$ & $\mid$ value $\mid<300$ & $-33 \pm 128 \mathrm{Gauss} / \mathrm{cm}^{2}$ \\
\hline Octupole moment: skew ${ }^{5)}$ & $\mid$ value $\mid<50$ Gauss $/ \mathrm{cm}^{2}$ & $11 \pm 24$ Gauss $/ \mathrm{cm}^{2}$ \\
\hline
\end{tabular}

1) Maximum change for any of 23 measured IDs over the full measured gap range $(10.5 \mathrm{~mm}-150.0 \mathrm{~mm})$. Conversion factors at $7.0 \mathrm{GeV}: 1$ Gauss-cm $=(0.3 / 7) \mu \mathrm{rad} ; 1$ Gauss-cm ${ }^{2}=(0.3 / 7) \times 10^{-2} \mu \mathrm{m}$.

2) Additional tuning efforts using existing hardware may reduce the $\Delta J_{l h}$ to $\sim 30$ Gauss-cm.

3) Additional tuning efforts using existing hardware may reduce the $\Delta J_{l v}$ to $\sim 50$ Gauss-cm.

4) Additional tuning efforts using existing hardware may reduce the $\Delta J_{2 v}$ to $\sim 0.4 \times 10^{5}$ Gauss- $\mathrm{cm}^{2}$.

5) Average value and rms variation as measured for 23 IDs. There is a gap dependency for the higher order multipole moments, and the largest magnitudes of the average values are listed. See also graphs below that show the gap dependence on the integrated multipole moments. 


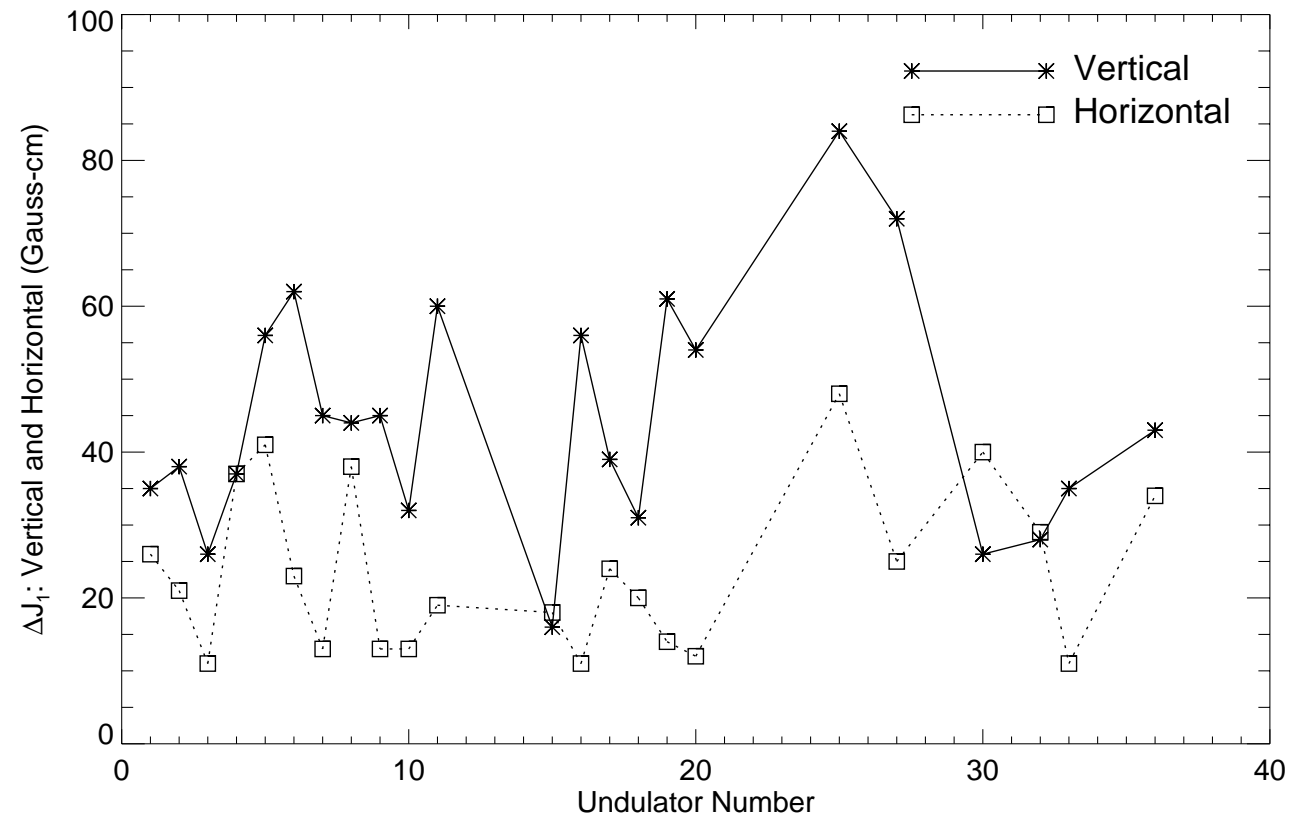

Figure B7. Measured maximum change in first field integral $\Delta J_{l}$ over the full gap range $(10.5 \mathrm{~mm}-150.0 \mathrm{~mm}$ ) vs. ID number. (Data points were connected to enhance clarity only.)

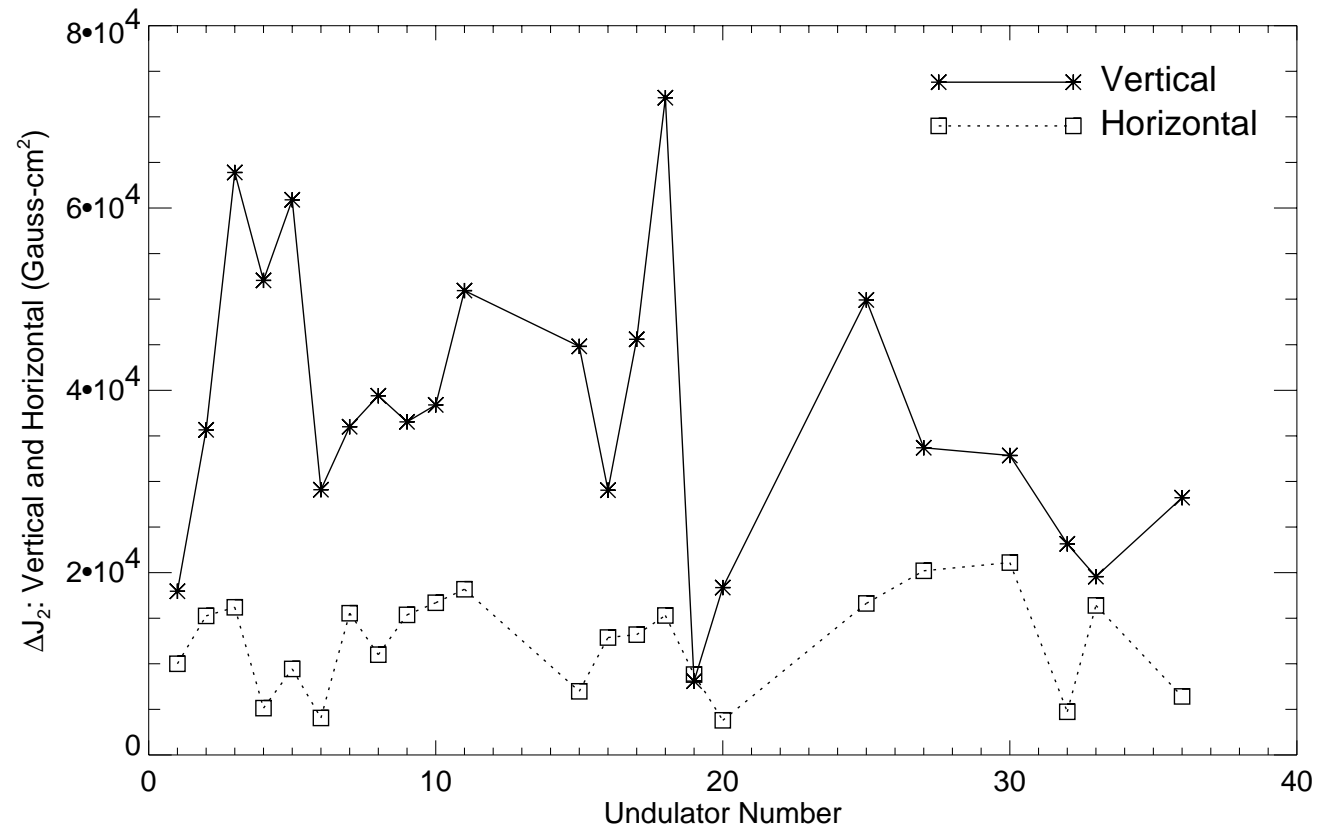

Figure B8. Measured maximum change in second field integral $\Delta J_{2}$ over the full gap range $(10.5 \mathrm{~mm}-150.0 \mathrm{~mm})$ vs. ID number. (Data points were connected to enhance clarity only.) 


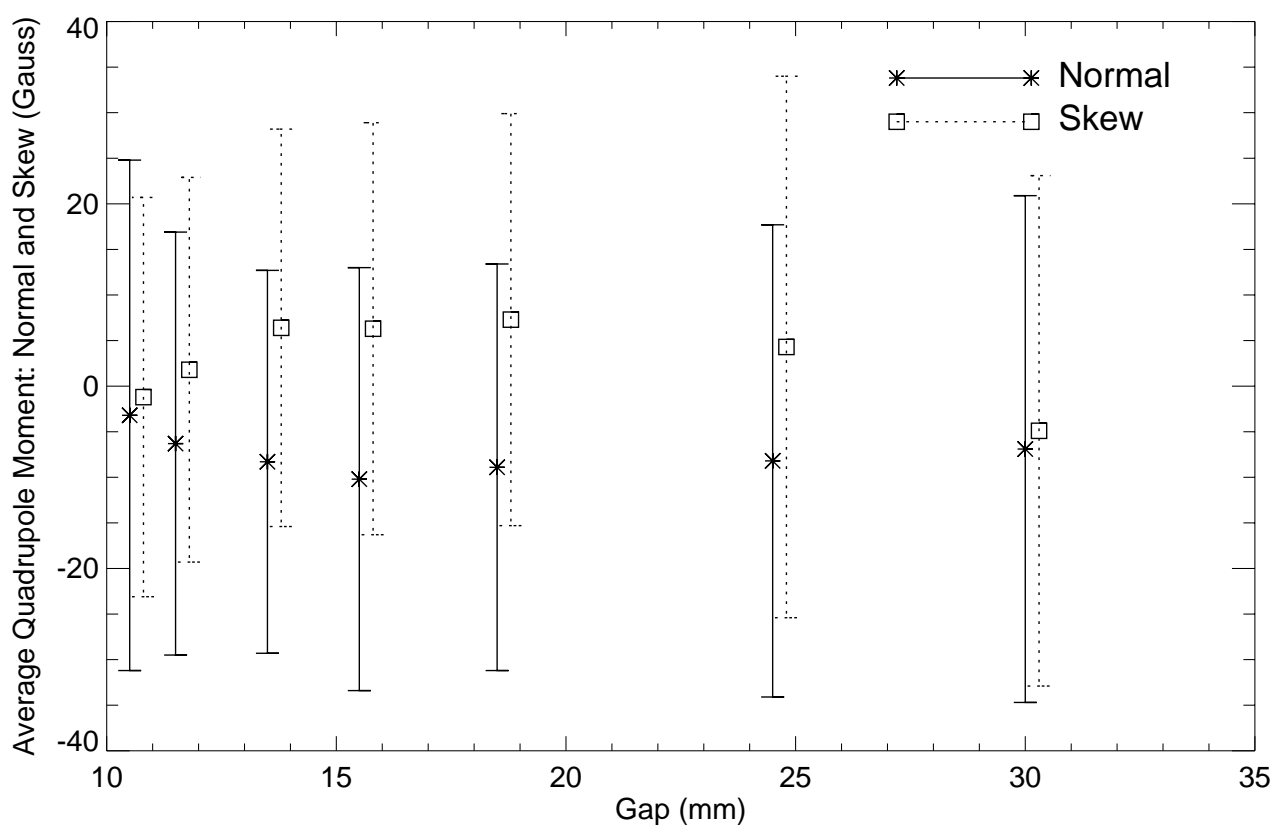

Figure B9. Average integrated normal and skew quadrupole moments vs. gap. The error bars show the rms variation over 23 measured IDs. (The skew data points were shifted by $+0.3 \mathrm{~cm}$ in gap for clarity.)

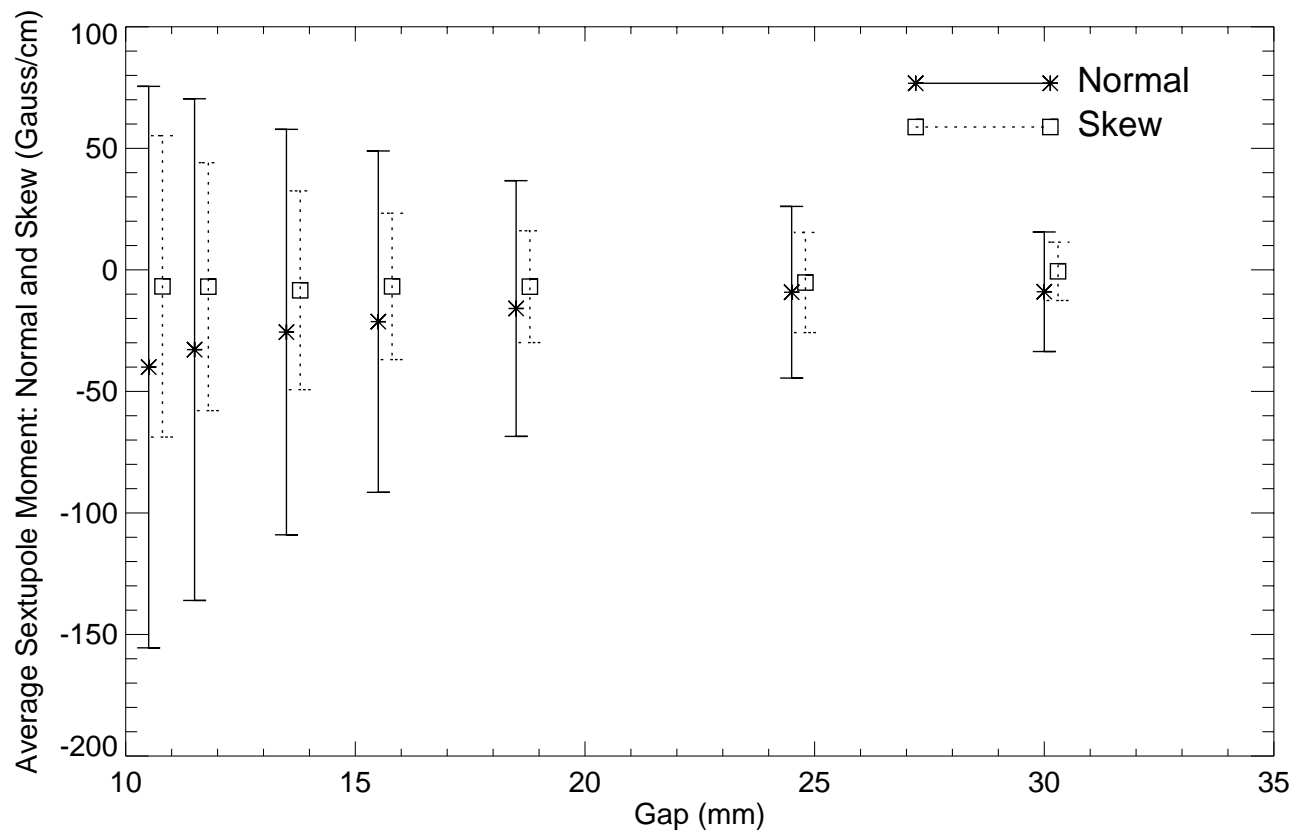

Figure B10. Average integrated normal and skew sextupole moments vs. gap. The error bars show the rms variation over 23 measured IDs. (The skew data points were shifted by $+0.3 \mathrm{~cm}$ in gap for clarity.) 


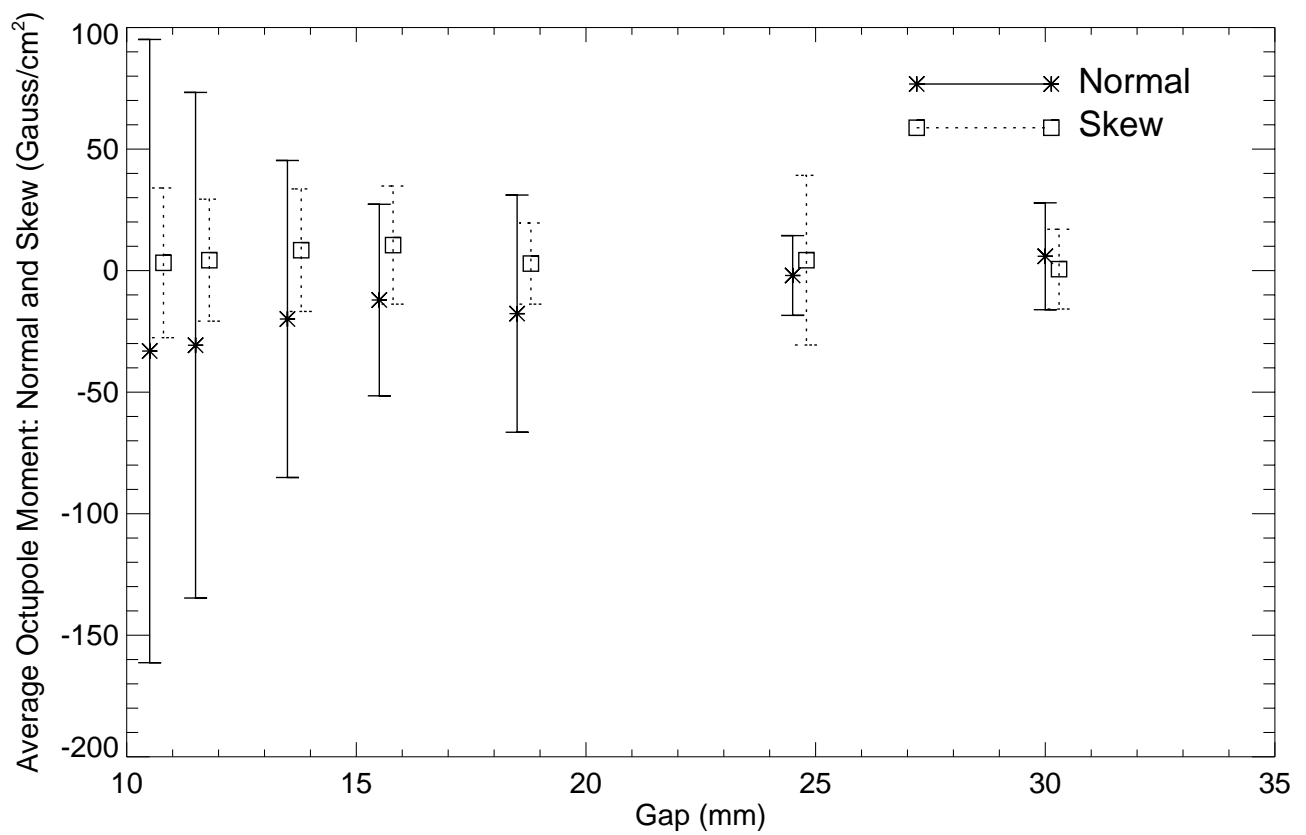

Figure B11. Average integrated normal and skew octupole moments vs. gap. The error bars show the rms variation over 23 measured IDs. (The skew data points were shifted by $+0.3 \mathrm{~cm}$ in gap for clarity.) 


\section{Appendix C: Undulator $2.7 \mathrm{~cm}$ Magnetic Properties}

In order to be able to evaluate shorter period devices for future use, we summarize the measured magnetic field vs. gap for one device that is already in use in Table $\mathrm{C} 1$.

Table C1: Measured gap dependency of the peak magnetic field $B_{\text {peak }}$ and effective field $B_{\text {eff }}$ for one 2.7-cm-period, 2.4-m-long, planar hybrid device. The effective $K$ value $K_{\text {eff }}$ and the first harmonic energy $E_{1}$ are derived from $B_{\text {eff }}$, and the powers are derived from $B_{\text {peak. }}$

\begin{tabular}{l|llllll}
\hline $\begin{array}{l}\text { Gap } \\
(\mathrm{mm})\end{array}$ & $\mathrm{B}_{\text {peak }}(\mathrm{T})^{3)}$ & $\mathrm{B}_{\text {eff }}(\mathrm{T})$ & $\mathrm{K}_{\text {eff }}$ & $\mathrm{E}_{1}(\mathrm{keV})^{5)}$ & $\begin{array}{l}\mathrm{P}_{\text {density }} \\
\left(\mathrm{kW} / \mathrm{mrad}^{2}\right)\end{array}$ & $\begin{array}{l}\mathrm{P}_{\text {total }}{ }^{6} \\
(\mathrm{~kW})\end{array}$ \\
\hline & & & & & & \\
$\mathbf{8 . 5}$ & $\mathbf{0 . 8 9 3 8}$ & $\mathbf{0 . 8 6 4 9}$ & $\mathbf{2 . 1 8 0}$ & $\mathbf{5 . 1 0 3}$ & $\mathbf{2 0 1 . 6}$ & $\mathbf{5 . 8 8}$ \\
9.0 & 0.8366 & 0.8127 & 2.049 & 5.561 & 188.4 & 5.15 \\
$\mathbf{9 . 5}$ & $\mathbf{0 . 7 8 3 0}$ & $\mathbf{0 . 7 6 3 7}$ & $\mathbf{1 . 9 2 5}$ & $\mathbf{6 . 0 4 0}$ & $\mathbf{1 7 6 . 0}$ & $\mathbf{4 . 5 2}$ \\
$\mathbf{1 0 . 5}$ & $\mathbf{0 . 6 8 6 2}$ & $\mathbf{0 . 6 7 3 3}$ & $\mathbf{1 . 6 9 7}$ & $\mathbf{7 . 0 6 1}$ & $\mathbf{1 5 3 . 4}$ & $\mathbf{3 . 4 7}$ \\
$\mathbf{1 1 . 5}$ & $\mathbf{0 . 6 0 5 6}$ & $\mathbf{0 . 5 9 6 9}$ & $\mathbf{1 . 5 0 5}$ & $\mathbf{8 . 0 8 3}$ & $\mathbf{1 3 4 . 7}$ & $\mathbf{2 . 7 0}$ \\
$\mathbf{1 2 . 5}$ & $\mathbf{0 . 5 3 2 4}$ & $\mathbf{0 . 5 2 6 6}$ & $\mathbf{1 . 3 2 8}$ & $\mathbf{9 . 1 6 1}$ & $\mathbf{1 1 7 . 7}$ & $\mathbf{2 . 0 9}$ \\
$\mathbf{1 3 . 5}$ & $\mathbf{0 . 4 6 9 7}$ & $\mathbf{0 . 4 6 6 0}$ & $\mathbf{1 . 1 7 5}$ & $\mathbf{1 0 . 1 9 7}$ & $\mathbf{1 0 3 . 1}$ & $\mathbf{1 . 6 2}$ \\
$\mathbf{1 4 . 5}$ & $\mathbf{0 . 4 1 6 0}$ & $\mathbf{0 . 4 1 3 8}$ & $\mathbf{1 . 0 4 3}$ & $\mathbf{1 1 . 1 6 1}$ & $\mathbf{9 0 . 6}$ & $\mathbf{1 . 2 8}$ \\
$\mathbf{1 5 . 5}$ & $\mathbf{0 . 3 6 7 0}$ & $\mathbf{0 . 3 6 5 8}$ & $\mathbf{0 . 9 2 2}$ & $\mathbf{1 2 . 0 9 2}$ & $\mathbf{7 9 . 0}$ & $\mathbf{0 . 9 9}$ \\
$\mathbf{1 6 . 5}$ & $\mathbf{0 . 3 2 5 1}$ & $\mathbf{0 . 3 2 4 6}$ & $\mathbf{0 . 8 1 8}$ & $\mathbf{1 2 . 9 1 1}$ & $\mathbf{6 9 . 0}$ & $\mathbf{0 . 7 8}$ \\
$\mathbf{1 7 . 5}$ & $\mathbf{0 . 2 8 8 4}$ & $\mathbf{0 . 2 8 8 3}$ & $\mathbf{0 . 7 2 7}$ & $\mathbf{1 3 . 6 3 3}$ & $\mathbf{5 9 . 9}$ & $\mathbf{0 . 6 1}$ \\
$\mathbf{1 8 . 5}$ & $\mathbf{0 . 2 5 5 9}$ & $\mathbf{0 . 2 5 6 0}$ & $\mathbf{0 . 6 4 5}$ & $\mathbf{1 4 . 2 6 3}$ & $\mathbf{5 1 . 6}$ & $\mathbf{0 . 4 8}$ \\
$\mathbf{1 9 . 5}$ & $\mathbf{0 . 2 2 7 0}$ & $\mathbf{0 . 2 2 7 4}$ & $\mathbf{0 . 5 7 3}$ & $\mathbf{1 4 . 8 0 2}$ & $\mathbf{4 4 . 1}$ & $\mathbf{0 . 3 8}$ \\
$\mathbf{2 1 . 0}$ & $\mathbf{0 . 1 9 0 3}$ & $\mathbf{0 . 1 9 0 9}$ & $\mathbf{0 . 4 8 1}$ & $\mathbf{1 5 . 4 4 5}$ & $\mathbf{3 4 . 5}$ & $\mathbf{0 . 2 7}$ \\
$\mathbf{2 3 . 0}$ & $\mathbf{0 . 1 5 0 3}$ & $\mathbf{0 . 1 5 1 1}$ & $\mathbf{0 . 3 8 1}$ & $\mathbf{1 6 . 0 6 8}$ & $\mathbf{2 3 . 9}$ & $\mathbf{0 . 1 7}$ \\
$\mathbf{2 5 . 0}$ & $\mathbf{0 . 1 1 8 8}$ & $\mathbf{0 . 1 1 9 7}$ & $\mathbf{0 . 3 0 2}$ & $\mathbf{1 6 . 4 8 4}$ & $\mathbf{1 6 . 2}$ & $\mathbf{0 . 1 0}$ \\
$\mathbf{2 7 . 0}$ & $\mathbf{0 . 0 9 4 0}$ & $\mathbf{0 . 0 9 5 0}$ & $\mathbf{0 . 2 4 0}$ & $\mathbf{1 6 . 7 5 4}$ & $\mathbf{1 0 . 6}$ & $\mathbf{0 . 0 7}$ \\
$\mathbf{3 0 . 0}$ & $\mathbf{0 . 0 6 6 1}$ & $\mathbf{0 . 0 6 7 3}$ & $\mathbf{0 . 1 7 0}$ & $\mathbf{1 6 . 9 9 0}$ & $\mathbf{5 . 5}$ & $\mathbf{0 . 0 3}$ \\
35.0 & 0.0368 & 0.0379 & 0.096 & 17.156 & 1.8 & 0.01 \\
40.0 & 0.0204 & 0.0213 & 0.054 & 17.209 & 0.6 & 0.00 \\
& & & & & & \\
\hline
\end{tabular}

1) The measured gaps are shown in bold italic. Intermediate gap values were obtained by linear interpolation of log of field vs. gap.

2) Fields were not measured for gaps larger than $30.0 \mathrm{~mm}$. For gaps beyond $30.0 \mathrm{~mm}$, the dependency on the gap was extrapolated from the field at the two largest measured gaps $(27.0 \mathrm{~mm}$ and $30.0 \mathrm{~mm})$.

3,4) See footnote for Table 2 for definitions.

5) Zero-emittance calculation using $K_{\text {eff }}$ for on-axis radiation $(\theta=0)$ for beam energy 7.0 GeV. See footnote for Table 2 for additional information.

6) Zero-emittance calculation at beam energy 7.0 Gev and current $100 \mathrm{~mA}$, using $B_{\text {peak }}$ and the full number of undulator periods $(N=88)$. 


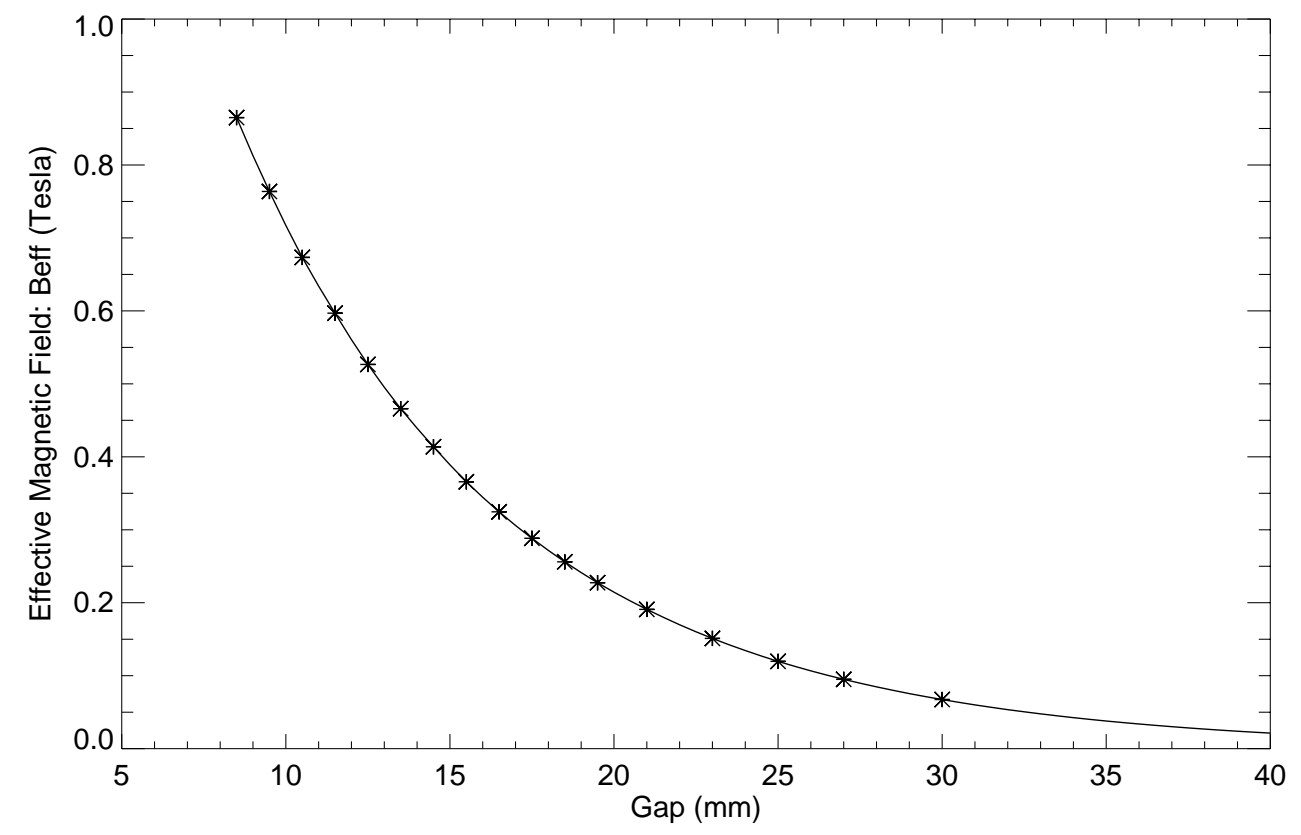

Figure C1. Measured effective magnetic field $B_{\text {eff }}$ as a function of gap for one $2.7-\mathrm{cm}$ period device (minimum gap is $8.5 \mathrm{~mm}$ ). The data are from Table $\mathrm{C} 1$. The solid line is the interpolation/extrapolation of field vs. gap.

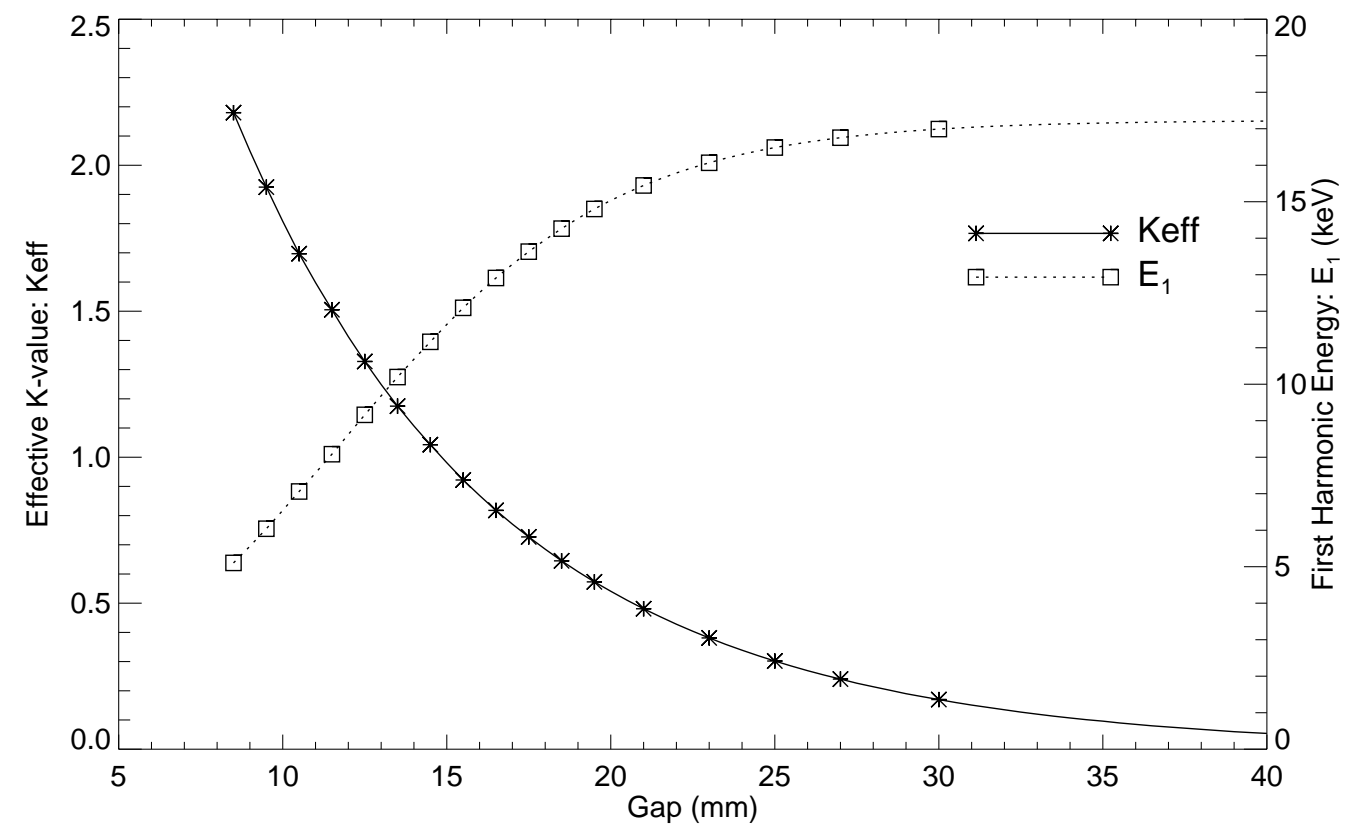

Figure C2. Measured effective $K$ value and calculated first harmonic energy $\left(E_{l}\right)$ for onaxis radiation for $7.0 \mathrm{GeV}$ beam energy as a function of gap. The data are from Table $\mathrm{C} 1$. The solid line is the interpolation/extrapolation of $K$ vs. gap and the dotted line is calculated from the interpolated/extrapolated values. The minimum gap is $8.5 \mathrm{~mm}$ $\left(K_{\text {eff }}=2.180\right)$ and minimum calculated energy is $5.10 \mathrm{keV}$. The useful tuning range for the first harmonic is approximately $5-15 \mathrm{keV}$. 


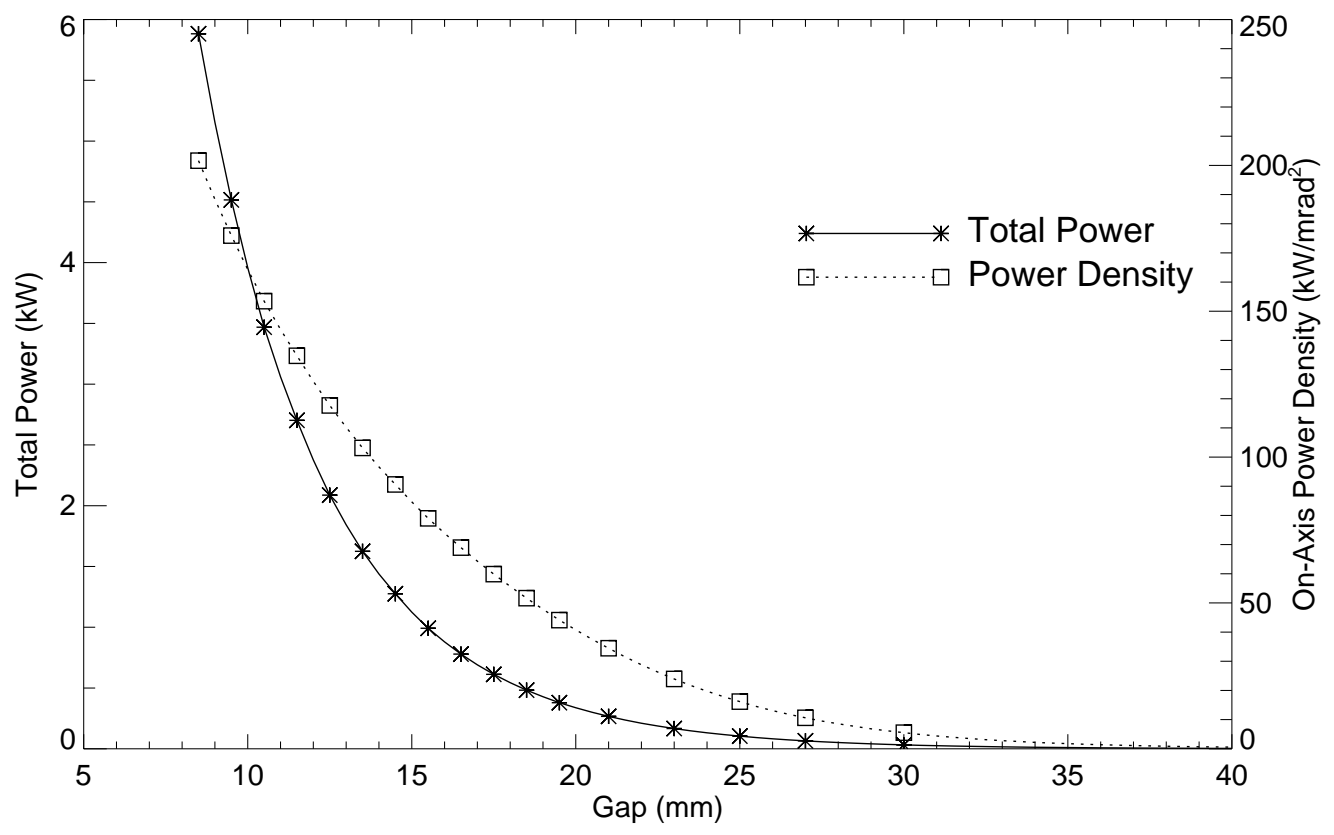

Figure C3: Total power and on-axis power density for the $2.7-\mathrm{cm}$ device (zero-emittance calculation) vs. gap for a beam energy of $7.0 \mathrm{GeV}$ and $100 \mathrm{~mA}$ current. The data are from Table C1.

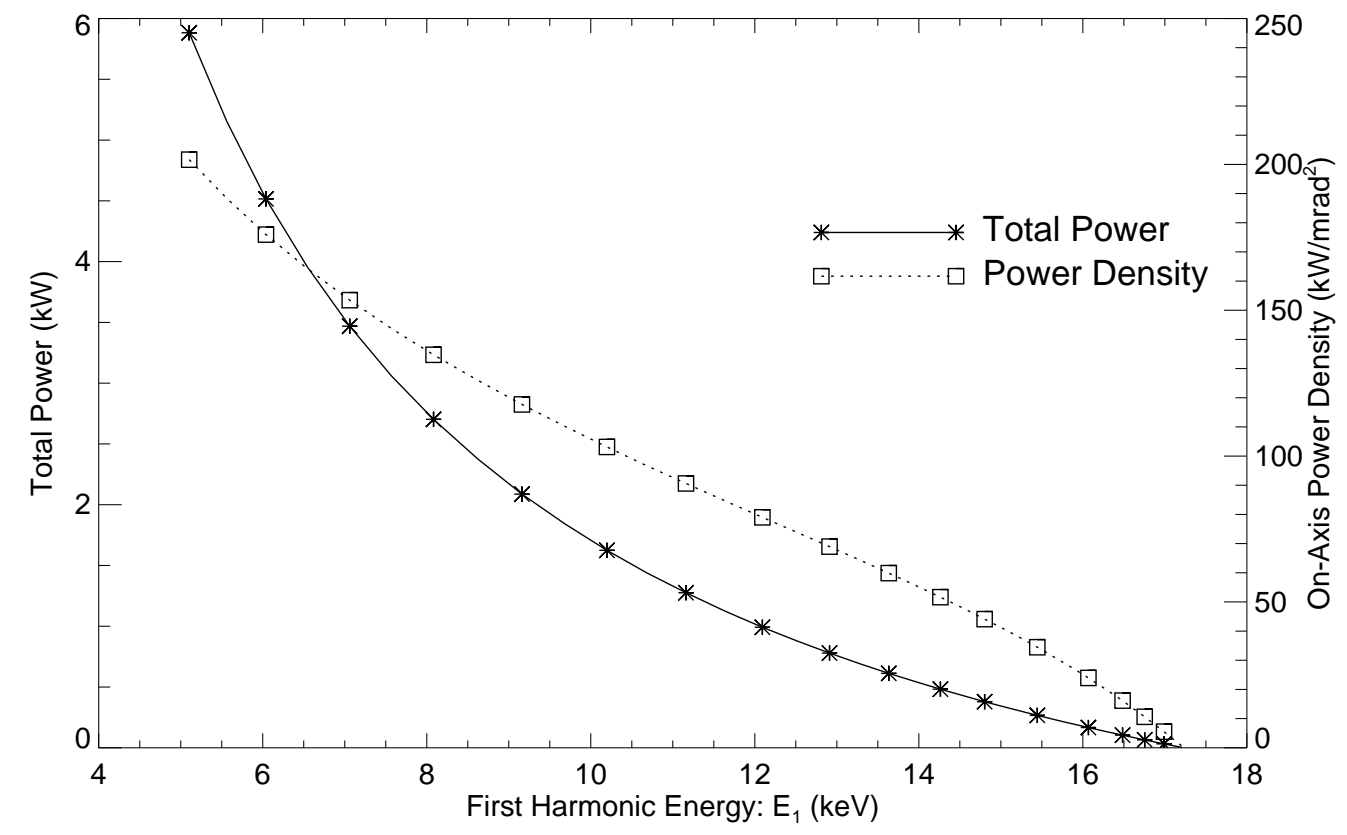

Figure C4: Total power and on-axis power density for the 2.7-cm device (zero-emittance calculation) vs. first harmonic energy for a beam energy of $7.0 \mathrm{GeV}$ and $100 \mathrm{~mA}$ current. The data are from Table C1. 


\section{Appendix D: On-Axis Brilliance for APS Bending Magnet, Wiggler, and Other IDs}

For completeness, we compare the calculated on-axis brilliance for all APS planar devices $(L=2.4 \mathrm{~m})$ vs. the bending magnet and the wiggler for the present low-emittance lattice (Figure D1). Here we have calculated the brilliances up to $100 \mathrm{keV}$, which includes many harmonics (harmonic 33 for Undulator A and harmonic 19 for the 2.7-cmperiod device). The magnetic field errors were not taken into account, and the brilliance drop at $100 \mathrm{keV}$ may be estimated to about a factor of two from what is shown.

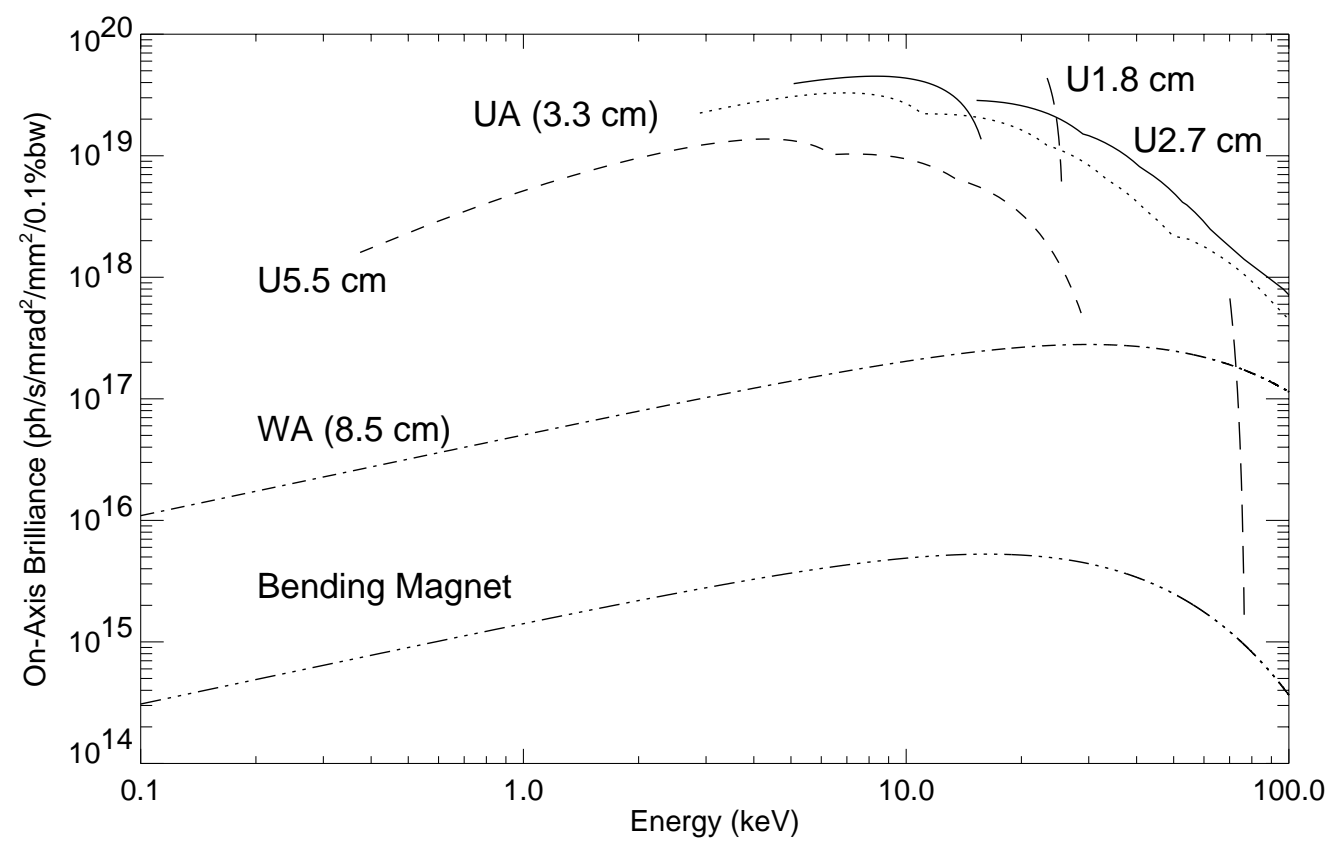

Figure D1. Comparison of the on-axis brilliance for the APS bending magnet, wiggler, and "other IDs" vs. Undulator A (3.3 cm period; dotted curve) for the low-emittance lattice. The bending magnet, with a critical energy $E_{c}$ of $19.5 \mathrm{keV}$, is shown as the dotted-dotted-dashed curve. The wiggler, Wiggler A (WA), with an $8.5-\mathrm{cm}$ period, is shown as the dotted-dashed curve. The wiggler was set at $18.1 \mathrm{~mm}$ gap (peak magnetic field $B_{\text {peak }}=1.1 \mathrm{~T}, E_{c}=35.9 \mathrm{keV}$ ). The undulators cover particular energy ranges as is easily seen. The minimum gap is $10.5 \mathrm{~mm}$ except for the $2.7-\mathrm{cm}$ device which is 8.5 $\mathrm{mm}$. The 5.5-cm-period device is used for experiments at lower energies and only the first, third, and fifth harmonics are shown (U5.5 cm, dashed curve). The 1.8-cm-period device (U1.8 cm, long-dash) is a special device and the first and third harmonics are shown. Note the very limited tuning range for this device. This is because the $K$ value is small even at closed gap (0.45 at $10.5 \mathrm{~mm}$ gap) and the intensity drops quickly for small changes in the $K$ value (undulator gap). As can be seen, the 2.7-cm-period device (U2.7 $\mathrm{cm}$, solid curve) gives a higher brilliance than does Undulator A over the entire energy range where they overlap with only a modest loss in brilliance between the first and third harmonic. Higher harmonics were included in the calculations for these devices (harmonic 33 for Undulator A and harmonic 19 for the 2.7-cm-period device). 


\section{References}

[1] B. Lai, A. Khounsary, R. Savoy, L. Moog, and E. Gluskin, Argonne National Laboratory Report, ANL/APS/TB-3, February 1993.

[2] Roger J. Dejus, Barry Lai, Elizabeth R. Moog, and Efim Gluskin, Argonne National Laboratory Report, ANL/APS/TB-17, May 1994.

[3] M. Sanchez del Rio and R.J. Dejus, SPIE Proc. 3152, 148 (1997).

[4] R.J. Dejus and A. Luccio, Nucl. Instrum. Methods, A347, 61 (1994).

[5] D. Haeffner, Argonne National Laboratory. Private communication, 2002. First beam was delivered on August 9, 1995 at 20:05.

[6] Z. Cai, R.J. Dejus, P. Den Hartog, Y. Feng, E. Gluskin, D. Haeffner, P. Ilinski, B. Lai, D. Legnini, E.R. Moog, S. Shastri, E. Trakhtenberg, I. Vasserman, and W. Yun, "APS Undulator Radiation - First Results", Rev. Sci. Instrum. 67, CD-ROM (1996).

[7] P. Ilinski, R.J. Dejus, E. Gluskin, and T.I. Morrison, SPIE Proc. 2856, 16 (1996).

[8] S.D. Shastri, R.J. Dejus, and D.R. Haeffner, J. Synchrotron Rad. 5, 67 (1998).

[9] P. Ilinski, Argonne National Laboratory Report, ANL/APS/TB-33, January 1998.

[10] I. Vasserman, Argonne National Laboratory Report, LS-253, January 1996.

[11] B. Diviacco and R.P. Walker, Nucl. Instrum. Methods, A368, 522 (1996).

[12] K.-J. Kim, Nucl. Instrum. Methods, A246, 67 (1986).

[13] K.-J. Kim, AIP Proceedings 184, Physics of Particle Accelerators, vol. 1, p. 565 (1989).

[14] G.K. Shenoy, Argonne National Laboratory Report, ANL/APS/TB-43, April 2002.

[15] H. Wiedemann, Particle Accelerator Physics, (Springer-Verlag, 1993).

[16] L. Emery, "Recent Operational Data on Continuous Top-Up Operation at The Advanced Photon Source," 2001 U.S. Particle Accelerator Conference, p. 2599 (2001).

[17] L. Emery, M. Borland, R. Dejus, E. Gluskin, and E. Moog., "Progress and Prospects Toward Brightness Improvements at the Advanced Photon Source," 2001 U.S. Particle Accelerator Conference, p. 2602 (2001).

[18] L. Emery, Argonne National Laboratory. Private communication, 2002.

[19] G.K. Shenoy, P.J. Viccaro, and D.M. Mills, Argonne National Laboratory Report, ANL-88-9, p. 40, February 1988.

[20] S.C. Gottschalk, K.E. Robinson, D.C. Quimby, K.W. Kangas, I. Vasserman, R. Dejus, and E. Moog, "Multipole and Phase Tuning Methods for Insertion Devices," Rev. Sci. Instrum. 67 CD-ROM (1996).

[21] I.B. Vasserman, N.A. Vinokurov, and R.J. Dejus, "Phasing of the Insertion Devices at the APS FEL Project," SRI99, Stanford, CA, AIP Proceedings 521, p. 368 (2000). 